\title{
OPTIMIZING CONTROL OF SHELL ECO-MARATHON PROTOTYPE VEHICLE TO MINIMIZE FUEL CONSUMPTION
}

\author{
A Thesis \\ presented to \\ the Faculty of California Polytechnic State University, \\ San Luis Obispo
}

\author{
In Partial Fulfillment \\ of the Requirements for the Degree \\ Master of Science in Electrical Engineering
}

by

Chad Louis Bickel

April 2017 
(C) 2017

Chad Louis Bickel

ALL RIGHTS RESERVED 


\section{COMMITTEE MEMBERSHIP}

TITLE: Optimizing Control of Shell Eco-marathon

Prototype Vehicle to Minimize Fuel Consumption

AUTHOR: Chad Louis Bickel

DATE SUBMITTED: April 2017

COMMITTEE CHAIR: Art MacCarley, Ph.D., PE.

Professor of Electrical Engineering

COMMITTEE MEMBER: Joseph Mello, Ph.D.

Professor of Mechanical Engineering

COMMITTEE MEMBER: Markus Krug, Ph.D.

Lecturer of Electrical Engineering 


\begin{abstract}
Optimizing Control of Shell Eco-marathon Prototype Vehicle to Minimize Fuel Consumption
\end{abstract}

Chad Louis Bickel

Every year the automotive industry strives to increase fuel efficiency in vehicles. When most vehicles are designed, fuel efficiency cannot always come first. The Shell Eco-marathon changes that by challenging students everywhere to develop the most fuel-efficient vehicle possible. There are many different factors that affect fuel efficiency, and different teams focus on different vehicle parameters. Currently, there is no straightforward design tool that can be used to help in Shell Eco-marathon vehicle design. For this reason, it is difficult to optimize every vehicle parameter for maximum fuel efficiency.

In this study, a simulation is developed by using basic vehicle models and experimental data to accurately represent any prototype-class vehicle in the Shell Ecomarathon. This simulation is verified using different experimental data from an on-vehicle data acquisition system. An easy-to-use design tool is developed, and this tool is used to optimize driving strategy and final drive ratio to maximize fuel efficiency. 


\section{ACKNOWLEDGMENTS}

There are many people that helped me in the process of developing and writing this thesis. Thank you Art MacCarley for guiding me throughout the entire process; you always motivated me to do more and challenged me to do everything better. Thank you Dr. Mello for inspiring me to develop a simulation of the Supermileage vehicle, and thank you for supporting me and the team in general. Thank you Dr. Krug for bringing a different perspective to the table and encouraging me to rethink my methodologies.

Thanks to the Electrical Engineering faculty for teaching me all the skills I utilized in this study, and thanks to the Mechanical Engineering department for funding the Supermileage team.

Thanks to all the Cal Poly Supermileage presidents that supported this endeavor: Sean Michel, Dorian Capps, and Lucas Rybarczyk. Thanks to everyone else on the team for helping me out too!

Lastly, I'd like to thank all my friends and family who motivated and supported me throughout this process. I never imagined doing all this, but here I am thanks to all of you. 
TABLE OF CONTENTS

Page

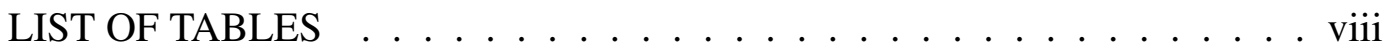

LIST OF FIGURES . . . . . . . . . . . . . . . . . ix

\section{CHAPTER}

1 Introduction $\ldots \ldots \ldots \ldots \ldots \ldots \ldots$

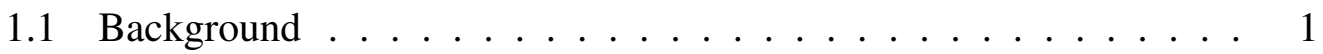

1.2 Previous Studies . . . . . . . . . . . . . . 2

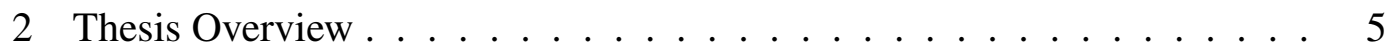

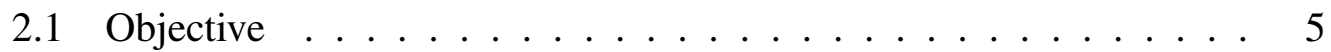

2.2 Method ........................ 5

3 Developing the Vehicle Model . . . . . . . . . . . . . . . 6

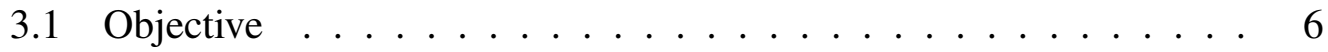

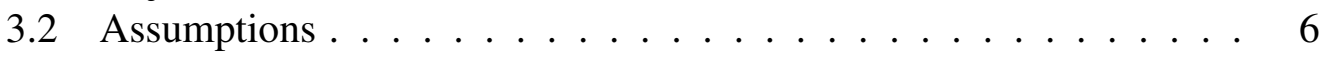

3.3 Key Equations . . . . . . . . . . . . . . . . 7

3.3 .1 Vehicle Forces . . . . . . . . . . . . 7

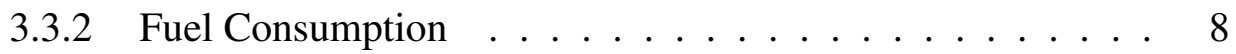

3.3.3 Starting Model .................... 9

3.3.4 Clutch Not Fully Engaged . . . . . . . . . . . . . . 10

3.3.5 Clutch Fully Engaged . . . . . . . . . . . . . . . 11

3.4 State-Space Model . . . . . . . . . . . . . . . . . . 12

3.5 Simulation Development . . . . . . . . . . . 13

4 Analyzing Data to Build and Validate Model . . . . . . . . . . . . . . 24

4.1 Data Acquisition System . . . . . . . . . . . . . . . . 25

4.2 Road Load Coefficients . . . . . . . . . . . . . . . . . . . . . . . 28

4.3 The Affect of Weight on Road Load . . . . . . . . . . . . . . . . . . . 37

4.4 Engine Torque and BSFC Curves . . . . . . . . . . . . . . . . . 40

4.5 Drivetrain Efficiency . . . . . . . . . . . . . . . 41

4.6 Clutch Torque Curve . . . . . . . . . . . . . . . . . . . 46

4.7 Starting Penalties . . . . . . . . . . . . . . . 47 
4.8 Verifying the Simulation ............... 51

5 Vehicle Parameter Analysis . . . . . . . . . . . . . . . . 57

5.1 User Interface . . . . . . . . . . . . . . . . . . . . 57

5.2 Vehicle Weight Analysis . . . . . . . . . . . . . . 59

5.3 Optimizing Vehicle Speed and Gear Ratio . . . . . . . . . . . . . 60

5.4 Optimization Discussion . . . . . . . . . . . . . . 67

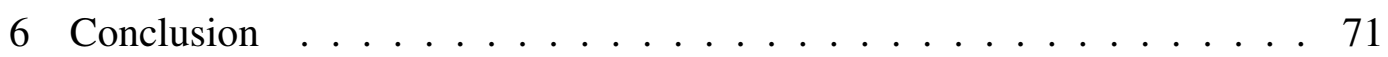

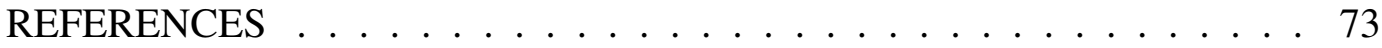

\section{APPENDICES}

A. User Interface Instructions . . . . . . . . . . . . . . . 76

B. MATLAB Code . . . . . . . . . . . . . . . . 78 


\section{LIST OF TABLES}

Table

Page

1 Optimization Results . . . . . . . . . . . . . . . . 62

2 Optimization Comparison . . . . . . . . . . . . 67 


\section{LIST OF FIGURES}

Figure

Page

1 Cal Poly Supermileage 2016 Vehicle . . . . . . . . . . . . . . . 1

2 Top-Level View of Simulink Model . . . . . . . . . . . . . . . . . . . 14

3 Plant Subsystem of Simulink Model . . . . . . . . . . . . . . . . 15

4 Lap Calculation Subsystem . . . . . . . . . . . . . . . . . . . 15

5 State Model Subsystem . . . . . . . . . . . . . . . . . . . 17

6 Road Load Forces Subsystem . . . . . . . . . . . . . . . . . . . . . 18

7 Fuel Consumption Model Subsystem . . . . . . . . . . . . . . . . . 19

8 Torque Curves and Starting Model Subsystem . . . . . . . . . . . 21

9 Clutch Curve Subsystem . . . . . . . . . . . . . . . 22

10 Clutch Locked Determination Subsystem . . . . . . . . . . . . . 23

11 RaceCapture in Vehicle . . . . . . . . . . . . . . . . . 25

12 Speed Data Gathered from RaceCapture . . . . . . . . . . . . . . 27

13 Engine Speed and Pulse Width Data Gathered from RaceCapture . . 28

14 Logged Speed versus Lap Position . . . . . . . . . . . . . . . . 31

15 Altitude versus Lap Position . . . . . . . . . . . . . . . . . 32

16 Elevation Profile of Test Track . . . . . . . . . . . . . 33

17 Coastdown Velocity Data from 0.5 to 0.9 Lap Position . . . . . . . . 35

18 Road Load versus Vehicle Speed . . . . . . . . . . . . . . . . . 36

$19 F_{r r}$ versus Total Vehicle Weight $\ldots . . . . . . . . . .39$

20 Fuel Flow Rate versus Engine Speed . . . . . . . . . . . . . . . 41

21 Engine Speed versus Time . . . . . . . . . . . . . . 42

22 Clutch Housing Speed versus Time . . . . . . . . . . . . . . . . 43

23 Vehicle Acceleration versus Engine Speed . . . . . . . . . . . . . . . 44

24 Drivetrain Efficiency versus Engine Speed . . . . . . . . . . . . . . 45

25 Clutch Torque versus Engine Speed . . . . . . . . . . . . . . . 47

26 Total Fuel Consumption versus Time . . . . . . . . . . . . . . . 48

27 Engine Coolant Temperature versus Time . . . . . . . . . . . . . . 49

28 Time Taken to Start Motor . . . . . . . . . . . . . . . 50

29 Fuel Consumed per Start . . . . . . . . . . . . . . . 51

30 Vehicle Speed versus Time . . . . . . . . . . . . . . 52

31 Zoomed In Vehicle Speed versus Time . . . . . . . . . . . . 53

32 Engine Speed versus Time . . . . . . . . . . . . . . 54

33 Zoomed In Engine Speed versus Time . . . . . . . . . . . . . . 55

34 Total Fuel Consumption versus Distance . . . . . . . . . . . . . 56

35 Simulation User Interface . . . . . . . . . . . . . . . . . . 58

36 Fuel Efficiency versus Weight . . . . . . . . . . . . . 59 
37 Fuel Efficiency versus Gear Ratio, Varying Vehicle Speed . . . . . . 63

38 Fuel Efficiency versus Gear Ratio, Constant $v_{\min }$ and $v_{\max } \ldots \ldots 4$

39 Fuel Efficiency versus Maximum Speed, Constant $\eta$. . . . . . . . . . 65

40 Fuel Efficiency versus Minimum Speed, Constant $\eta$. . . . . . . . . 66

41 Fuel Efficiency versus Maximum and Minimum Speed, Constant $\eta$. 67

42 Engine Speed versus Time . . . . . . . . . . . . . . . . 68

43 Vehicle Speed versus Time . . . . . . . . . . . . . . . . 69

44 Fuel Consumption versus Distance . . . . . . . . . . . . . . 70 


\section{NOMENCLATURE}

$\dot{m}_{i} \quad$ Fuel Injector Flow Rate, $m L /$ fuel $m s$

$\eta \quad$ Final Drive Ratio

$\gamma \quad$ Drivetrain Efficiency

$\omega^{\prime} \quad$ Clutch Shoe Engagement Speed, $\mathrm{rad} / \mathrm{s}$ or $\mathrm{rpm}$

$\omega_{e} \quad$ Engine Speed, $\mathrm{rad} / \mathrm{s}$ or $\mathrm{rpm}$

$\omega_{i} \quad$ Engine Idle Speed, $\mathrm{rad} / \mathrm{s}$ or $\mathrm{rpm}$

$\tau_{c} \quad$ Torque from Clutch, $l b f f t$

$\tau_{e} \quad$ Torque produced by Engine, $l b f f t$

$\theta \quad$ Angle of Road, ${ }^{\circ}$ or $\mathrm{rad}$

$\underline{t}_{R C} \quad$ Time Vector Data, $s$

$\underline{v}_{R C} \quad$ Speed Vector Data, $f t / s$

$\underline{x} \quad$ State Variable

$B S F C\left(\omega_{e}\right)$ Brake Specific Fuel Consumption Table, $(s l u g / s) /(l b f f t / s)$

$C_{c} \quad$ Clutch Engagement Coefficient, $(l b f f t) /\left(\mathrm{rad}^{2} / \mathrm{s}^{2}\right)$

$C_{D} \quad$ Experimental Coefficient of Aerodynamic Drag, $l b f /\left(f t^{2} / s^{2}\right)$

$C_{V} \quad$ Experimental Coefficient of Wheel and Bearing Resistance, $l b f /(f t / s)$

$F_{d t} \quad$ Force Exerted by Drivetrain on Vehicle, $l b f$

$f_{P W} \quad$ Fuel Injector Pulse Width, fuel ms

$F_{r l} \quad$ Road Load Force on Vehicle, $l b f$

$F_{r r} \quad$ Experimental Constant of Rolling Resistance, $l b f$

$f_{r r} \quad$ Coefficient of Rolling Resistance

$g \quad$ Acceleration Due to Gravity, $\mathrm{ft} / \mathrm{s}^{2}$

$J_{e} \quad$ Engine Mass Moment of Inertia, slugft ${ }^{2}$ 


\author{
$K_{\omega} \quad$ Starting Engine Acceleration Constant, $\mathrm{rad} / \mathrm{s}^{2}$ \\ $K_{m_{f}} \quad$ Starting Fuel Consumption Rate Constant, slug/s \\ $m_{f} \quad$ Total Mass of Fuel Consumed, slug \\ $m_{v} \quad$ Total Vehicle Mass, slug \\ $p \quad$ Vehicle Position, $f t$ \\ poly $(x, y)$ Polynomial Regression Function \\ $r \quad$ Rear Wheel Radius, $f t$ \\ $u \quad$ Driver Input to Engine \\ $v \quad$ Vehicle Speed, $f t / s$ \\ $v_{\max }$ Maximum Vehicle Speed, $f t / s$ \\ $v_{\min }$ Minimum Vehicle Speed, $f t / s$
}




\section{Introduction}

This section introduces the problem and gives background information on previous studies in the field of high-efficiency vehicles.

\subsection{Background}

The Cal Poly Supermileage club designs and builds an extremely efficient vehicle every year. This vehicle is powered by a small internal combustion engine, and it has enough room for one person to drive lying down. Figure 1 shows the 2016 version of the prototype vehicle.

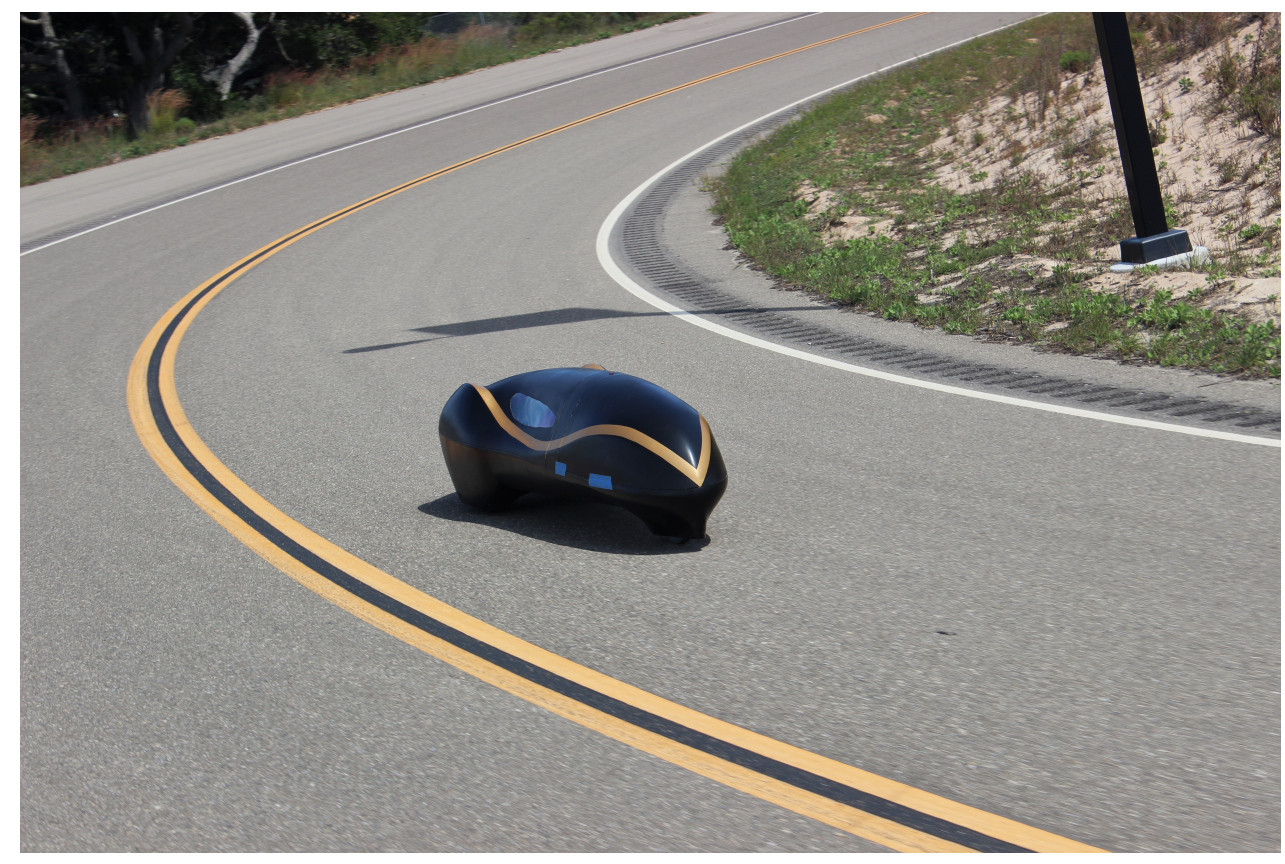

Figure 1: Cal Poly Supermileage 2016 Vehicle

This vehicle competes in the Shell Eco-marathon, in which vehicles must travel 6 miles within 24 minutes using the least amount of fuel possible[17]. The track is 
composed of portions of closed-off city streets, which are often uneven and rough. Most drivers in the competition use the "burn and coast" method. This means that a vehicle accelerates until a certain speed has been reached, and then the engine is cut off. The vehicle then coasts until a minimum speed is reached, and the engine is started again. This ensures that fuel is only consumed when acceleration is needed. When the engine is running, the throttle is set at $100 \%$, so the driver only controls whether the engine is running or not.

Designing a new vehicle is challenging; there are many important aspects of the vehicle to consider. These include weight, engine size and power, and aerodynamic efficiency. Historically, the team has sought to minimize weight and road load forces; a small engine has also been used for maximum efficiency. There are often tradeoffs for these parameters. For example, an aerodynamic shape often results in poor driver visibility, which could affect the driver's abilities. The importance of each design parameter has mostly been unknown in the past, resulting in a sub-optimal vehicle. Additionally, the driver does not know what speeds are most efficient to drive at.

\subsection{Previous Studies}

There have been many previous studies looking at optimizing fuel efficiency in consumer vehicles. Many deal with the design of fuel-efficient vehicles or the determination of optimal highway speeds. However, many of these are not useful when applied to Shell Eco-marathon prototypes, which do not follow traditional vehicle designs or travel at high speeds. One applicable study looked at optimizing vehicle speed and final drive ratio to minimize fuel consumption at low speeds, and 
they found that $37 \%$ of fuel could be saved with good driving technique and proper gear selection[15]. Therefore, driving strategy should have a significant affect on fuel efficiency for low-speed vehicles.

A very thorough study of a prototype Shell Eco-marathon vehicle was done by the team of Pac-car II. It was one of the most efficient vehicles ever made for the Shell Eco-marathon[16]. Many different aspects of prototype vehicles were analyzed and optimized for fuel efficiency in the study. However, Pac-car II was powered by a hydrogen fuel cell, so many of the results may not be valid for a gasolinepowered vehicle. The most important study in the Pac-car II report was optimizing driving strategy. This study was completed by using a first-order approximation of the theoretical system and then optimizing it using linear programming. In the end, a fairly complicated driving strategy was developed that is difficult for a driver to be able to memorize. Additionally, the complex driving strategy may not be truly optimal due to the approximated system.

A similar study was conducted in which the driver is assumed to be a perfect bang-bang controller[6]. A nonlinear programming method was used to find the optimal controller speeds. The resulting optimal control law is much easier for an actual driver to follow. For this reason, the simulation developed in this study will assume the driver will only need a minimum and maximum vehicle speed, acting like a bang-bang controller. This will simplify the optimization so that no system linearization is required. Additionally, for this study, the final drive ratio will be optimized at the same time as vehicle speeds, which has not been done in previous Shell Eco-marathon studies.

Other studies have been conducted to find the optimal speeds of prototype elec- 
tric vehicles[8] and prototype fuel-cell vehicles[13], however these systems are quite different than gasoline-powered vehicles.

Various studies have also made detailed simulations of Shell Eco-marathon vehicles[2][12]. Most of these simulations are either difficult to use or slow. The simulation developed in this study will be much faster and easier to use, making a more practical design tool.

The most closely related study was done by Eric Griess at Cal Poly in 2015[5]. A simulation was developed of the Cal Poly Supermileage vehicle with a focus on engine and powertrain optimization. This simulation had a few uncertainties, including unknown drag coefficient, drivetrain efficiency, and clutch characteristics. Ultimately, a tool to optimize engine torque and BSFC curves was developed, but this does not help in designing other vehicle parameters. However, the engine data gathered will be used in a later part of this study(section 4.4). 


\section{Thesis Overview}

This section presents the scope of this study.

\subsection{Objective}

The objective of this project is to develop and verify a simulation that can be used to quickly determine the relative importance of major vehicle parameters and optimize the speed and final drive ratio of the vehicle.

\subsection{Method}

This project will be completed in the following order:

1. Develop a simulation using MATLAB/Simulink

2. Gather real vehicle data using an on-board data acquisition system

3. Analyze data and determine vehicle parameters for simulation

4. Compare simulation outputs to actual vehicle outputs

5. Develop a simple user interface for simulation to allow for quick trade studies

6. Optimize speed and final drive ratio 
3 Developing the Vehicle Model

In the following sections, the equations to accurately model the prototype vehicle are developed.

\subsection{Objective}

The objective of this portion of the project is to develop a model that accurately represents the dynamics of the vehicle and can still be simulated within a few seconds.

\subsection{Assumptions}

- The track has no turns, so only one positional variable is needed

- The engine intake and coolant temperatures are constant, so thermodynamics can be neglected

- The vehicle weight is constant

- No fluctuating air conditions (wind, temperature, density)

- Perfect driver (no distractions, obstactles, or other vehicles on track)

- Road load fits a second order polynomial

- Drivetrain and wheel inertia is much smaller than the inertia of the entire vehicle

- Engine acceleration and fuel consumption rate are constant before idle speed is reached 


\subsection{Key Equations}

The following equations[4] can be used to represent a vehicle under the above assumptions:

\subsubsection{Vehicle Forces}

Equation 1 shows the sum of the forces on the vehicle is equal to the force from the drivetrain minus the force from road load:

$$
\sum F_{v}=F_{d t}-F_{r l}
$$

The road load force is a function of vehicle speed and road slope[4], shown below:

$$
F_{r l}(v, \theta)=C_{D} v^{2}+C_{V} v+F_{r r}+m_{v} g \sin (\theta)
$$

Where:

$m_{v}$ is the total mass of the vehicle $(\operatorname{slug})$

$g$ is the acceleration due to gravity $\left(\mathrm{ft} / \mathrm{s}^{2}\right)$

$\theta$ is the angle of the uneven road (deg or $r a d$ )

$F_{r l}$ always opposes the direction of motion of the vehicle. The $v^{2}$ term is caused by aerodynamic drag; the $v$ term is primarily caused by bearing rolling resistance and some tire effects; the constant term $F_{r r}$ is caused by traditional rolling resistance from the tires. The last term is caused by the slope of the road. Note that $C_{D}$ is not the traditional coefficient of drag. $C_{D}$ in this case includes the frontal area, 
drag coefficient, and air density combined into one number in units of $l b f /\left(f t^{2} / s^{2}\right)$.

Equation 3 shows the relationship between force on the rear wheel and torque from the clutch.

$$
F_{d t}=\frac{\tau_{c}\left(\omega_{e}, v\right) \eta \gamma}{r}
$$

Where:

$\omega_{e}$ is the engine speed $(\mathrm{rad} / \mathrm{s}$ or $\mathrm{rpm})$

$\tau_{c}$ is the torque from the clutch $(l b f f t)$

$\gamma$ is the drivetrain efficiency ( 0 to 1 )

$\eta$ is the final drive ratio

$r$ is the rear wheel radius $(f t)$

Equation 4 shows the relationship between speed and total vehicle force:

$$
\dot{v}(t)=\frac{F_{v}}{m}
$$

Equation 5 indicates the relationship between vehicle position and speed:

$$
\dot{p}(t)=v(t)
$$

\subsubsection{Fuel Consumption}

Equation 6 shows how fuel consumption rate relates to engine power: 


$$
\dot{m}_{f}(t)=\omega_{e} \tau_{e}\left(\omega_{e}\right) B S F C\left(\omega_{e}\right)
$$

Where:

$m_{f}$ is the total mass of fuel consumed (slug)

$\tau_{e}$ is the torque produced by the engine $(l b f f t)$

$\operatorname{BSFC}\left(\omega_{e}\right)$ is the Brake Specific Fuel Consumption Table $((\mathrm{slug} / \mathrm{s}) /(\mathrm{lbfft} / \mathrm{s}))$

When the engine is running, there are three cases to consider:

1. The engine speed is below idle

2. The centrifugal clutch is not fully engaged

3. The centrifugal clutch is fully engaged

\subsubsection{Starting Model}

When the driver first wishes to start the engine, it takes a small amount of time and fuel to reach idle speed. This could be modeled using an electric starter motor model, however this adds significant complexity to the system without adding much accuracy. For this reason, a constant engine acceleration and fuel consumption rate is assumed.

When $\omega_{e}<\omega_{i}$, Equations 7 and 8 are true.

$$
\dot{\omega}_{e}=K_{\omega}
$$




$$
\dot{m}_{f}=K_{m_{f}}
$$

Where:

$\omega_{i}$ is the engine idle speed $(\mathrm{rad} / \mathrm{s})$

$K_{\omega}$ is the starting engine acceleration constant $\left(\mathrm{rad} / \mathrm{s}^{2}\right)$

$K_{m_{f}}$ is the starting fuel consumption rate constant $(\mathrm{slug} / \mathrm{s})$

\subsubsection{Clutch Not Fully Engaged}

For case 2, the engine and vehicle can be considered as 2 separate rigid bodies.

$$
J_{e} \dot{\omega}_{e}=\sum M_{e}=\tau_{e}\left(\omega_{e}\right)-\tau_{c}\left(\omega_{e}, v\right)
$$

Where:

$J_{e}$ is the mass moment of inertia of the engine $\left(\operatorname{slug} f t^{2}\right)$

$\sum M_{e}$ is the sum of the moments acting on the engine (lbfft)

Equation 10 relates centrifugal clutch torque to engine speed[14]:

$$
\tau_{c}\left(\omega_{e}, v\right)=\left\{\begin{array}{lc}
C_{c}\left(\omega_{e}-\omega^{\prime}\right)^{2} & \omega_{e} \geq \omega^{\prime} \\
0 & \omega_{e}<\omega^{\prime}
\end{array}\right\}
$$

Where:

$C_{c}$ is the clutch engagement coefficient $\left((l b f f t) /\left(\operatorname{rad}^{2} / s^{2}\right)\right)$

$\omega^{\prime}$ is the engine speed at which the clutch shoe moves forward ( $\mathrm{rad} / \mathrm{s}$ or $\left.\mathrm{rpm}\right)$ 
During the conditions of a typical competition run, the engine speed is always greater than the clutch housing speed while the clutch is slipping. When the clutch housing speed reaches the speed of the engine, the dynamic friction between the shoe and the housing becomes static, and the clutch becomes locked. So case 2 is only true when equation 11 is true:

$$
\omega_{e}>\frac{v \eta}{r}
$$

The second term in equation 11 is equal to the speed of the clutch housing. This is linked to the rear wheel through the drivetrain.

\subsubsection{Clutch Fully Engaged}

When equation 11 no longer holds, the clutch is fully engaged. The engine and vehicle are now coupled rigid bodies, so the engine speed and vehicle speed are coupled by the following equation:

$$
\omega_{e}=\frac{v \eta}{r}
$$

Since the clutch is fully engaged, the torque on the drivetrain is exactly the torque produced by the engine:

$$
\tau_{c}\left(\omega_{e}, v\right)=\tau_{e}\left(\omega_{e}\right)
$$




\subsection{State-Space Model}

Before developing a Simulink model, it is useful to show the previous equations in state-space representation. There are 4 state variables in the system:

$$
\underline{x}=\left[\begin{array}{c}
p \\
v \\
\omega_{e} \\
m_{f}
\end{array}\right]
$$

The system output vector is equal to the 4 state variables. There is 1 scalar input to the system, $u$. Since the driver can only control if the engine is running or not (not the specific throttle \%), $u$ is binary: 0 or 1 . When $u$ is 0 , the engine is off, and the vehicle is coasting. When $u$ is 1 , the engine is running, and the vehicle is accelerating at full throttle. Combining the input $u$ and equations 5, 4, 9, and 6 produce the following state equation:

$$
\underline{\dot{x}}=\left[\begin{array}{c}
\dot{p} \\
\dot{v} \\
\dot{\omega}_{e} \\
\dot{m}_{f}
\end{array}\right]=\left[\begin{array}{c}
v \\
\left(\tau_{c}\left(\omega_{e}, v\right) \eta \gamma u / r-\left(C_{D} v^{2}+C_{V} v+F_{r r}\right)\right) / m_{v} \\
\left(\tau_{e}\left(\omega_{e}\right)-\tau_{c}\left(\omega_{e}, v\right)\right) u / J_{e} \\
\omega_{e} \tau_{e}\left(\omega_{e}\right) B S F C\left(\omega_{e}\right) u
\end{array}\right]
$$

However, notice that equation 15 is only valid while the clutch is not fully engaged. Therefore, equation 15 is valid when equation 11 is true. When the clutch 
is fully engaged, one of the state variables, $\omega_{e}$, is lost. By eliminating $\omega_{e}$, and using equation 12, the following state equation is developed:

$$
\left[\begin{array}{c}
\dot{p} \\
\dot{v} \\
\dot{m}_{f}
\end{array}\right]=\left[\begin{array}{c}
v \\
\left(\tau_{e}\left(\frac{v \eta}{r}\right) \eta \gamma u / r-\left(C_{D} v^{2}+C_{V} v+F_{r r}\right)\right) / m_{v} \\
\omega_{e} \tau_{e}\left(\frac{v \eta}{r}\right) B S F C\left(\frac{v \eta}{r}\right) u
\end{array}\right]
$$

Notice that the system is nonlinear and time-invariant. The system could be linearized by calculating its Jacobian matrix[1], however the system does not stay within a small range of its state variables. In particular, the engine speed varies between 0 to about $7000 \mathrm{rpm}$, and the vehicle speed can vary between 0 to $40 \mathrm{ft} / \mathrm{s}$. There are a few nonlinear functions that depend on these 2 variables, so linearizing them would reduce accuracy significantly. Therefore, classic optimal control theory cannot be applied[1], and the nonlinear system must be simulated using Simulink.

\subsection{Simulation Development}

Figure 2 shows the highest level of the simulation. The main plant model has 1 input and 4 outputs. The driver input is controlled by a Simulink block with hysteresis. The input to the block is the vehicle speed, and the output is either 0 or 1 . When the vehicle speed is below the minimum speed (a constant), the output switches to 1 until the maximum speed (another constant) is reached. The output then switches to 0 until the minimum speed is reached again. 

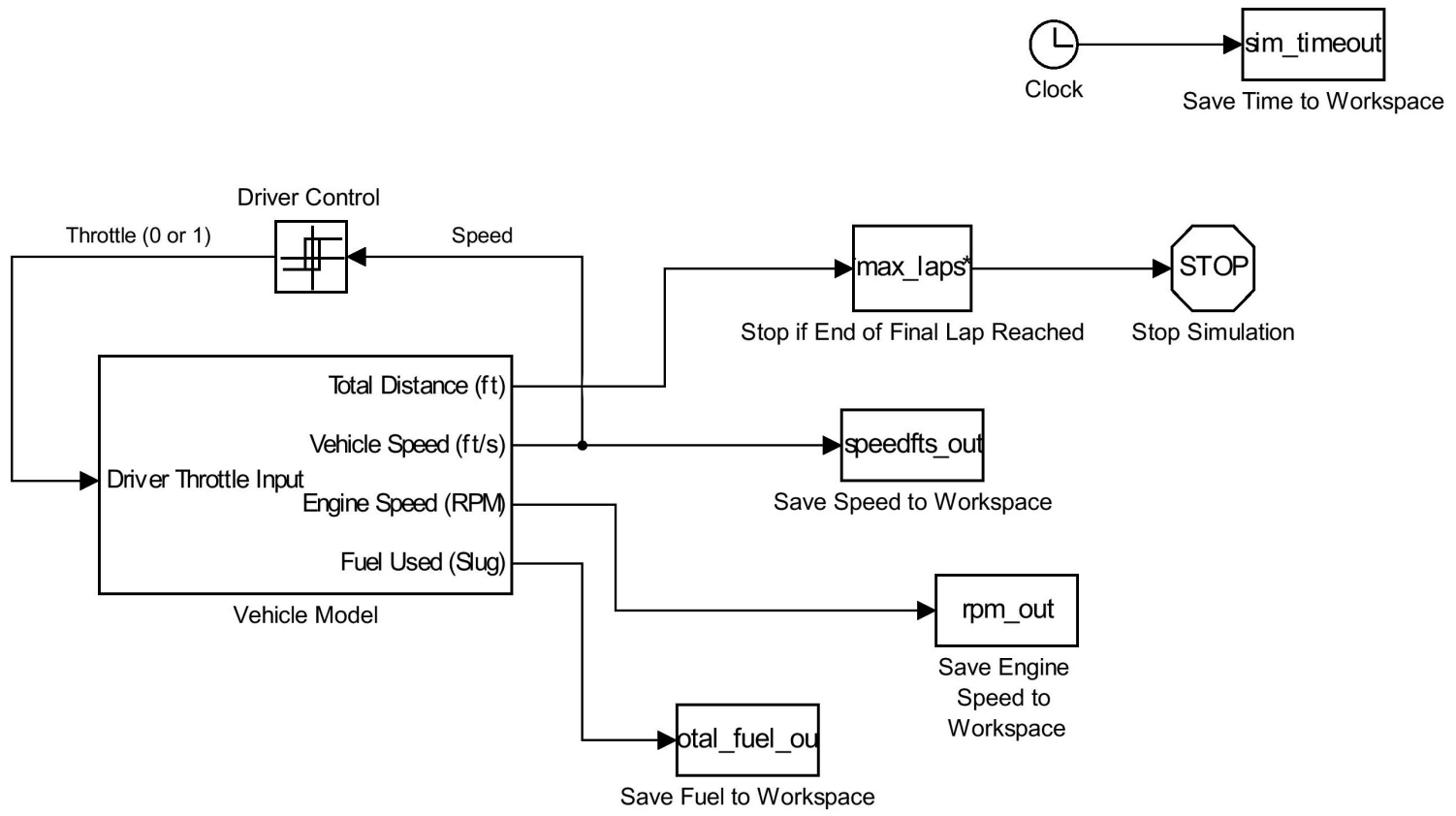

Figure 2: Top-Level View of Simulink Model

The simulation stops after the total distance for the competition has been travelled. For the current competition, this distance is 6 miles. The remaining blocks on this level simply save the outputs of the simulation and put them into another MATLAB workspace.

Figure 3 shows the inside of the plant shown in Figure 2. This level handles the integration of vehicle acceleration to get speed and position. Additionally, speed goes into the Lap Calculation subsystem to calculate the current position of the vehicle on the track. This subsystem is shown in Figure 4 


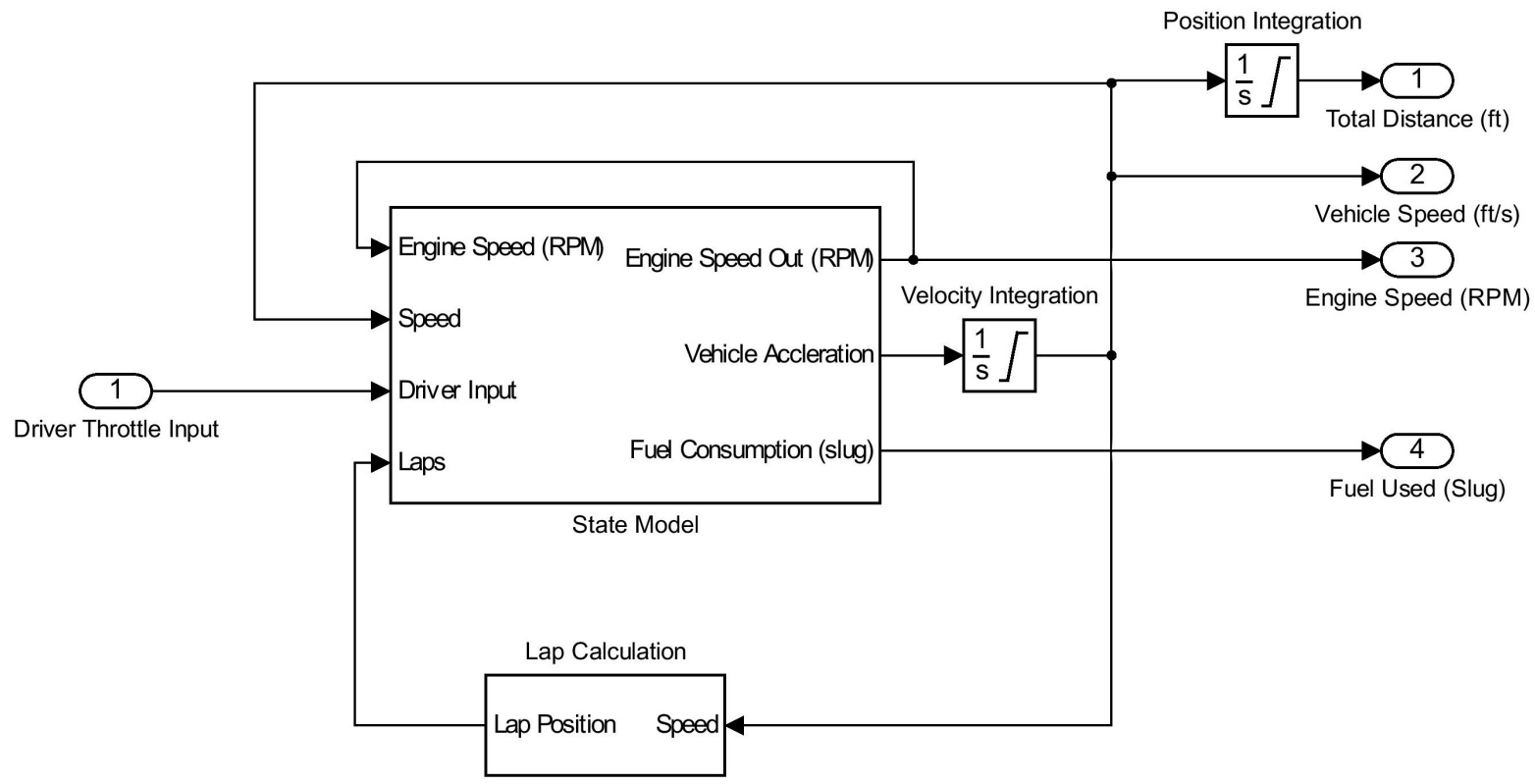

Figure 3: Plant Subsystem of Simulink Model

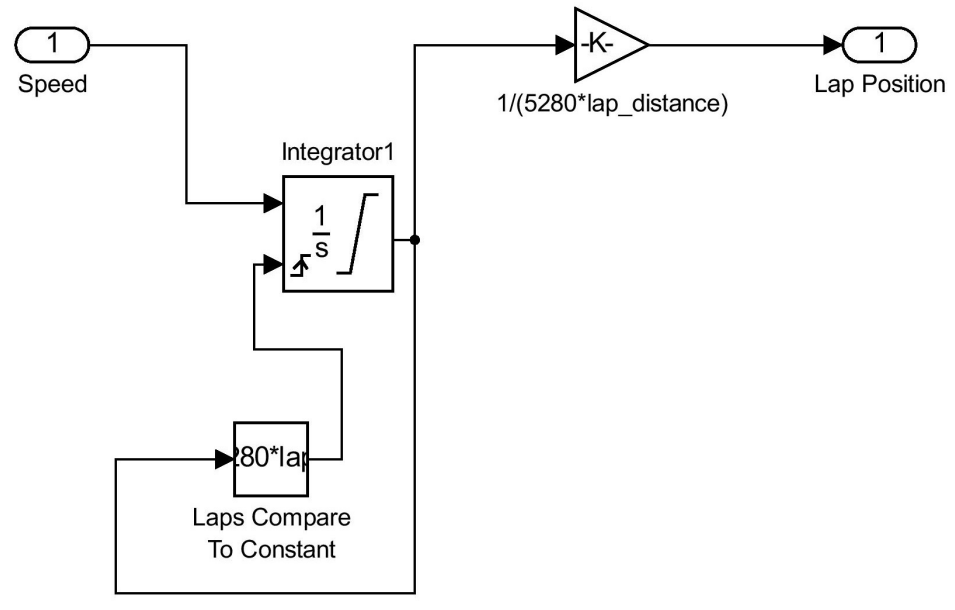

Figure 4: Lap Calculation Subsystem

Speed enters the Lap Calculation subsystem and is integrated to obtain distance travelled in feet. This output is compared to the length of a lap in feet. If the distance is greater than the total distance of a lap, the integrator is reset to 0 . The 
distance in feet is converted to lap position by dividing by the length of a lap in feet. The lap position output stays between 0 and 1 , where 0 is at the start of a lap, and 1 is at the end of a lap.

Figure 5 shows the inside of the State Model subsystem shown in Figure 3. The upper half of this level is dedicated to calculating torque produced by the engine/clutch and determining how much fuel is used. When the driver input is 0 , there is no torque from the drivetrain on the vehicle, and no fuel is consumed. The nonengine forces acting on the vehicle come from the road load, which is calculated in the Road Load Forces subsystem, shown in Figure 6. Drivetrain torque is converted to rear-wheel force through Equation 3. Then, the force from the engine is subtracted from the road load force and then divided by the total mass of the vehicle to get vehicle acceleration. 


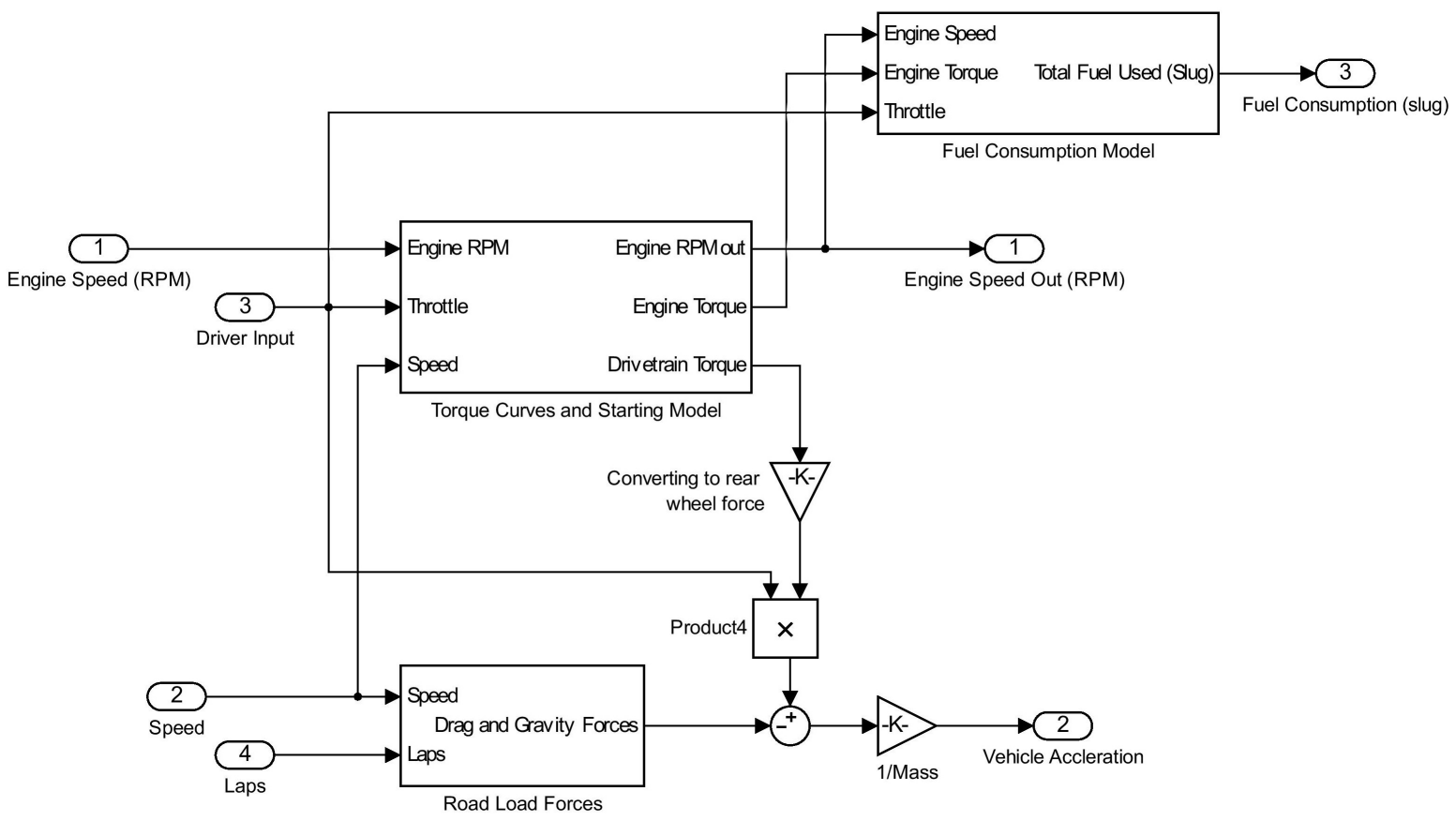

Figure 5: State Model Subsystem

Figure 6 shows how the road load forces are calculated in the Road Load Forces subsystem. Speed enters a lookup table generated from the first 3 terms of the road load equation(2), which outputs the road load force. The lap position (0 to 1) enters a lookup table that represents the last term of the road load equation(2), which outputs the road load force from the slope of the track. 


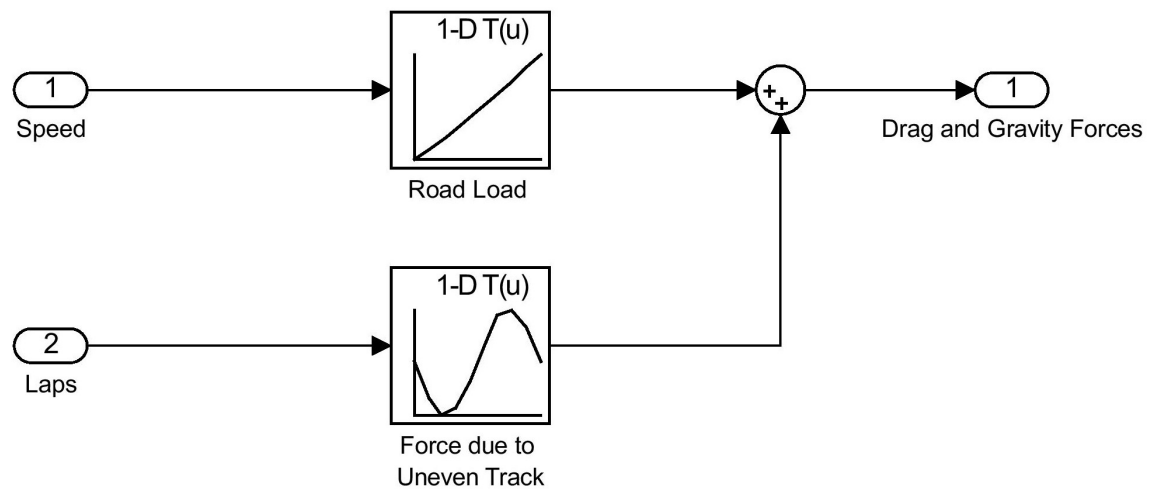

Figure 6: Road Load Forces Subsystem

Figure 7 shows the Fuel Consumption Model subsystem. If the input engine speed is less than $\omega_{i}$, the fuel consumption rate is constant. When engine speed is above $\omega_{i}$, engine speed is used as an input into a BSFC table. The output is in units of $l b m /(h p * h r)$. This is multiplied by engine power in units of $l b f * f t * r p m$. This is converted to slug/s by dividing the output by $g * 3600 * 5252$. That output is multiplied by the input (so the output is nonzero only when the input is 1) and integrated to get total fuel consumed in slugs. 


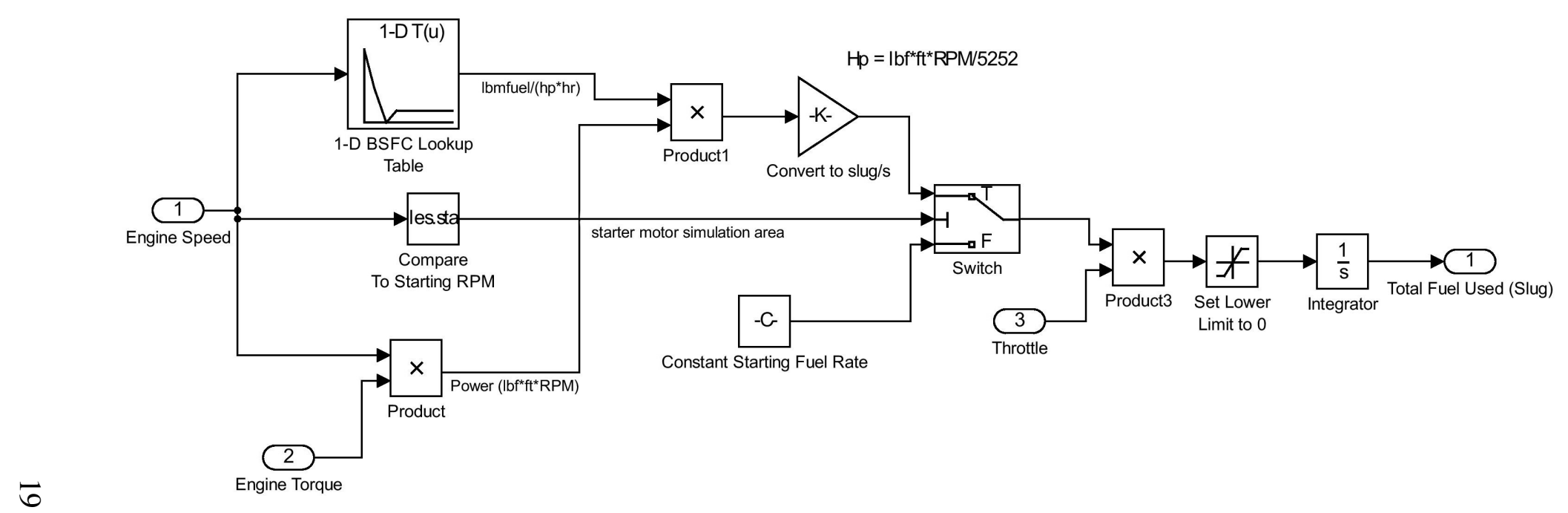

Figure 7: Fuel Consumption Model Subsystem 
Figure 8 shows the inside of the Torque Curves and Starting Model subsystem. In the bottom left of the figure, engine speed is compared to $\omega_{i}$. If the engine speed is less than idle, the engine acceleration is a constant. Otherwise, engine speed is used as an input to the engine torque lookup table. The clutch torque is calculated from the Clutch Curve subsystem table, which is subtracted from engine torque, as in Equation 9. That torque is then divided by $J_{e}$ to get engine acceleration in $\mathrm{rad} / \mathrm{s}^{2}$. The engine acceleration is integrated through an integrator block that resets to 0 when the driver input changes from 0 to 1 . The engine speed output from the integrator is converted to rpm, and then it is output if the clutch is not fully engaged. If the clutch is fully engaged, the engine speed is calculated from vehicle speed using Equation 12. In the upper right corner of the figure, the output torque to the drivetrain is switched depending on whether the clutch is fully engaged or not. 


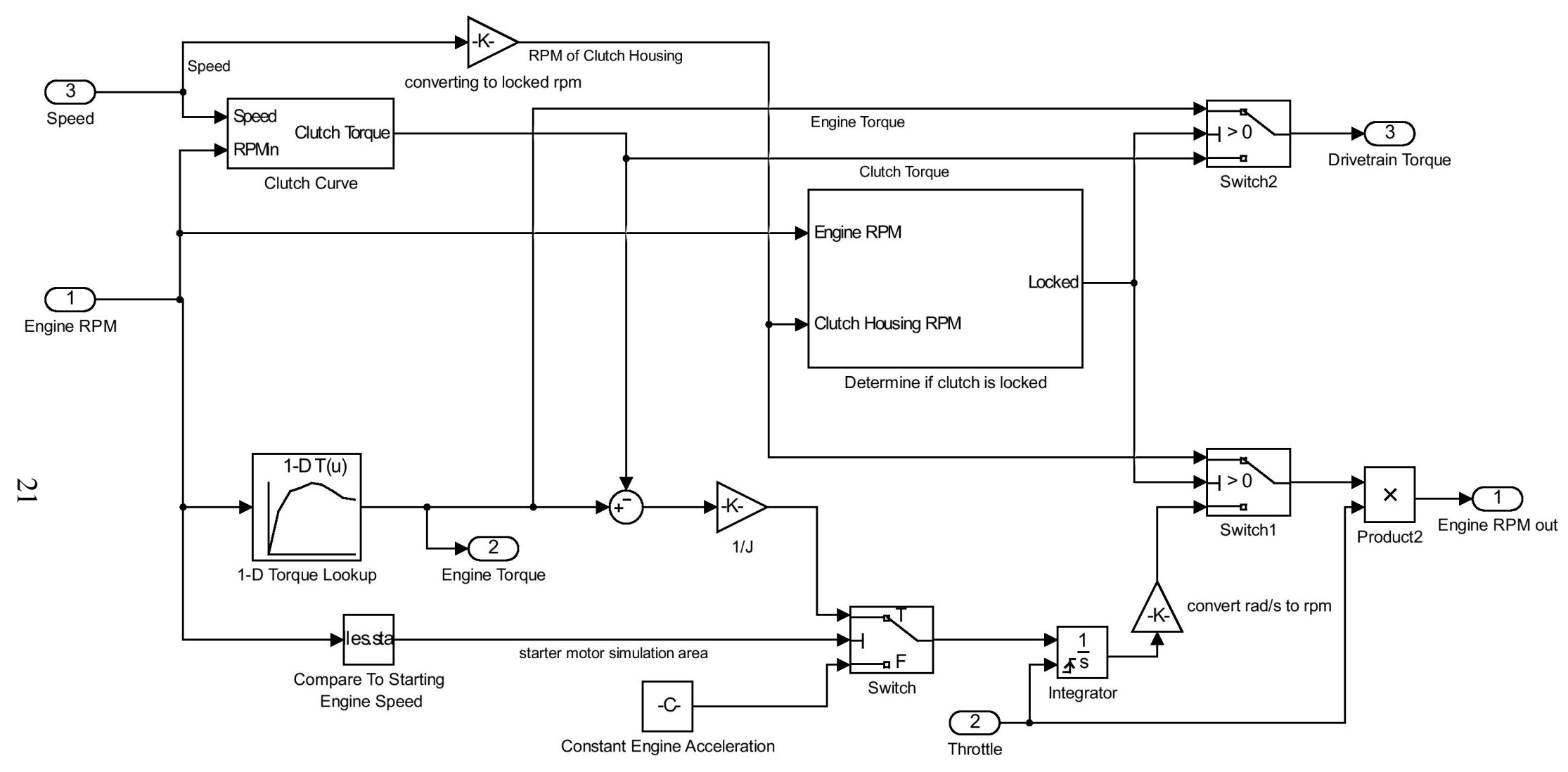

Figure 8: Torque Curves and Starting Model Subsystem 
Figure 9 shows the inside of the Clutch Curve subsystem. At the bottom of the subsystem, engine speed is used to calculate clutch torque using a lookup table generated from Equation 10. The upper part of the subsystem calculates the difference between the engine speed and clutch housing speed. If the engine speed is less than the clutch housing speed, no torque should be placed on the drivetrain, so the friction term is 0 . Once engine speed is greater than the clutch housing speed, the friction term is 1 .

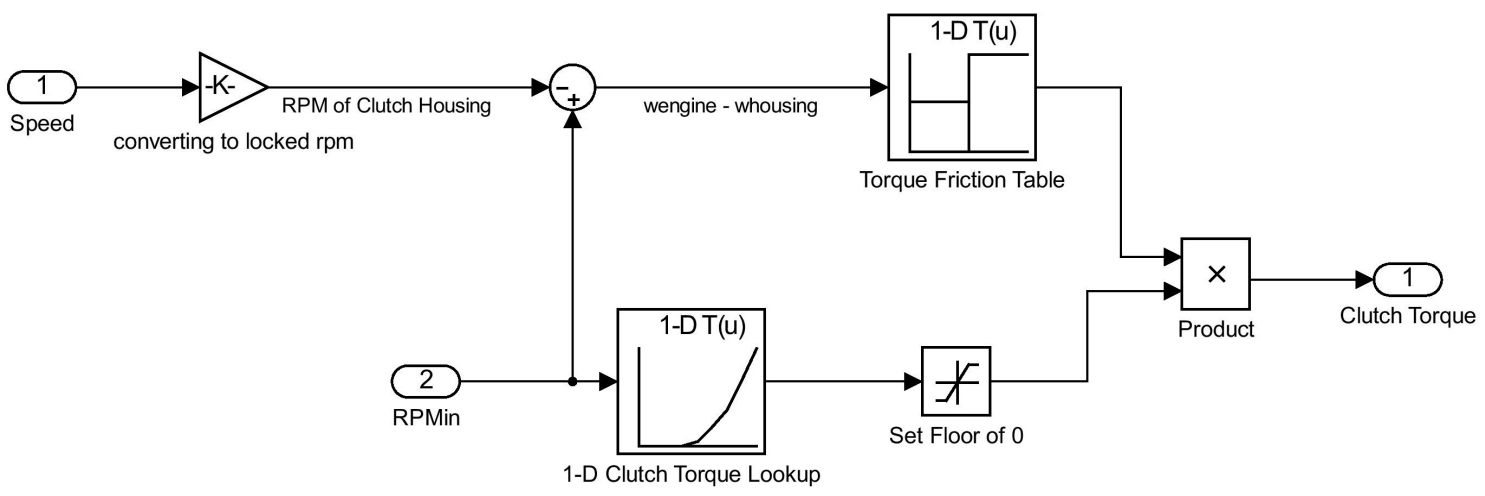

Figure 9: Clutch Curve Subsystem

Figure 10 shows the inside of the Clutch Locking subsystem. At the bottom of the subsystem, the engine speed is compared to the clutch locking speed. This clutch locking speed is calculated before a simulation begins by determining the engine speed at which clutch torque equals engine torque. If the engine speed is much less than the locking speed, the clutch will not be engaged. If the engine speed is close to the locking speed, the top part of the subsystem is used. The magnitude of the difference between engine speed and clutch housing speed is determined and then multiplied by negative 1. It must be multiplied by negative 1 since Simulink's hysteresis block needs the top limit to be higher than the bottom limit. Once the 
difference between the clutch housing speed and engine speed becomes very small, the clutch is now "locked" and it will not unlock until the engine speed drops below the locking speed.

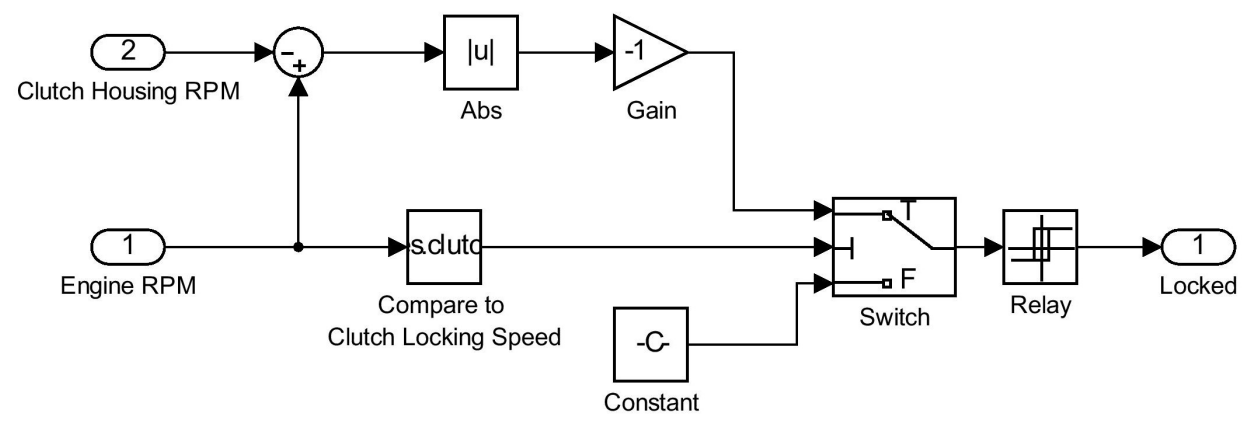

Figure 10: Clutch Locked Determination Subsystem

Once the simulation development is complete, data must be gathered to determine the various tables and coefficients. 
4 Analyzing Data to Build and Validate Model

There are 2 main methods of of determining the various coefficients and constants in the Simulink model.

One method is to use detailed equations and models that can predict those coefficients from several inputs. Examples include: the aerodynamic force coefficient could be determined by using computational fluid dynamics, engine torque and BSFC curves could be determined using detailed engine models, and clutch coefficients could be determined by analyzing the geometry of the clutch. The issue with this method is the inaccuracies associated with using more models and more inputs. Each model requires different assumptions that are not completely true, and each input carries a certain amount of uncertainty, which can be amplified after a complex calculation. Additionally, if the coefficients were determined this way, and the resulting simulation did not match up with real conditions, it would be difficult to determine which model or assumption was incorrect.

A more intuitive and accurate method is to gather data using on-vehicle data acquisition systems, and utilize that data to determine the various coefficients. This method can also account for some unpredictable dynamics. Also, it is difficult to dispute the accuracy of the coefficients when they were gathered from actual data, not from simplified models and equations. For these reasons, the second method was chosen moving forward. 


\subsection{Data Acquisition System}

The primary approach to gather data is using the RaceCapture[7] data acquisition system. Figure 11 shows RaceCapture mounted on the firewall of the Supermileage vehicle.

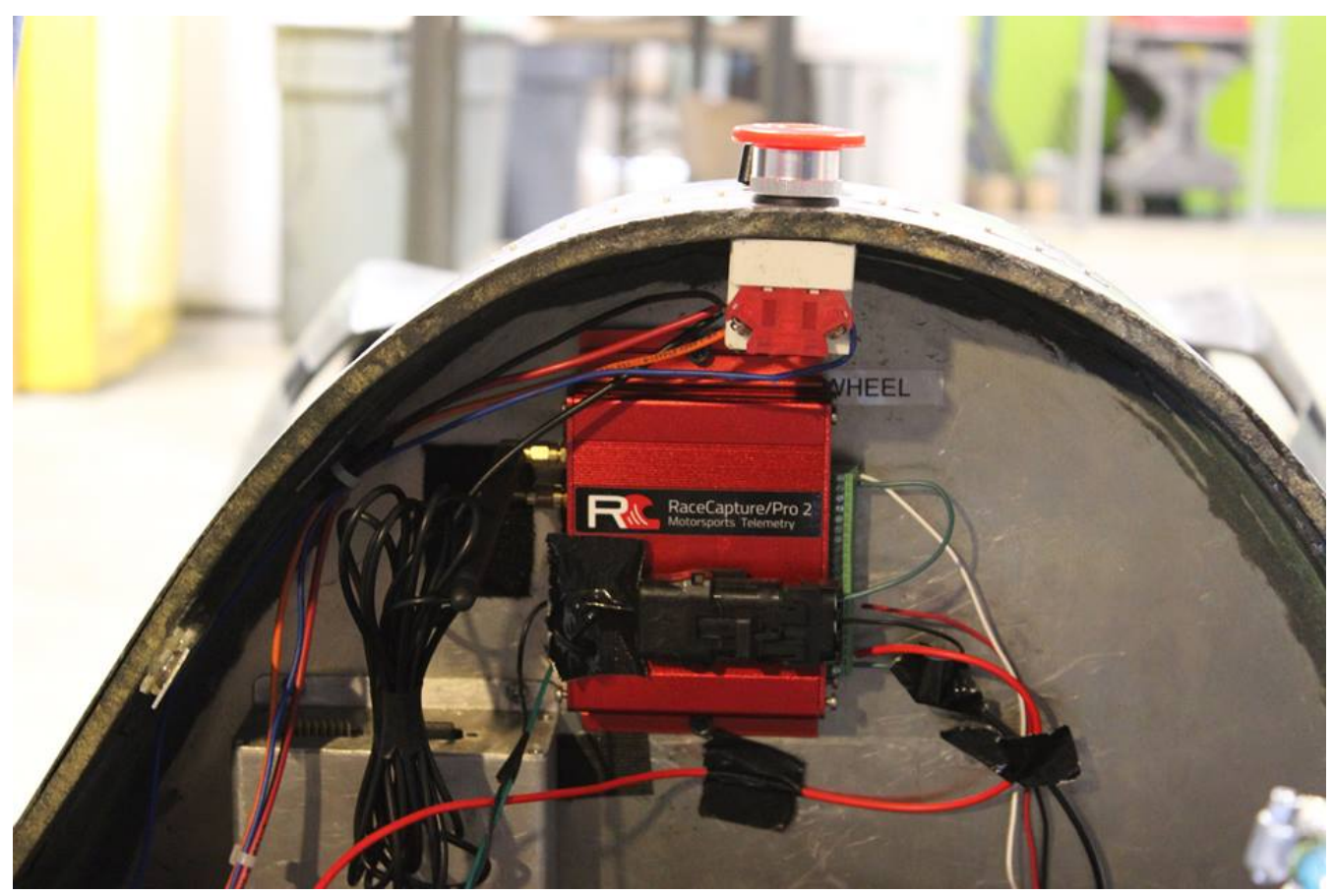

Figure 11: RaceCapture in Vehicle

RaceCapture has a few built-in sensors, and also has inputs for external sensors. Additionally, it has a CAN port, which is used to capture data from the Supermileage engine control unit. This data is logged to a micro-SD card and processed later using MATLAB. This data was gathered at a sampling rate of $50 \mathrm{~Hz}$. A list of some of the important data logged is shown below:

- Acceleration (from 3 axis accelerometer) 
- Roll, Pitch, Yaw (from 3 axis gyroscope)

- Engine Timing Advance

- Air to Fuel Ratio

- GPS (Latitude, Longitude, Altitude)

- Battery Voltage

- Engine Coolant Temperature

- Fuel Injector Pulse Width Duration

- Engine Speed

- Vehicle Speed (from GPS data)

- Time

Vehicle speed is one of the most important parameters that is measured, however it had to be measured by differentiating position data from the GPS sensor. RaceCapture has a built-in algorithm to determine speed, which has filtering and generally works fairly well. There is more noise in the output than there would be if a rear-wheel Hall effect sensor was used, but it was not feasible or necessary at the time to install one.

Figure 12 shows an example of speed logged using RaceCapture. Raw speed data is logged in blue, while filtered speed is shown in red. The data is filtered using an FIR moving average filter of length 100. An FIR filter was used so the time delay 
associated with the filter is constant, and the large number of terms was used since any useful dynamic frequencies are at very low frequencies.

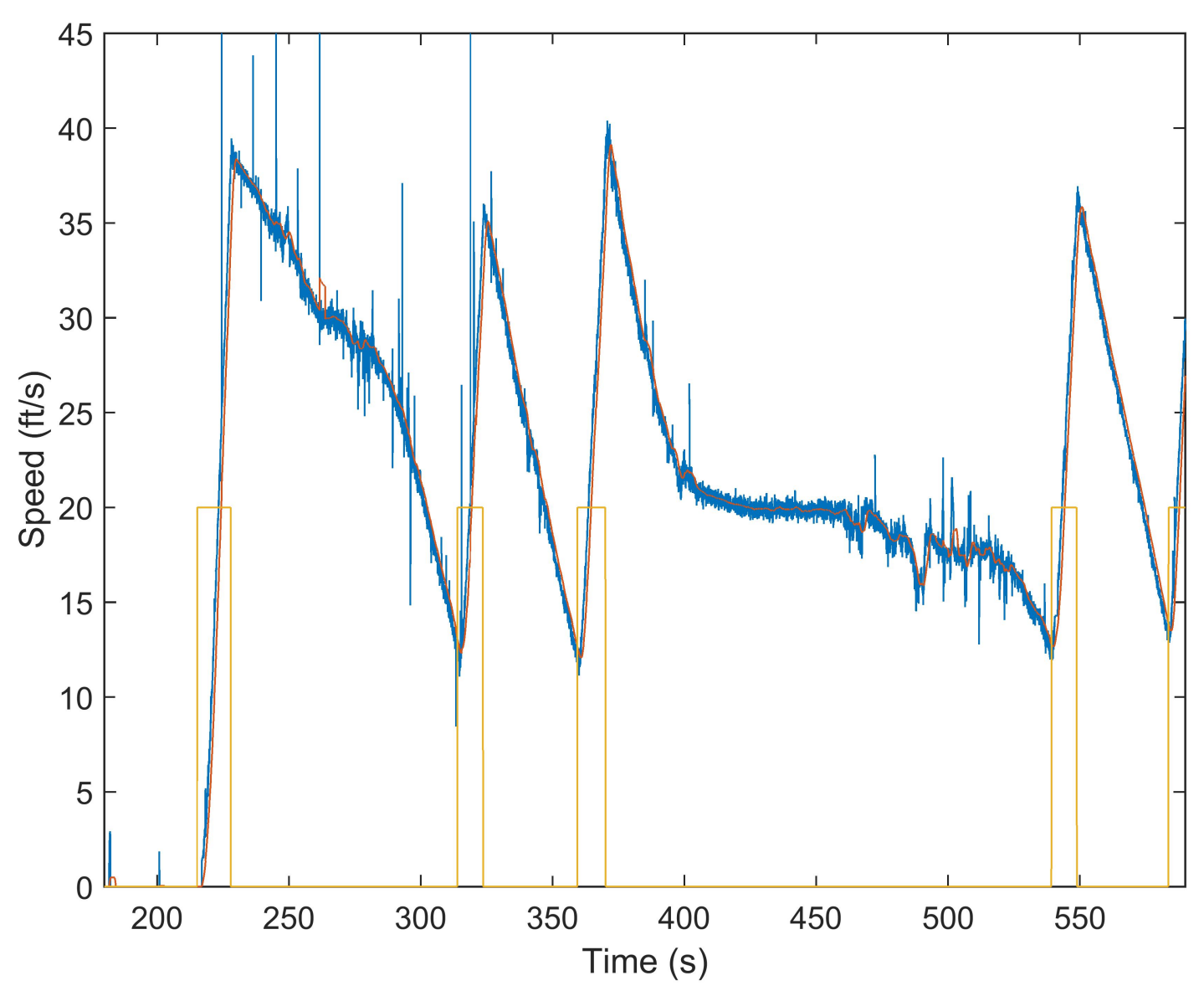

Figure 12: Speed Data Gathered from RaceCapture

In order to properly analyze the logged data, a simple algorithm was developed to automatically identify when the driver input was 0 or 1 . Essentially, if the engine speed is above 0 , and the engine speed is increasing, the driver input is 1 at that moment. Otherwise, the driver input is 0 . The driver input is plotted in yellow in Figure 12. Note that the input of 0 or 1 has been multiplied by 20 to scale better 
with the rest of the data in Figure 12.

Figure 13 shows an example of engine speed and pulse width data taken from the same run as Figure 12. This type of data will be used in the following sections to calculate various coefficients and verify the simulation.
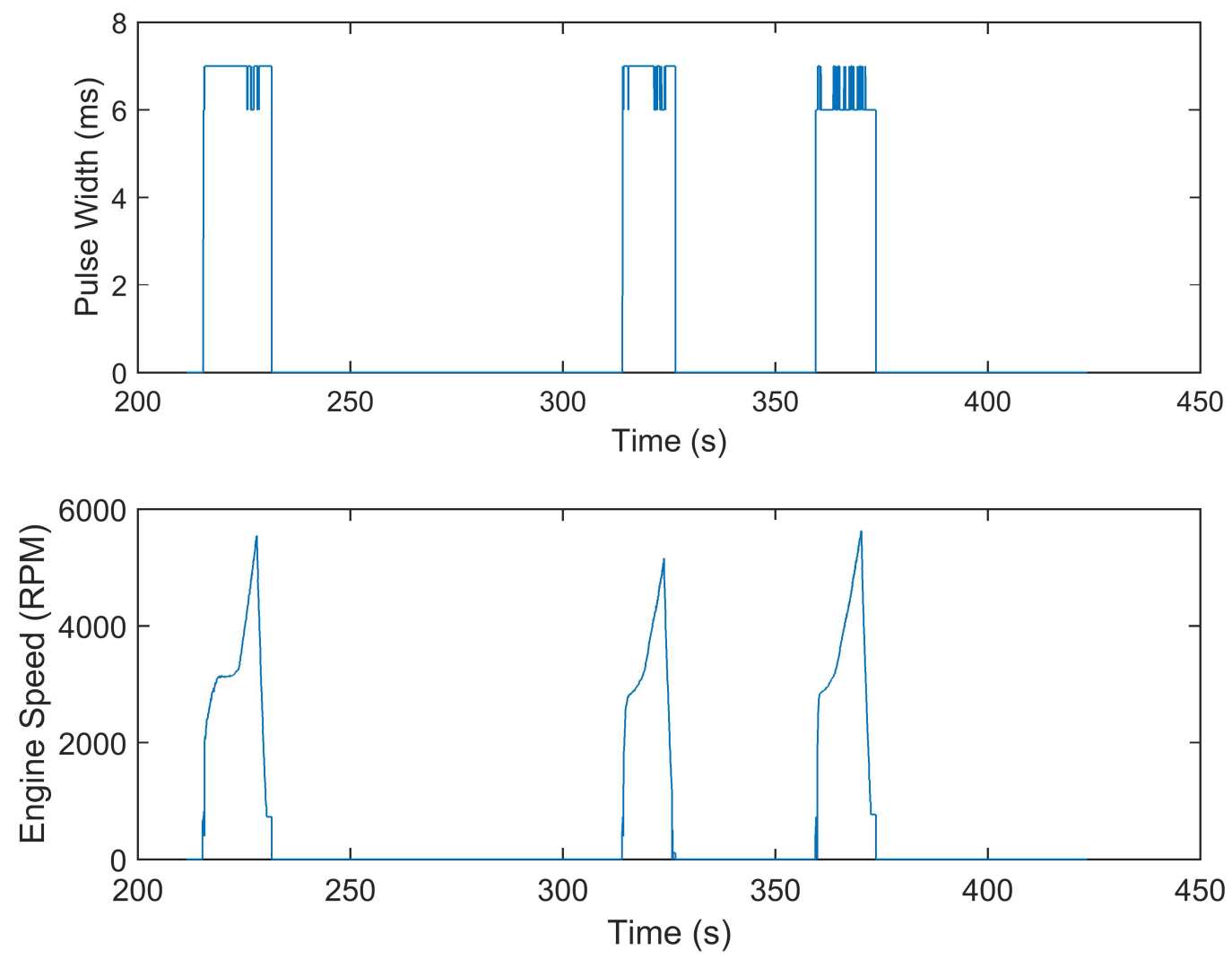

Figure 13: Engine Speed and Pulse Width Data Gathered from RaceCapture

\subsection{Road Load Coefficients}

The first important parameters to determine are the road load coefficients. Various studies have used coastdown testing to determine road load coefficients with a fair amount of accuracy[8]. When the vehicle is coasting, only the road load forces 
are acting on it. Therefore, to determine the coefficients, only the coasting down portions of the data will be considered. The vehicle must also travel at very high and very low speeds to see significant differences in road load force. The most straightforward way to measure road load force would be to use the RaceCapture accelerometer and multiply the measured acceleration by the mass of the vehicle to get force. However, RaceCapture is mounted very close to the engine, so vibrations make the accelerometer data much too noisy to measure small changes accurately. Therefore, speed must be used directly to measure road load.

Acceleration can be attained by using polynomial regression to fit a polynomial to speed as a function of time. This polynomial can then be differentiated to get acceleration as a function of time. Road load force is a second-order polynomial function of speed if the slope of the track is $0^{\circ}$ (Equation 2). For this reason, acceleration can be fit to a second-order polynomial of speed, and then multiplied by total vehicle mass to get road load force as a function of speed. Equations for this process are shown below:

$$
\frac{d}{d t}\left(\operatorname{poly}\left(\underline{t}_{R C}, \underline{v}_{R C}\right)\right) \rightarrow \dot{v}(t)
$$

Where:

$\underline{t}_{R C}$ is the time vector gathered from RaceCapture $(s)$

$\underline{v}_{R C}$ is the speed vector gathered from RaceCapture $(\mathrm{ft} / \mathrm{s})$

$\operatorname{poly}(x, y)$ is the polynomial regression function

$$
\rightarrow m_{v} \cdot \operatorname{poly}\left(\underline{v}_{R C}, \dot{v}\left(\underline{t}_{R C}\right)\right) \rightarrow F_{r l}^{\prime}(v)
$$


Where:

$F_{r l}^{\prime}(v)$ is the estimated road load force equation

The primary problem with this process is that a perfectly flat track is required, and Cal Poly Supermileage does not have access to a high-speed, flat track. As seen in Figure 12, coastdowns are not consistent due to variations in the track. A better way to visualize the uneven track is in Figure 14, which shows various coastdowns on different portions of the track. Notice the vehicle decelerates differently depending on where it is. Between 0 and 0.3, the vehicle is on a slight downhill. Between 0.4 and 0.9 , the vehicle is on a slight uphill and decelerates faster. 


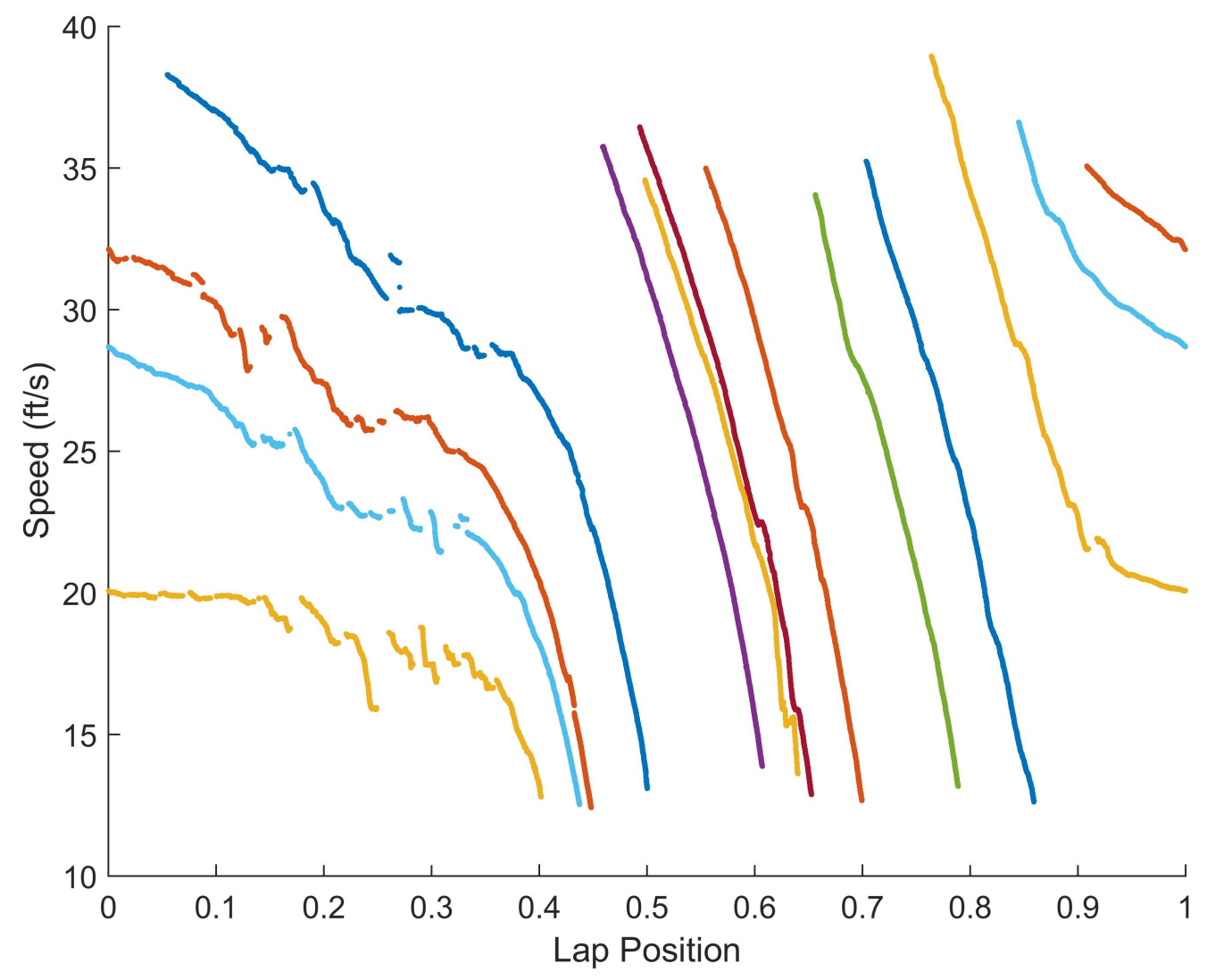

Figure 14: Logged Speed versus Lap Position

Since the track is uneven, the slope must be calculated at every point along the track to correct for it. Altitude data from GPS would be straightforward, however the altitude data gathered is noisy and inaccurate, shown in Figure 15. Even with heavy filtering, the data is too noisy to find small changes in slope. 


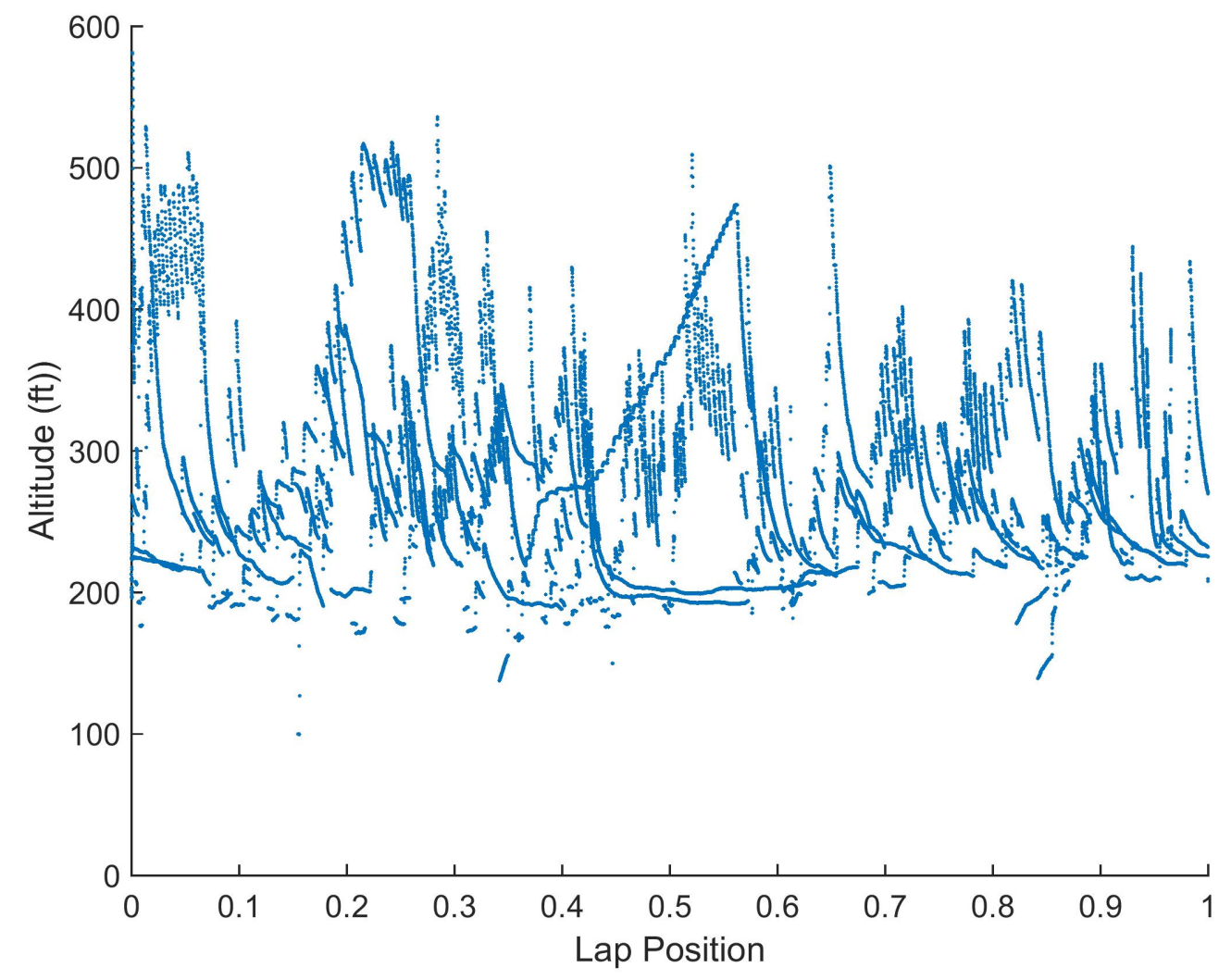

Figure 15: Altitude versus Lap Position

Therefore, an alternate source must be used to determine the slope of the track. Google Earth is an easy tool to use, and it provides an elevation profile along a track. However, the altitude accuracy from Google Earth is also limited. It uses SRTM data to determine altitude, and SRTM has a $90 \%$ linear error probable of $98.4 \mathrm{ft}$ according to U.S. Military Specification [MIL]-PRF-89020B [18]. Google Earth data for the high-speed Supermileage testing track in Lompoc, CA, is shown in Figure 16. 


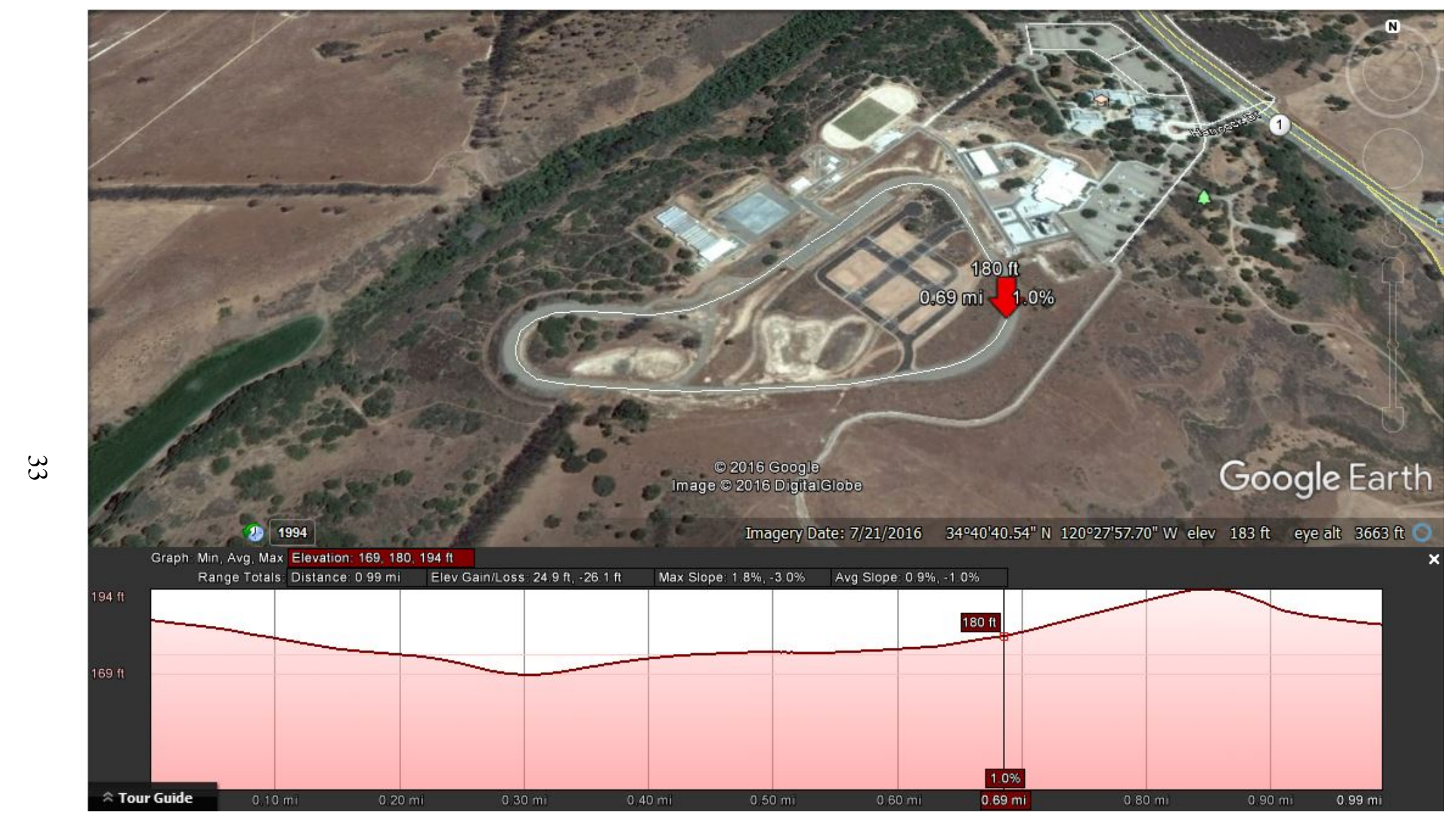

Figure 16: Elevation Profile of Test Track 
The maximum and minimum altitudes from this data are $169 \mathrm{ft}$ and $194 \mathrm{ft} \mathrm{re}-$ spectively, which seem more reasonable than the noisy GPS data. This data also matches the previous observation of a downhill portion from 0 to 0.3 laps, and an uphill from 0.4 to 0.9 laps. However, the intricate curves on the Google Earth data might not actually be there due to the inaccuracies. Additionally, the process shown in Equations 17 and 18 does not work if the slope is changing over time or distance. Therefore, a constant uphill and constant downhill slope will be assumed for the 2 main portions of the track. This way, Equations 17 and 18 can be followed, and then corrected using Equation 19.

$$
\hat{F}_{r l}(v)=F_{r l}^{\prime}(v)-m_{v} g \cdot \sin \left(\theta_{\text {const }}\right)
$$

Where:

$\hat{F}_{r l}(v)$ is the corrected, estimated road load equation

In order to have a constant slope, the uphill and downhill portions must be analyzed separately. However, when the vehicle is on a downhill section, it takes significantly longer to coast down to a low speed. As shown in Figure 14, most downhill coastdowns end up going into part of the uphill section of the track. For this reason, it is more accurate to simply use the coastdowns from 0.5 to 0.9 lap position, where the vehicle is constantly going uphill. From 0.5 to 0.9 lap position, the vehicle increases $18 \mathrm{ft}$ in altitude (from Google Earth), and it travels $1848 \mathrm{ft}$. This corresponds to a slope of about $1 \%$ or $0.56^{\circ}$, which is equal to about $2.4 l b$ of force on the vehicle. Now, Equations 17 and 18 can be applied. 


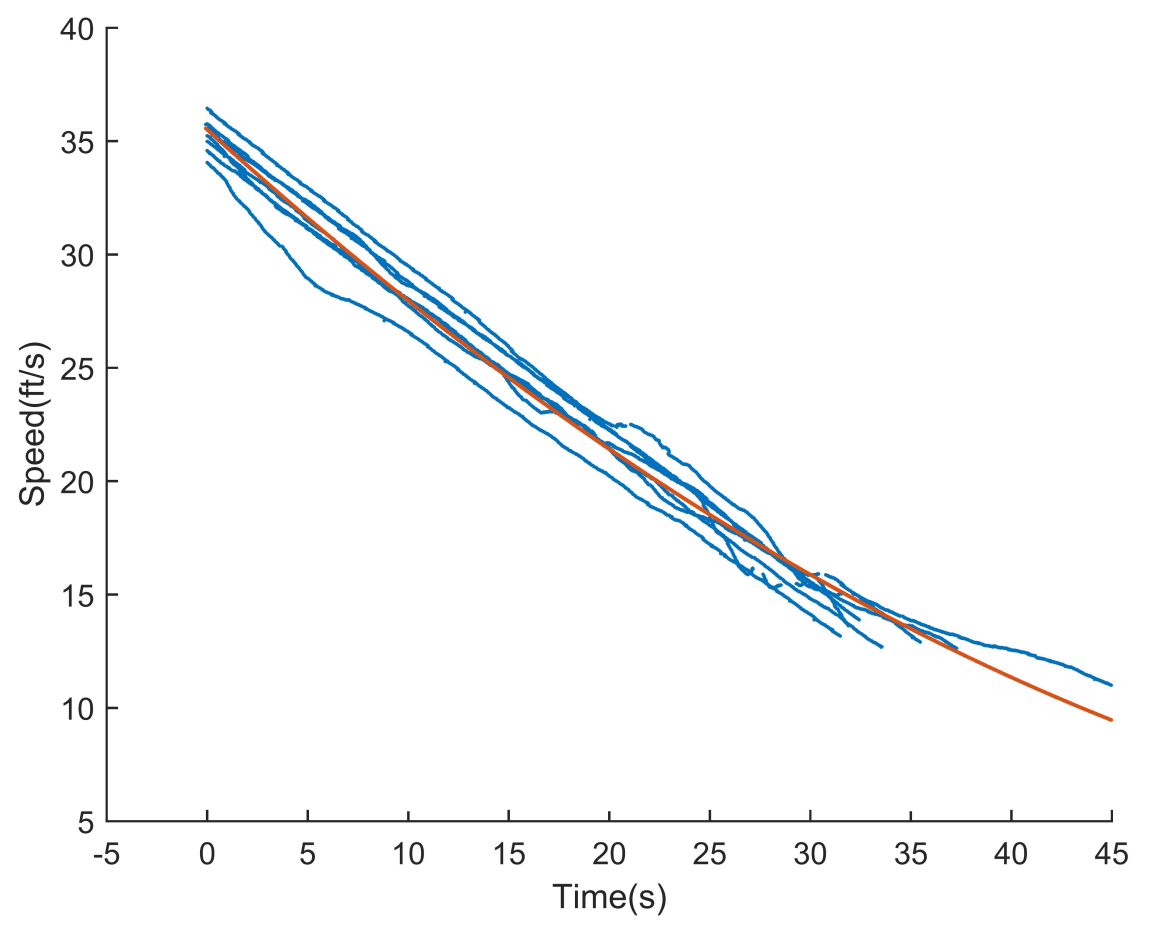

Figure 17: Coastdown Velocity Data from 0.5 to 0.9 Lap Position

Figure 17 shows all coastdown data on the uphill portions put on the same timescale. The blue lines are the various coastdowns, while the red line is the best fit curve, poly $\left(\underline{t}_{R C}, \underline{v}_{R C}\right)$. After using the best fit line in Equations 18 and 19, the output is shown in Figure 18. The red line shows the second-order best fit polynomial, and the blue line is the raw acceleration versus speed data (which is beneath the red line). 


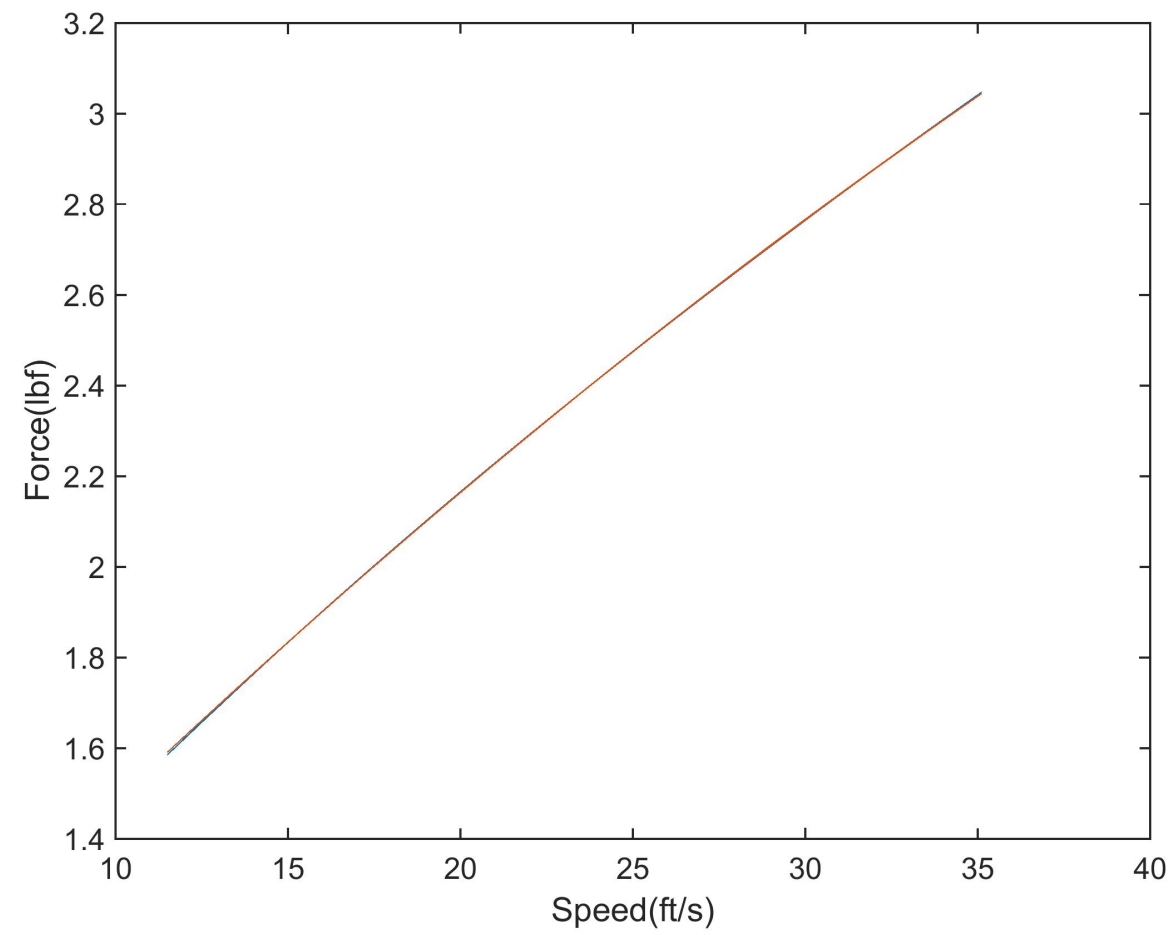

Figure 18: Road Load versus Vehicle Speed

The resulting coefficients are:

$$
\begin{aligned}
& C_{D}=-0.000393 \mathrm{lbf} /\left(f t^{2} / \mathrm{s}^{2}\right) \\
& C_{V}=0.0799 \mathrm{lbf} /(\mathrm{ft} / \mathrm{s}) \\
& F_{r r}=0.7243 \mathrm{lbf}
\end{aligned}
$$

Compared to the slope of the track, which applies $2.4 \mathrm{lbf}$ of force to the vehicle, the calculated $F_{r r}$ value of $0.7243 \mathrm{lbf}$ is fairly small. This means that any inaccuracies in slope calculation can have a dramatic effect on the final calculated $F_{r r}$ value. In the future, a more accurate way to measure road slope is necessary.

Also, notice that $C_{D}$ is negative, meaning the vehicle has negative aerodynamic drag. This is impossible and most likely caused by a combination of errors includ- 
ing changing wind speed and inaccurate speed measurements. The primary ways to get a more accurate drag number would be to install a rear-wheel Hall effect sensor and air speed sensor to more accurately capture vehicle and air speed. Additionally, Cal Poly Supermileage may have access to a wind tunnel to measure drag from a scaled-down model vehicle in the near future.

However, since a more accurate drag number is needed now, a different source will be used. Pac-car II, one of the most efficient vehicles ever made for the ShellEco Marathon, has an effective $C_{D}$ of $0.0002437 \mathrm{lbf} /\left(f t^{2} / s^{2}\right)$ [16]. This sets the standard as the lowest drag number in the Shell-Eco Marathon, and the Supermileage vehicle is likely not very close. Computational fluid dynamics analysis is being performed on the current vehicle, and it indicates that the $C_{D}$ of the Supermileage vehicle is about 3 times that of Pac-car II. The number may also be larger, since CFD will not capture many aerodynamic effects that come up in actual testing. For this reason, the number of $0.0007311 \mathrm{lbf} /\left(f t^{2} / \mathrm{s}^{2}\right)$ will be assumed for further analysis.

\subsection{The Affect of Weight on Road Load}

In order to accurately determine how weight affects fuel efficiency, its affect on the road load coefficients must be found. Theoretically, increasing weight only increases the last term of the road load equation, $F_{r r}$ [4], as shown in Equation 20.

$$
F_{r r}=f_{r r} m_{v} g
$$

Where:

$f_{r r}$ is the coefficient of rolling resistance 
$f_{r r}$ does increase slightly with weight due to increased tire contact patch, but it is assumed to be very small compared to the effect of increasing weight itself. Therefore, $F_{r r}$ should increase linearly with vehicle mass, $m_{v}$.

Under these assumptions, a test was performed with two different vehicle weights. Since only the $F_{r r}$ constant is affected, a low-speed coastdown test is sufficient. For the test, the vehicle was placed at the bottom of a slight uphill portion of the track. It was brought up to about $20 \mathrm{ft} / \mathrm{s}$ and then allowed to coast down to $0 \mathrm{ft} / \mathrm{s}$. The same method as shown in Equation 17 and 18 was used to determine the coefficients, except acceleration was fit to a first-order polynomial. This is because aerodynamic drag is negligible at lower speeds. This process occurred 3 times at the base vehicle weight, and 3 more times with $60.4 \mathrm{lb}$ added. The uphill track did not have to be corrected for, since only the difference in rolling resistance force needed to be found.

Figure 19 shows the results of this testing. 


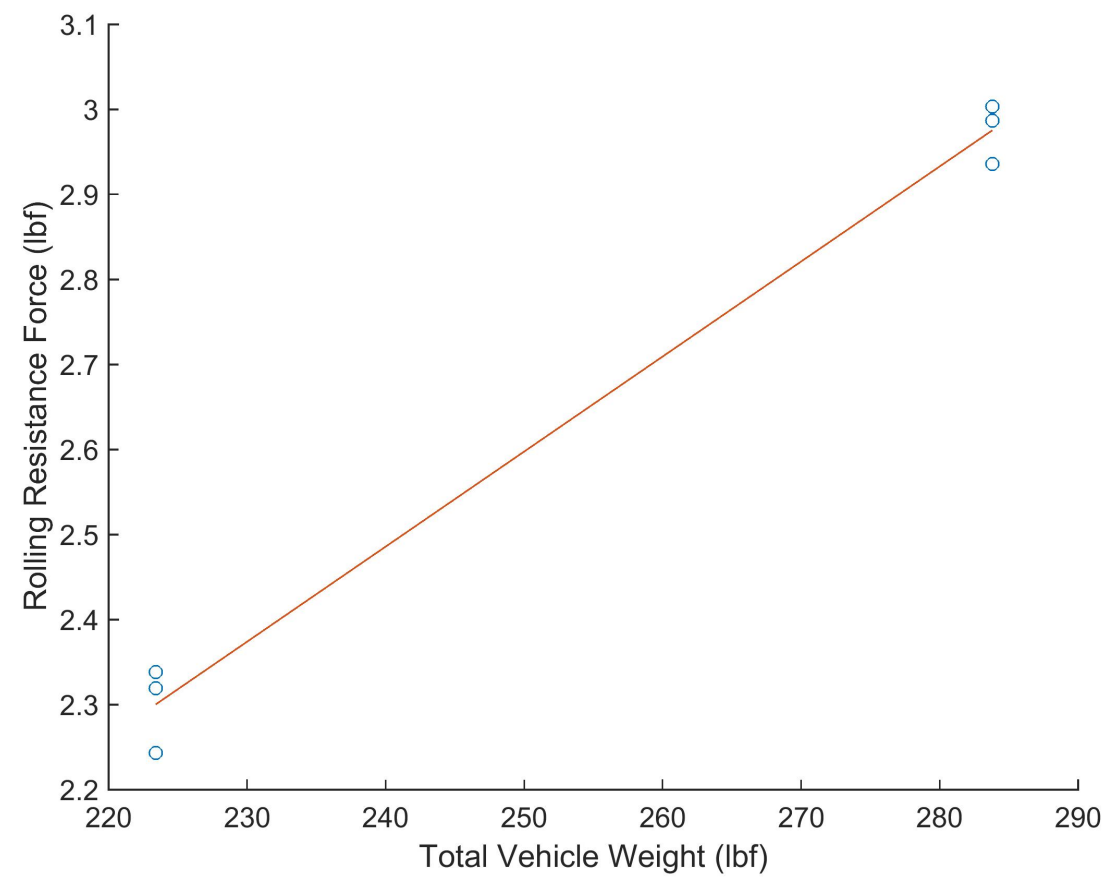

Figure 19: $F_{r r}$ versus Total Vehicle Weight

The standard deviation of the first 3 data points is 0.0502 , while the standard deviation of the last 3 are 0.0351 . The red line is the best fit curve for the data. The following equation is the best fit polynomial:

$$
\delta F_{r r}=0.3596 \cdot \delta m_{v}
$$

Where:

$\delta F_{r r}$ is the change from initial $F_{r r}$

$\delta m_{v}$ is the change from initial $m_{v}$

To calculate a new $F_{r r}$ from an old $m_{v}$ and $F_{r r}$, Equation 22 can be used, which is derived from Equation 21. 


$$
F_{\text {rr new }}=0.3596 \cdot\left(m_{v \text { new }}-m_{v \text { old }}\right)+F_{r r \text { old }}
$$

\subsection{Engine Torque and BSFC Curves}

It is possible to determine engine torque and BSFC curves from RaceCapture data, however these are more accurately determined on an engine dynamometer. A previous study determined the engine curves for the current engine and tune[5]. The BSFC data can be verified by looking at the fuel pulse duration and engine speed data.

First, the fuel consumption rate is calculated from data using Equation 23.

$$
\dot{m}_{f}(t)=\omega_{e}(t) /(2 \pi) / 2(\text { cycle } / s) \cdot f_{P W}(t)(\text { fuel } m s) \cdot \dot{m}_{i}(m L / \text { fuel ms })
$$

Where:

$f_{P W}$ is the injector pulse width in fuel $\mathrm{ms}$

$\dot{m}_{i}$ is the fuel injector flow rate $m L / f u e l ~ m s$

The theoretical fuel flow rate can be calculated using Equation 6. Figure 20 shows the theoretical fuel flow rate versus engine speed in black. The blue points are experimental data, and the red line is the best fit polynomial for the experimental data. 


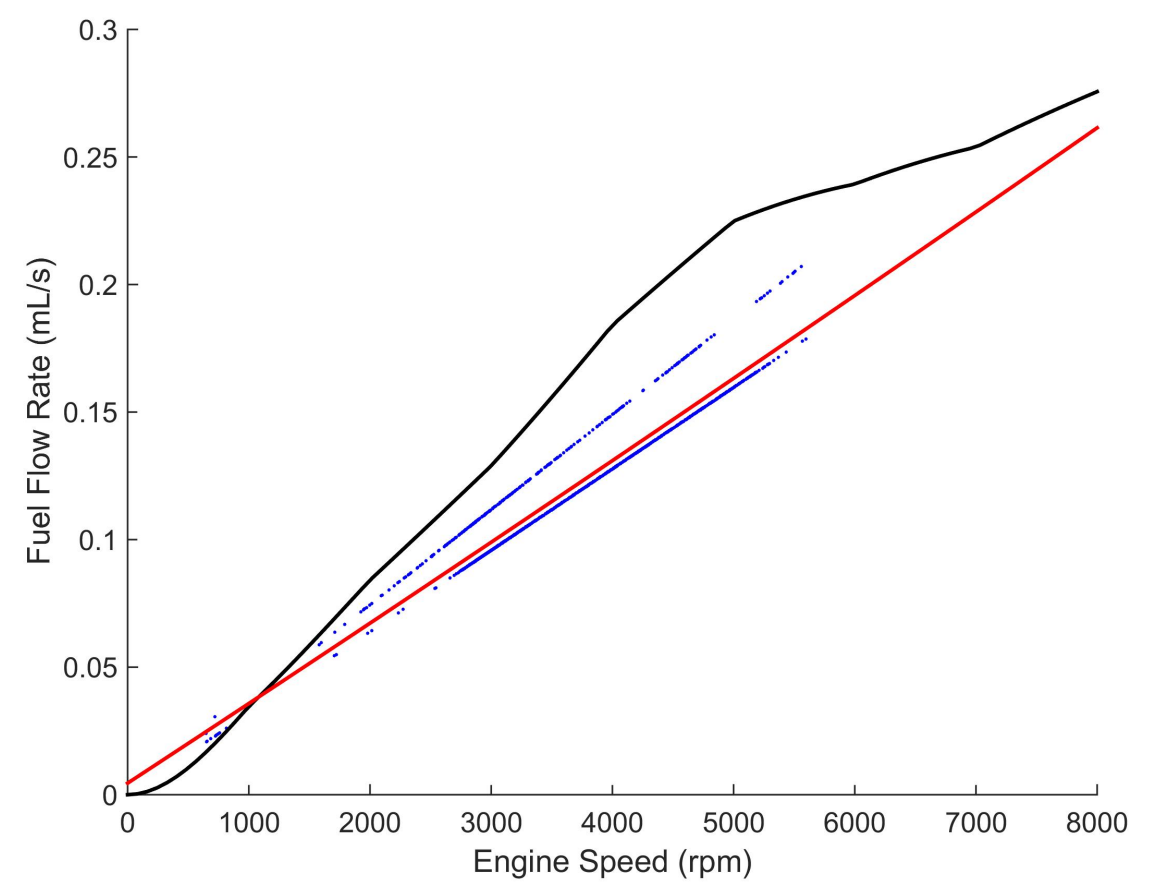

Figure 20: Fuel Flow Rate versus Engine Speed

Notice that the theoretical fuel consumption is somewhat higher than the actual fuel consumption. However, it still follows the trend of the experimental data. The injector pulse width was nearly constant during testing, so fuel flow rate has a linear relationship with engine speed. For the rest of the analysis, the best fit polynomial from the experimental data will be used for more accurate results.

The torque data cannot be verified using the available data; it is used to determine drivetrain efficiency in the next section.

\subsection{Drivetrain Efficiency}

Typically, to determine drivetrain efficiency, a chassis dynamometer is used. Other studies have used a chassis dynamometer to successfully measure drivetrain effi- 
ciency from a Shell Eco-marathon vehicle[3]. This is the best way to acquire accurate data. However, no chassis dynamometer was available at the time of this study, so data gathered from RaceCapture will be used. To determine the drivetrain efficiency, only the data while $u=1$ is used. Figure 21 shows how engine speed changes with time; each color is from a different burn.

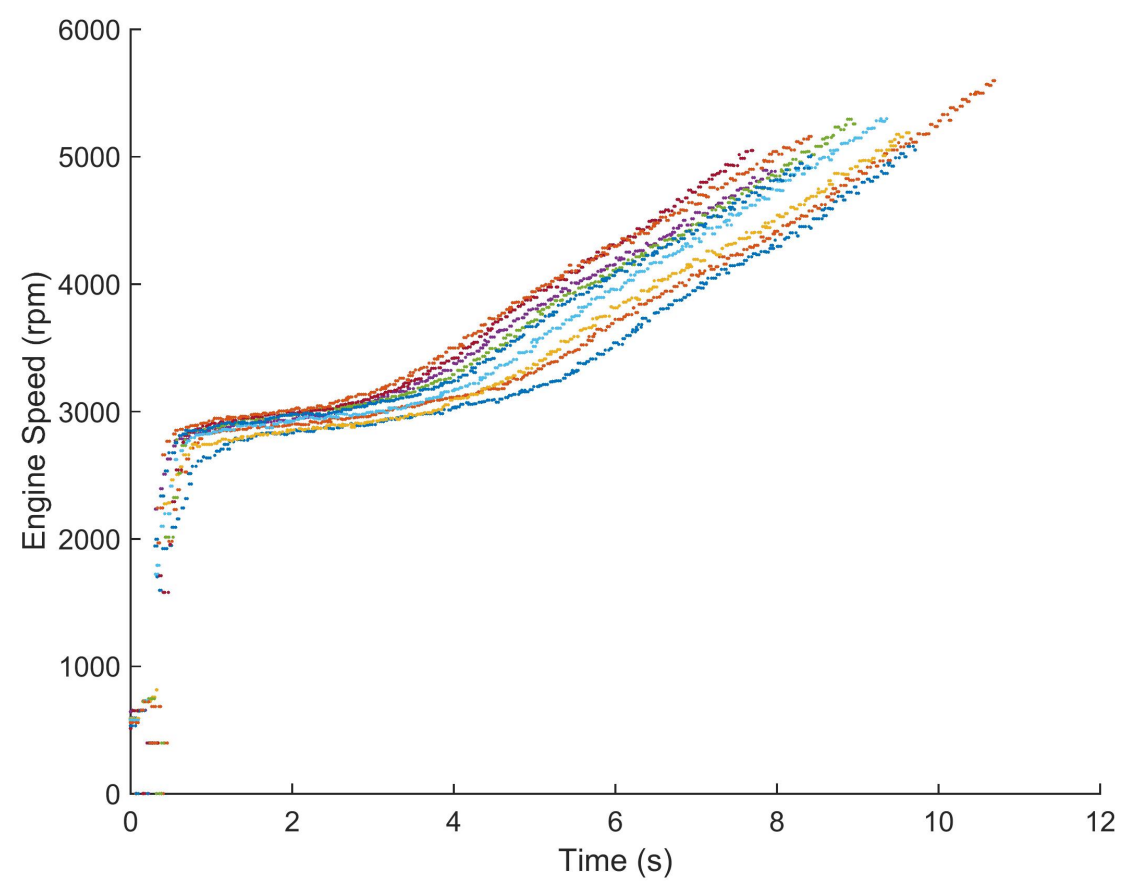

Figure 21: Engine Speed versus Time

Figure 22 shows the clutch housing speed over time. This speed was calculated using Equation 12 from the filtered GPS speed data. Notice that the engine speed and clutch housing speed nearly match around 4 seconds on. This is when the clutch is fully locked and engaged. Therefore, for the efficiency analysis, only the data after the 4 second mark will be used. For the clutch torque determination (next section), the data before the clutch is fully engaged will be used. 


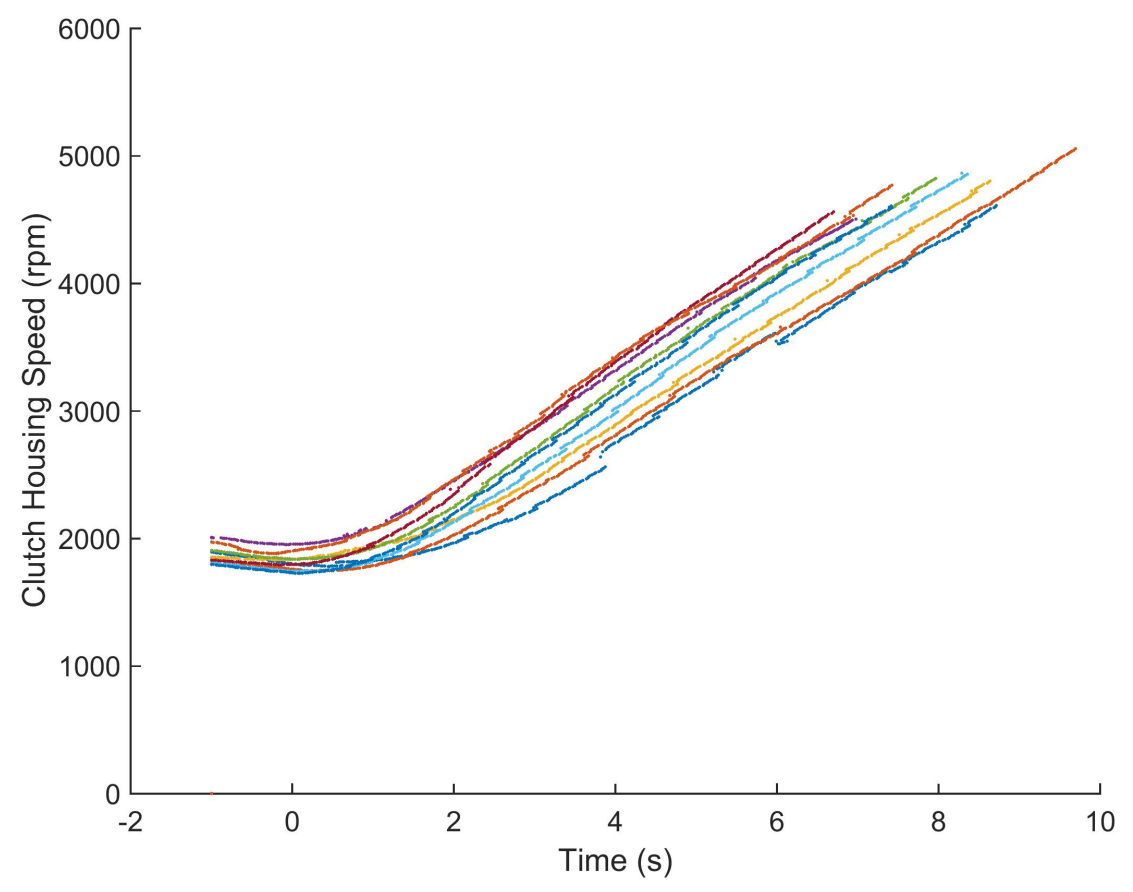

Figure 22: Clutch Housing Speed versus Time

Using the engine torque data from the previous study[5], it is possible to calculate the theoretical acceleration on the rear wheel (assuming $100 \%$ efficiency), by using the following equation:

$$
\dot{v}_{t h}=\left(\frac{\tau_{e}\left(\omega_{e}\right) \eta}{r}-F_{r l}\right) / m_{v}
$$

Where:

$\dot{v}_{t h}$ is the theoretical vehicle acceleration

Notice that the equation requires an accurate road load force, so the road load found previously will be used.

To get the actual acceleration, the discrete derivative can be used on the filtered 
GPS speed data. There is no need to use least squares regression as before, since the magnitude of the acceleration when burning is much larger than the sensor noise.

Figure 23 shows the theoretical vehicle acceleration in black, while the rest of the colors are experimental acceleration.

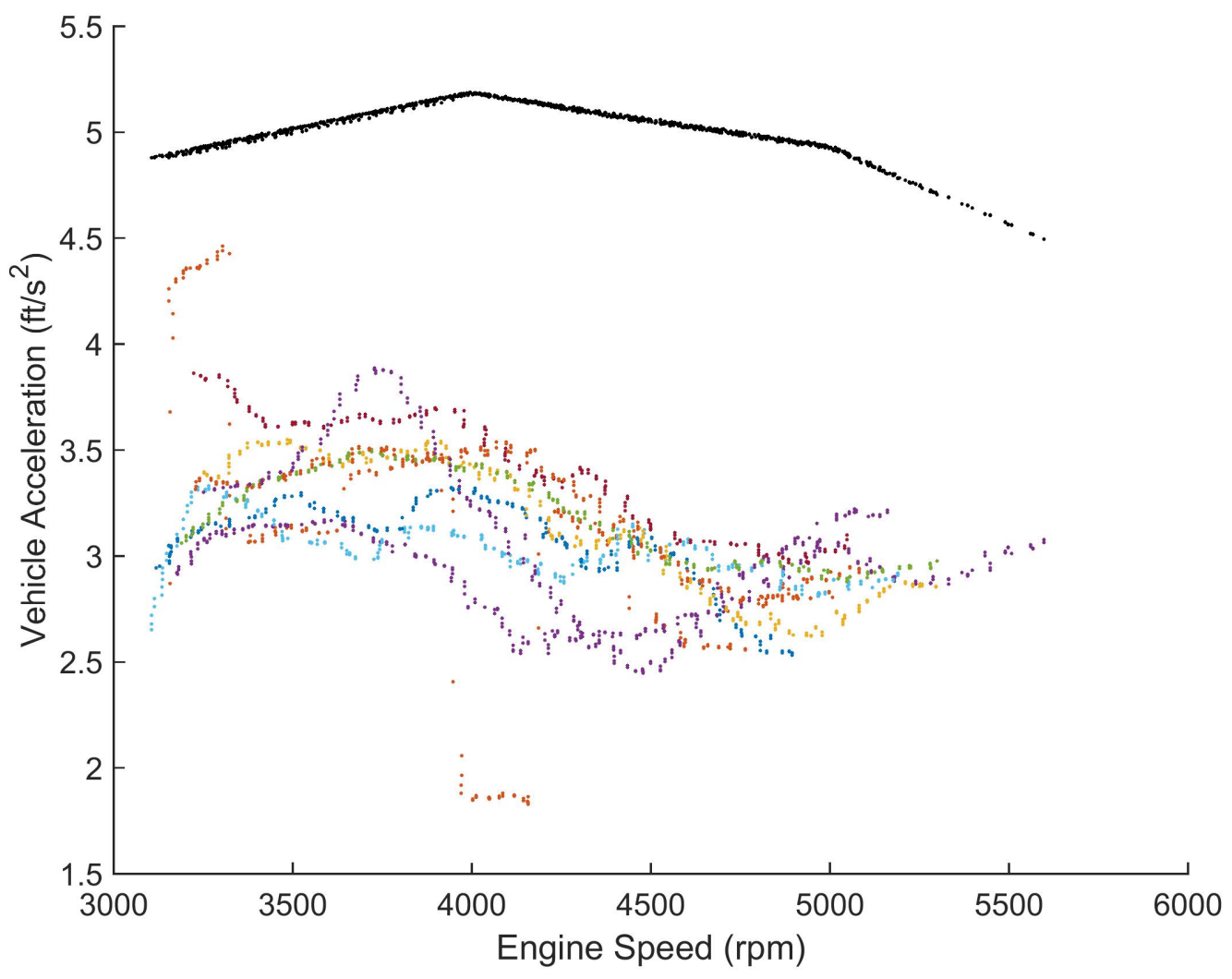

Figure 23: Vehicle Acceleration versus Engine Speed

Notice that both the theoretical and experimental accelerations follow the same trend, but the experimental acceleration seems to be a constant fraction of the theoretical. The drivetrain efficiency can be calculated at every point using the following equation: 


$$
\gamma=1-\frac{\dot{v}_{t h}-\dot{v}_{e x}}{\dot{v}_{t h}}
$$

Where:

$\dot{v}_{e x}$ is the experimental vehicle acceleration

The result can be seen in Figure 24.

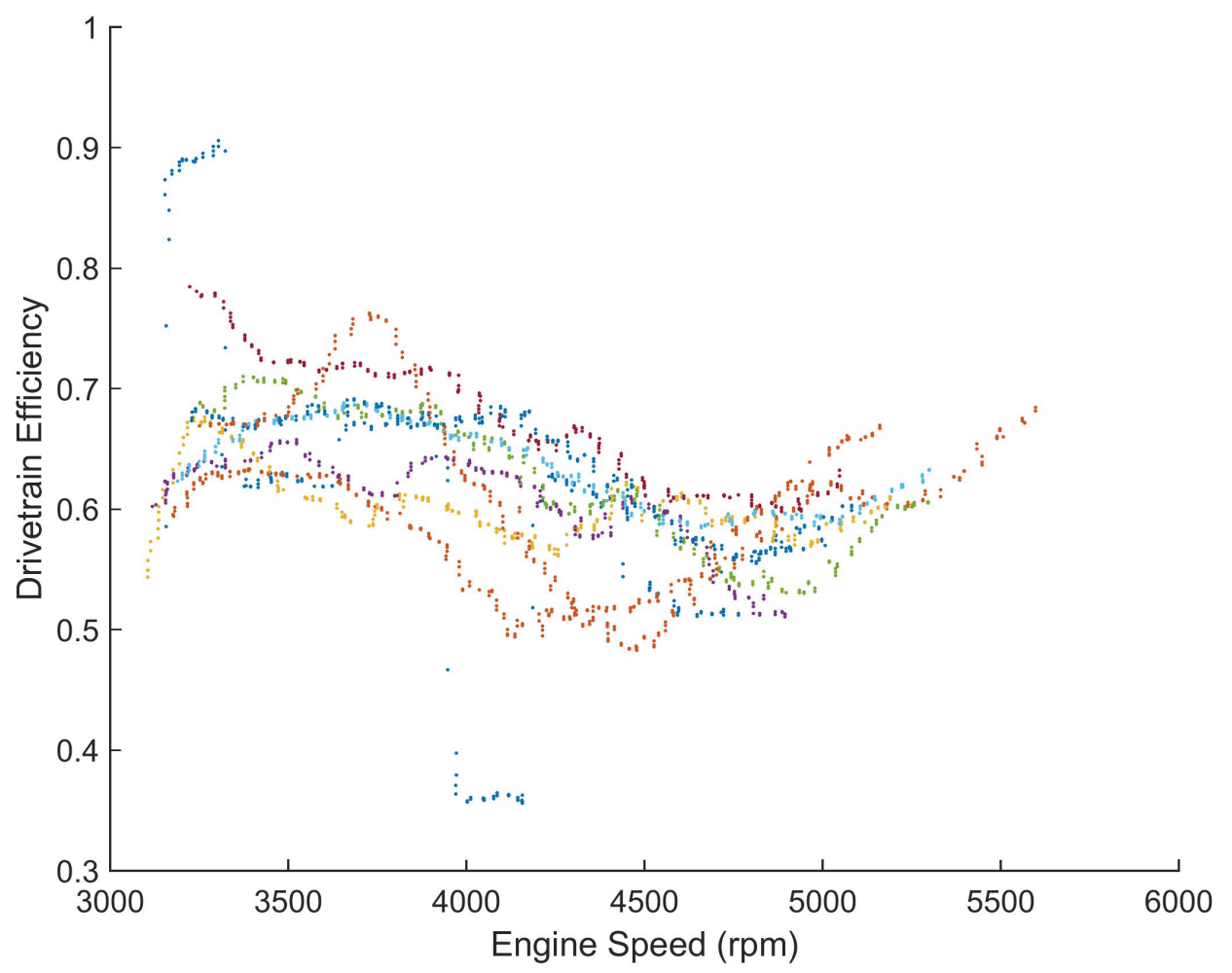

Figure 24: Drivetrain Efficiency versus Engine Speed

$\gamma$ is relatively constant, so the average of all efficiencies will be used as the drivetrain efficiency. This average is $62.1 \%$. 


\subsection{Clutch Torque Curve}

The next step is to find the unknown coefficients of the clutch torque equation (10), $C_{c}$ and $\omega^{\prime}$. Torque from the drivetrain can be calculated experimentally using Equation 26 (derived from Equation 3):

$$
\tau_{c}\left(\omega_{e}\right)=\frac{\dot{v}_{e x} m_{v} r}{\eta \gamma}
$$

A $\gamma$ of 0.621 will be used, as calculated from the previous section. Once $\tau_{c}\left(\omega_{e}\right)$ has been determined over a range of engine speeds, polynomial regression can be used to fit the data to a second-order polynomial. Figure 25 shows the experimental clutch torque versus engine speed from various accelerations. 


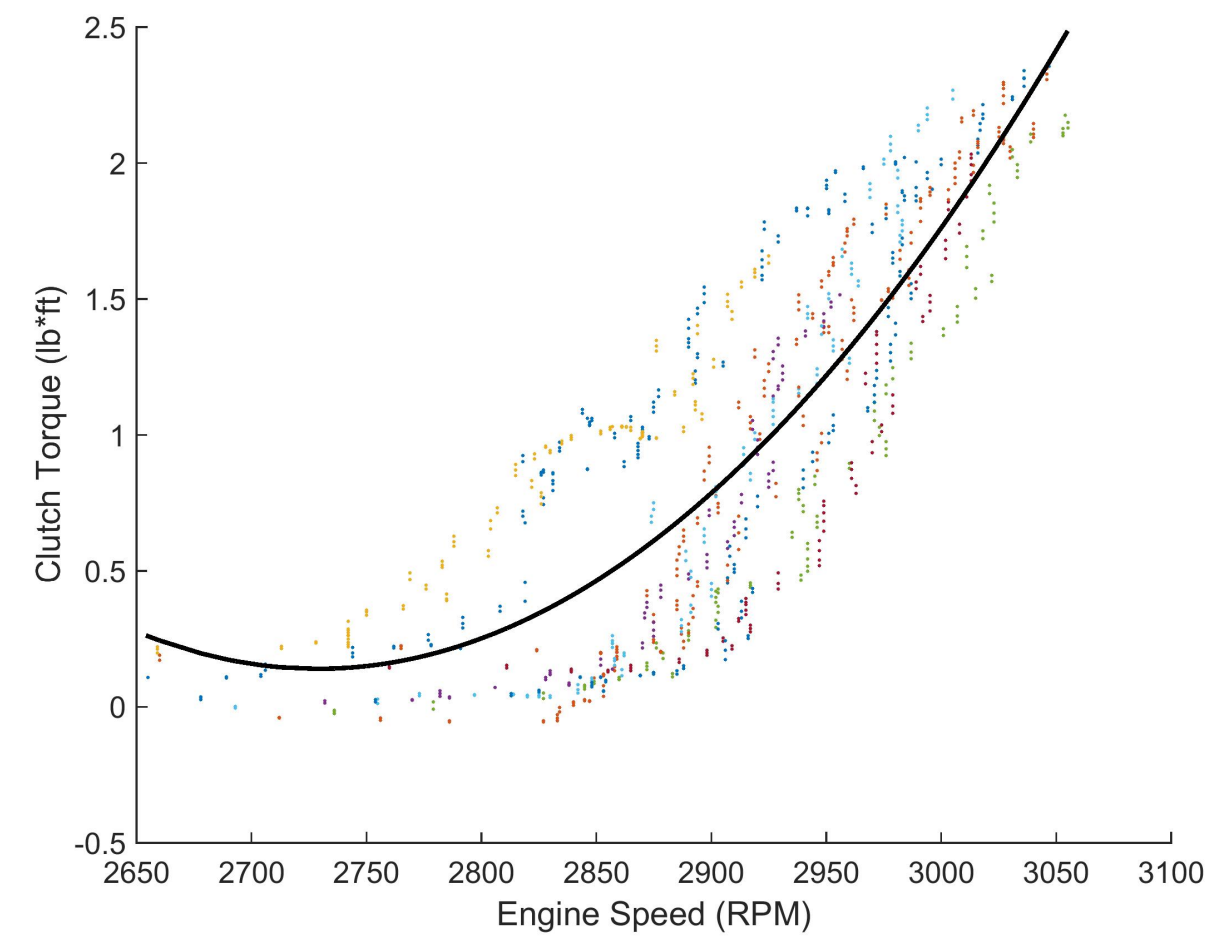

Figure 25: Clutch Torque versus Engine Speed

The black line in the center of the data is the least-squares polynomial. The coefficients of that polynomial can be used to calculate $C_{c}$ and $\omega^{\prime}$. The engagement speed of clutch, $\omega^{\prime}$, is $2730 \mathrm{rpm}$, and the clutch engagement coefficient, $C_{c}$, is $0.0020 \mathrm{lbf} /(\mathrm{rad} / \mathrm{s})^{2}$.

\subsection{Starting Penalties}

The final coefficients to determine are $K_{\omega}$ and $K_{m_{f}}$, the average engine acceleration and fuel flow rate when the engine is below idle speed. The engine idle speed is about $1000 \mathrm{rpm}$. In order to analyze the starting motor dynamics, the motor will be considered "starting" when the engine speed is above 0 but below $1000 \mathrm{rpm}$. 
Additionally, the motor will still be "starting" if it reaches $1000 \mathrm{rpm}$ but does not stay above it.

Figure 26 shows the total fuel consumption of a typical testing run. The red circles are the points at which the engine is "starting." Notice that the engine does not take long to start, and little fuel is consumed.

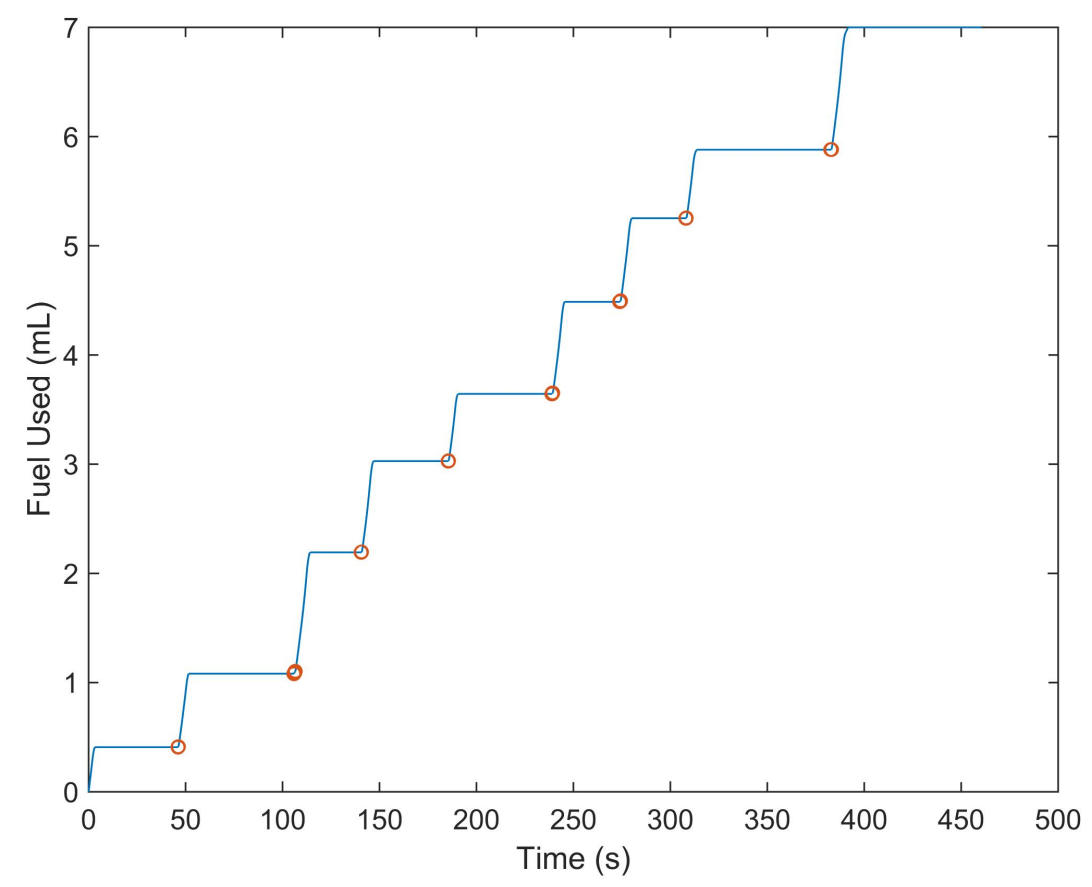

Figure 26: Total Fuel Consumption versus Time

Figure 27 shows the engine coolant temperature over time. Again, the red circles are when the engine is starting. The engine begins the testing run fairly cold but steadily warms up with every burn. 


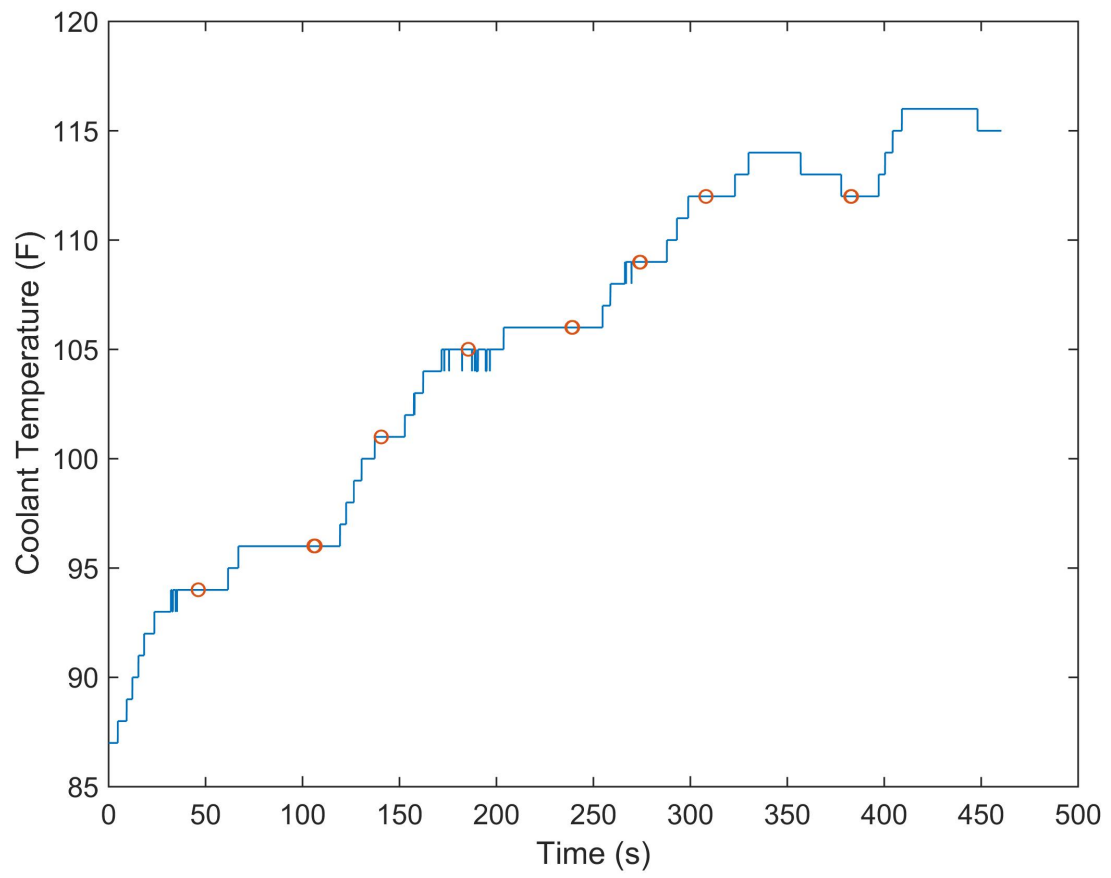

Figure 27: Engine Coolant Temperature versus Time

Figure 28 shows the time it takes for the engine to complete starting versus the start index. There were 8 starts analyzed, so there are only 8 data points in the figure. 


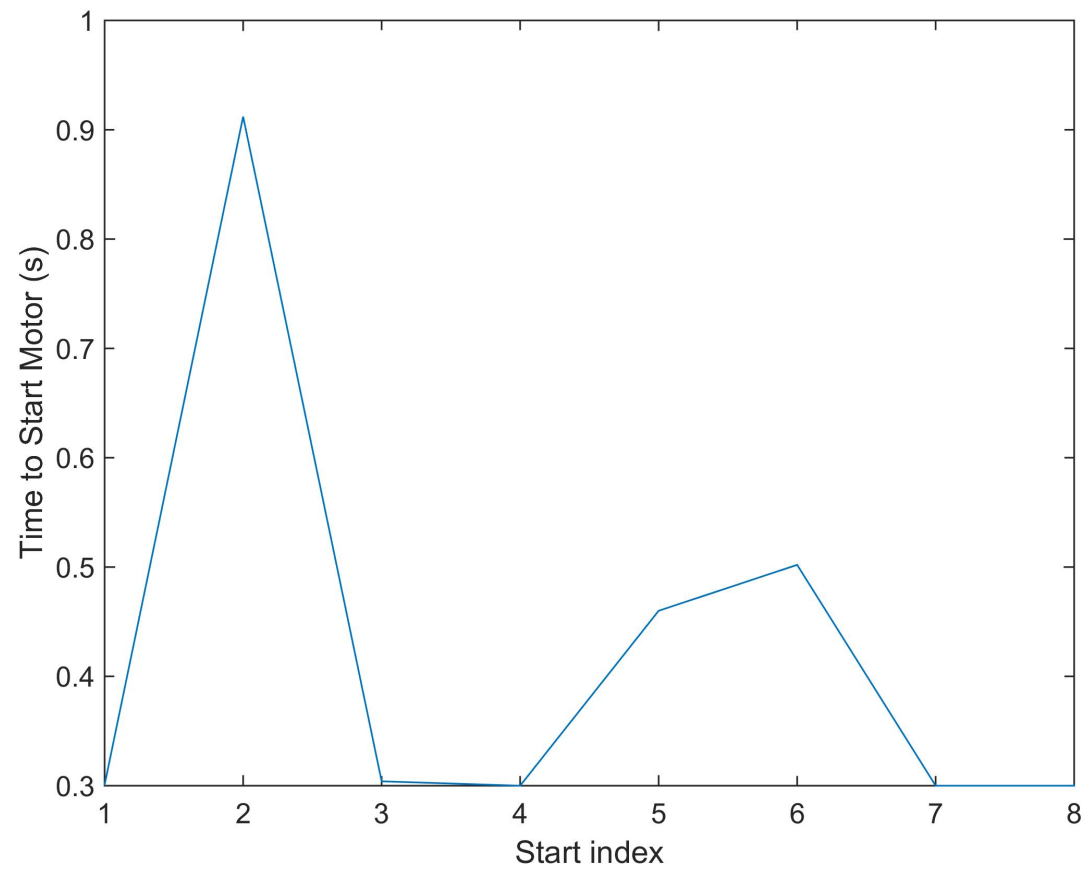

Figure 28: Time Taken to Start Motor

Figure 29 shows the total fuel consumed over each start. Notice that engine starting time and fuel consumed are relatively constant. It is expected that the engine would start faster and use less fuel as it warms up, but that is not clear from the data. Therefore, the assumption that the starting constants are not affected by temperature seems reasonable. 


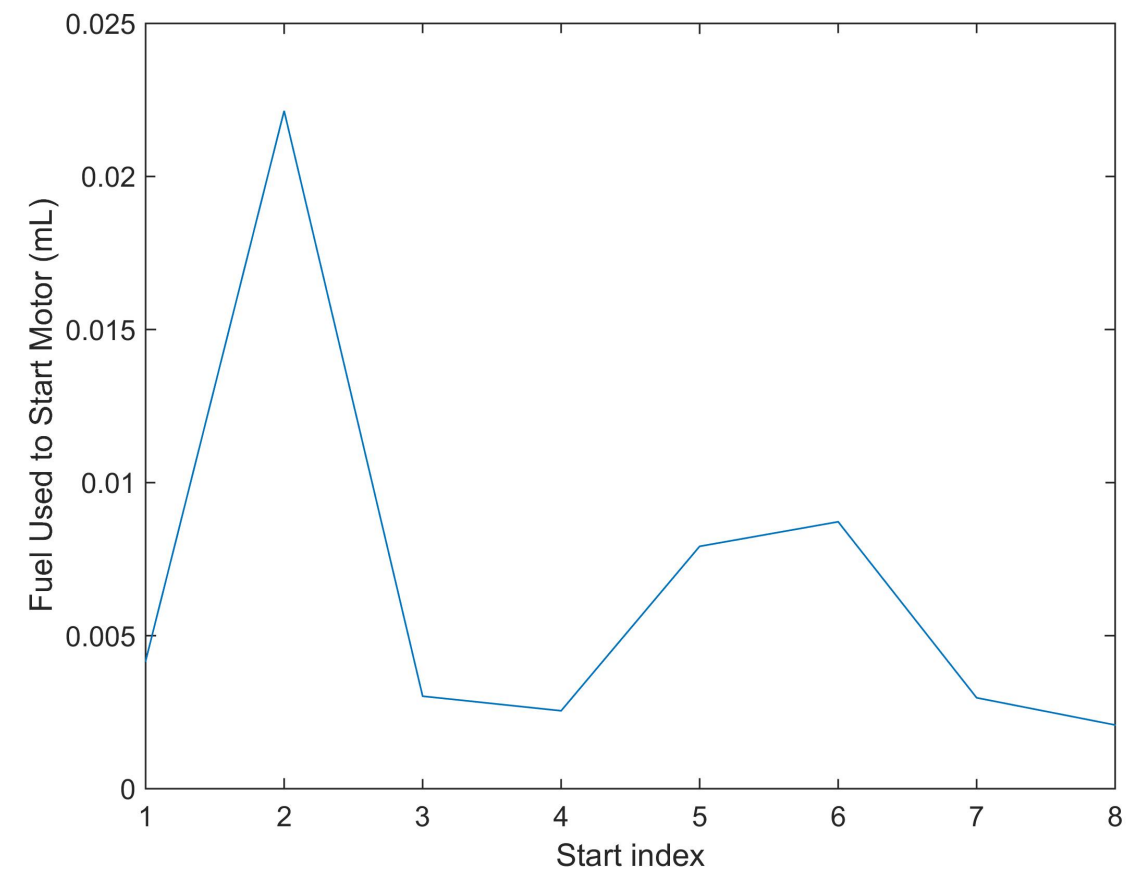

Figure 29: Fuel Consumed per Start

The average of the starting times is 0.42 seconds, while the average fuel consumed is $0.0067 \mathrm{~mL}$. The average fuel consumption divided by the average time determines the average starting fuel flow rate, which is equal to $0.0159 \mathrm{~mL} / \mathrm{s}$. The starting engine acceleration can be calculated by dividing the engine idle speed, $104.72 \mathrm{rad} / \mathrm{s}$, by the average starting time to get $247.92 \mathrm{rad} / \mathrm{s}^{2}$.

\subsection{Verifying the Simulation}

Now that all of the required coefficients have been determined, the simulation can be executed. Since testing data is only available from uneven tracks, and the exact slopes along the track are unknown, the track will be assumed to be flat in the simulation for this section. 
Figure 30 shows the measured vehicle speed in blue and the simulation output speed in red. Notice that the simulation output is perfectly consistent, while the actual speed varies significantly. Part of the difference is driver error, and part is the uneven track.

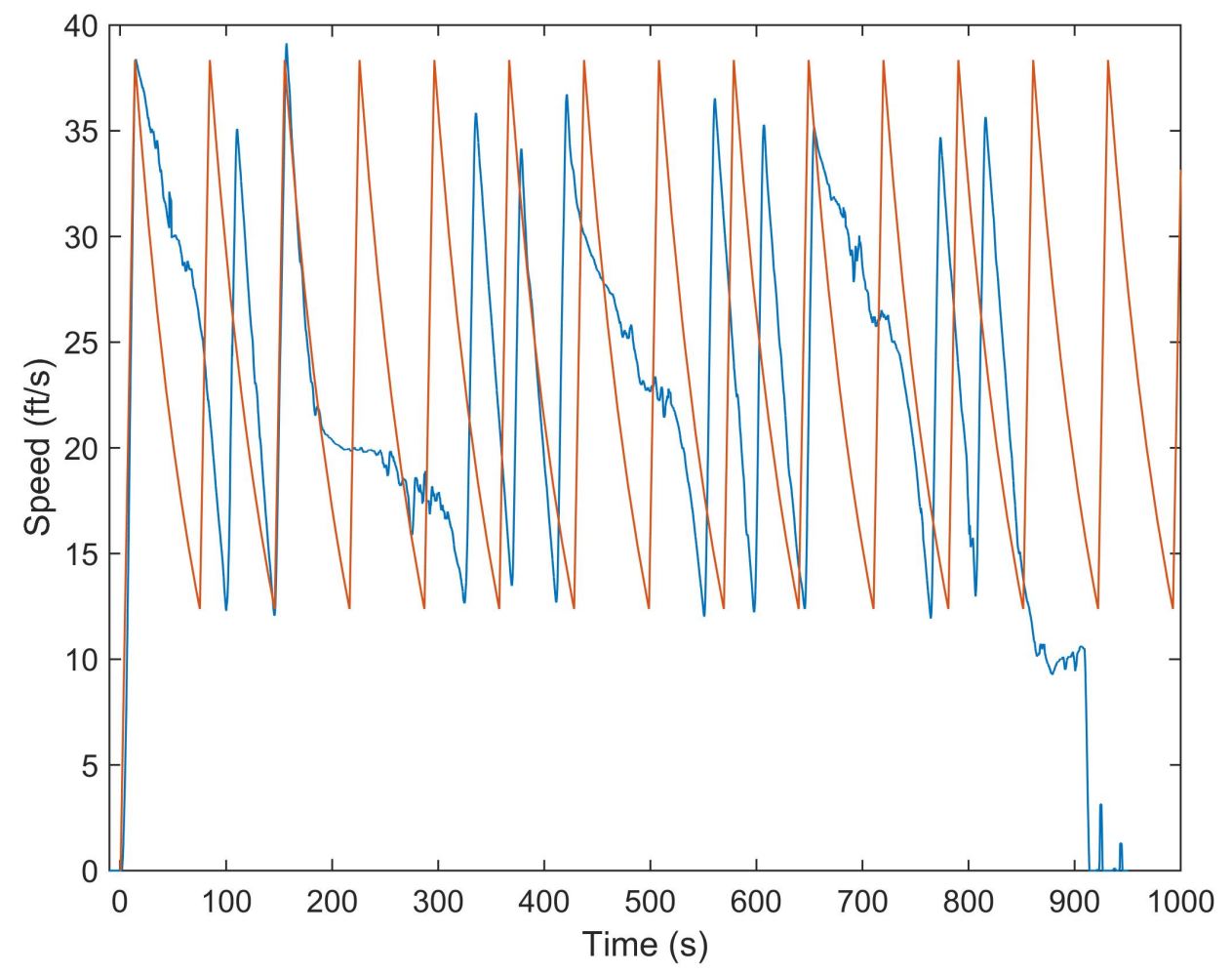

Figure 30: Vehicle Speed versus Time

Figure 31 shows a zoomed-in portion of Figure 30. The simulation output matches the actual speed fairly well, but the track unevenness makes it difficult to compare well. 


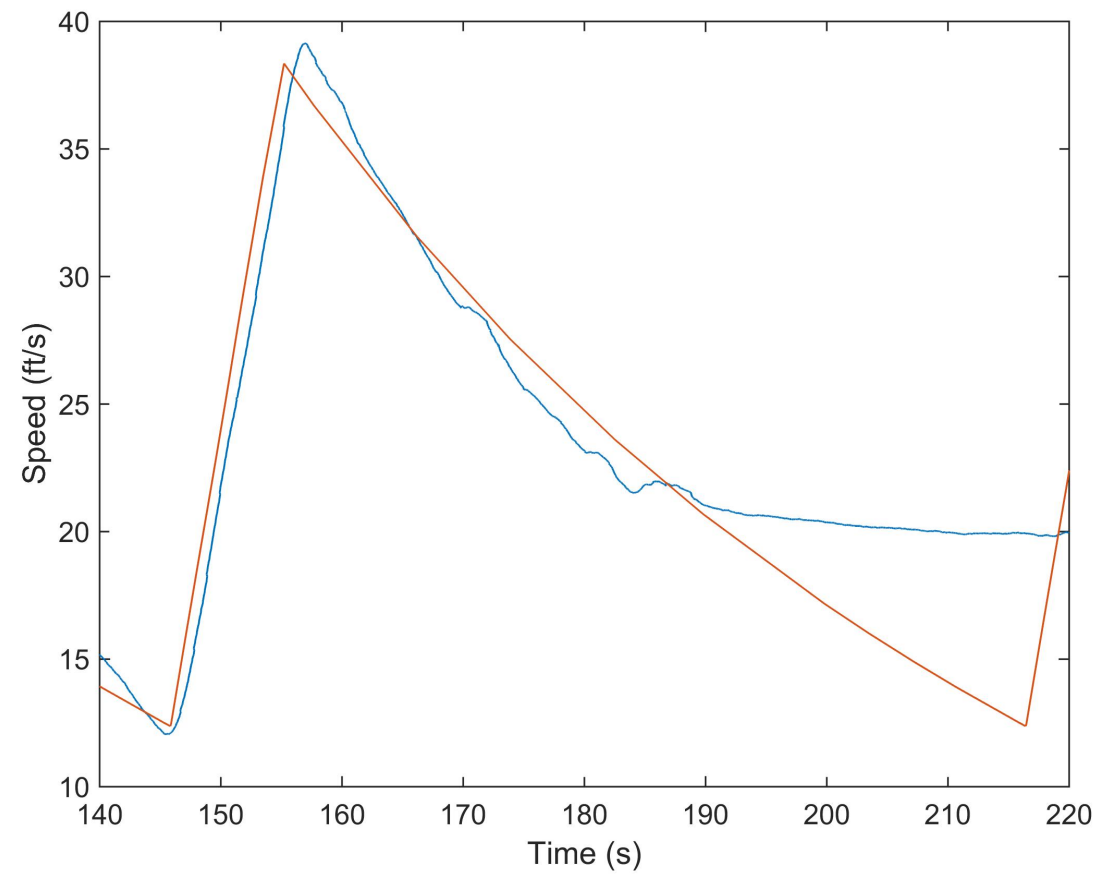

Figure 31: Zoomed In Vehicle Speed versus Time

Figure 32 shows the engine speed from the same testing data and simulation output. The actual engine speed is in blue, while the simulation output is in red. 


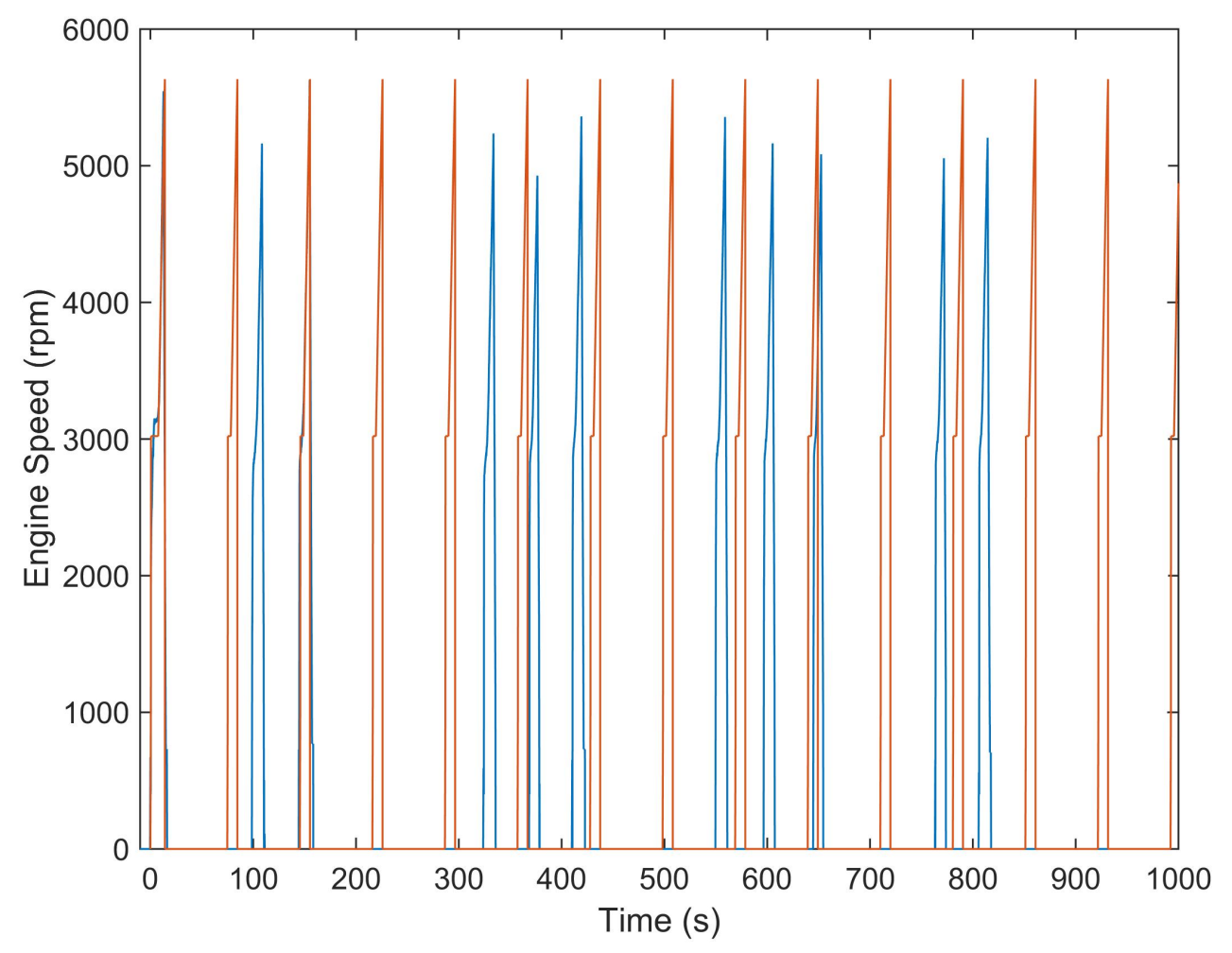

Figure 32: Engine Speed versus Time

Figure 33 shows a zoomed-in portion of Figure 32. Notice that the simulated engine speed matches fairly closely to the experimental engine speed. However, there are 2 main differences. The clutch simulation does not capture the exact dynamics of the system, but it is close. Additionally, the engine speed drops nearly instantly in the simulation, while it takes a small amount of time in the actual vehicle. 


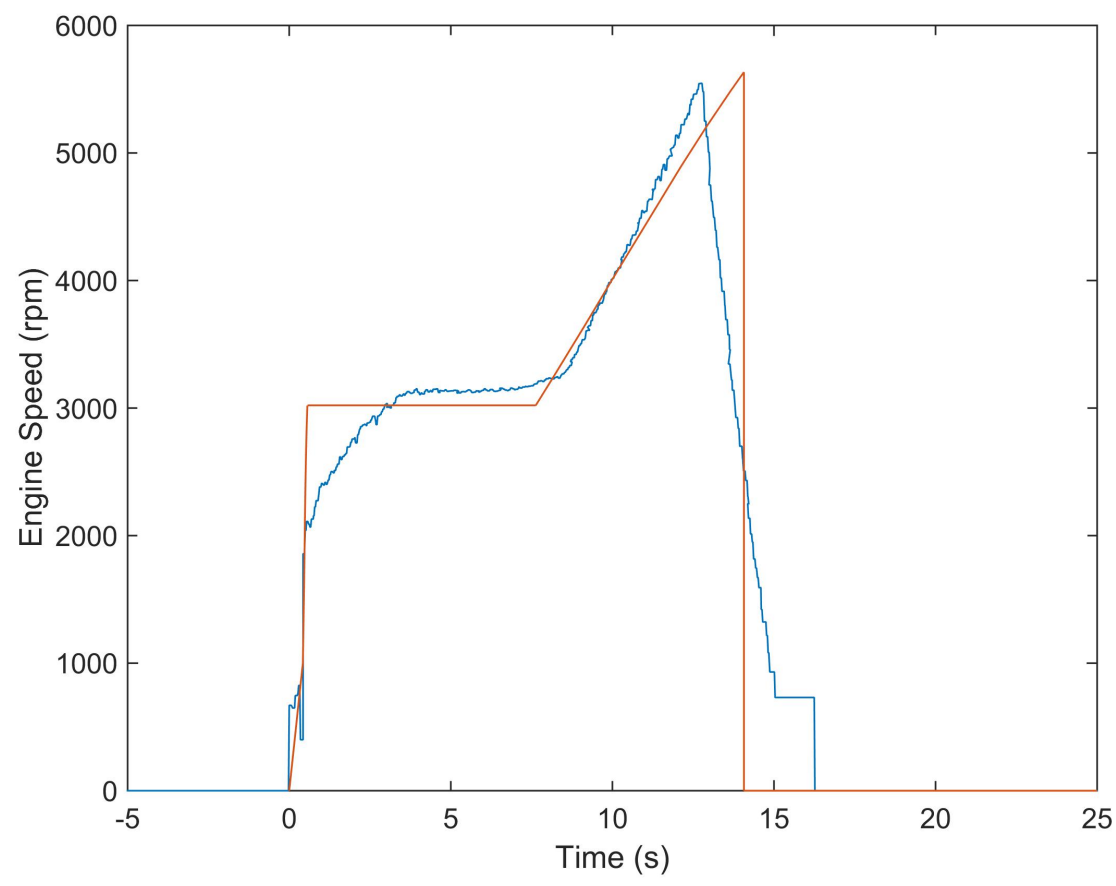

Figure 33: Zoomed In Engine Speed versus Time

Figure 34 shows the total fuel consumed over distance. 


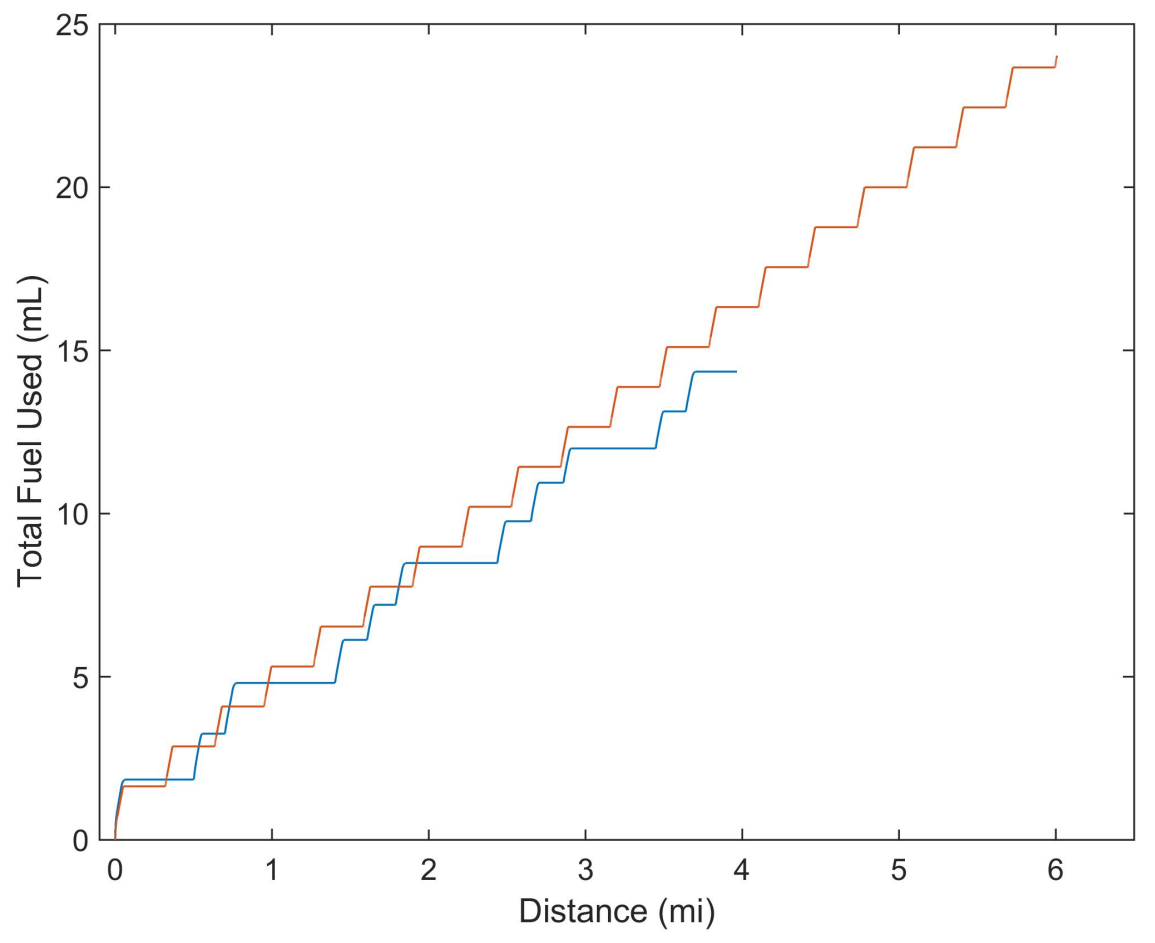

Figure 34: Total Fuel Consumption versus Distance

The fuel consumption does not exactly align, and this is likely because of 2 reasons: The engine takes a small amount of time to reach 0 rpm in reality, and the road load equation is slightly inaccurate. If the road load equation was accurate, the energy taken from the vehicle should be the same in the actual testing and simulation. This would mean that the slope of the total fuel consumption plot would be the same for experimental and simulated. Since there are 3 terms in the road load equation, it is not possible to determine which term is not accurate.

Overall, the simulation seems to match the actual vehicle well. With better data and testing methods, the simulation should be able to match the actual vehicle output even better. 


\section{Vehicle Parameter Analysis}

In the following sections, various vehicle parameters will be analyzed using the simulation.

\subsection{User Interface}

In order to make trade studies easy, a simple user interface was developed using MATLAB's GUIDE[9] user interface development tool. A single window is used to alter any simulation parameters and run trade studies quickly. This window is shown in Figure 35. 


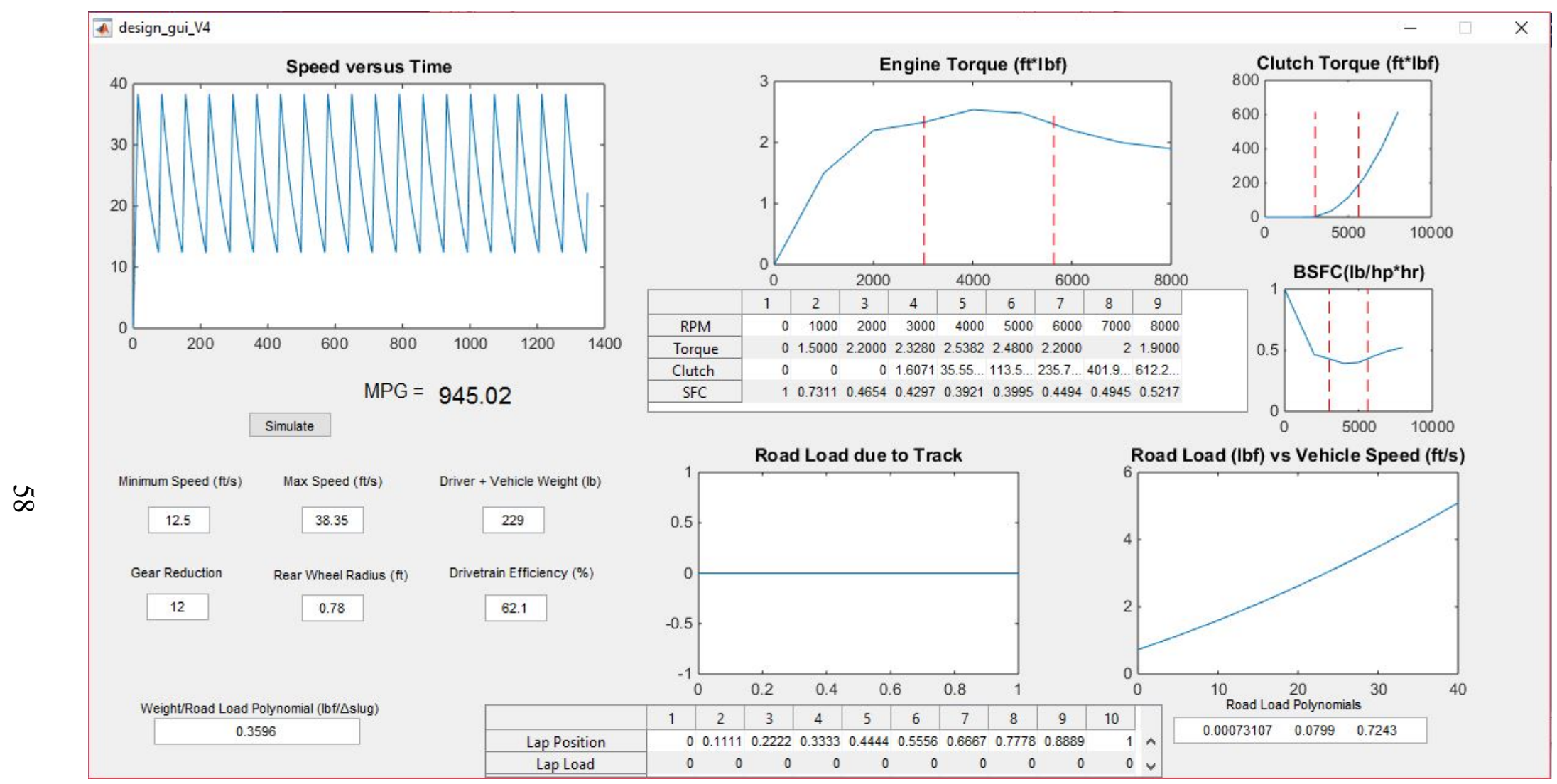

Figure 35: Simulation User Interface 
Detailed instructions to use the user interface can be found in the appendices.

\subsection{Vehicle Weight Analysis}

Historically, one of the most important parameters for vehicle efficiency has been weight. Every Shell Eco-marathon team tries to minimize weight to maximize fuel efficiency. Using the simulation, the effect of weight on fuel efficiency can be determined. Every parameter remains constant except road load, which changes by Equation 22. The output is shown in Figure 36.

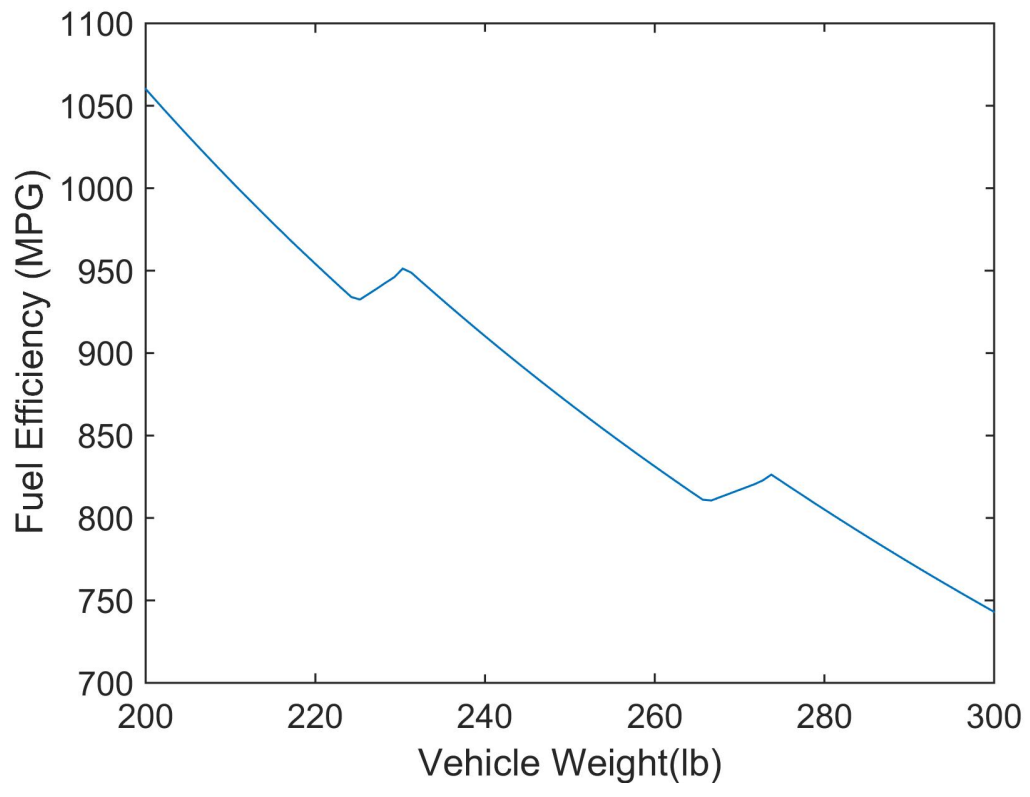

Figure 36: Fuel Efficiency versus Weight

Overall, there is a downward trend from increasing total vehicle weight, which is not surprising. There are slight increases in fuel efficiency at certain weights; these are not numerical errors. As vehicle weight increases, the total number of coasts and burns is reduced. This is due to the increased vehicle inertia, which 
demands longer burns and coasts to speed up and slow down respectively. When the time spent coasting and burning is changed, the vehicle ends the competition run either burning or coasting. If the vehicle ends the competition run coasting, the fuel efficiency is better than if energy was wasted burning at the very end. That is why there are slight increases at certain weights.

\subsection{Optimizing Vehicle Speed and Gear Ratio}

The minimum and maximum speeds of the vehicle may have a dramatic effect on fuel economy, since they directly affect the operating range of the engine. The optimal minimum and maximum speeds could be found by keeping every other parameter constant and finding the maximum vehicle efficiency. However, the final drive ratio should also be allowed to change at the same time, since it also changes the operating range of the engine. Therefore, for the remainder of this section, the simulation output will be considered a function of 3 variables:

$$
M P G=f\left(v_{\min }, v_{\max }, \eta\right)
$$

Where:

$v_{\text {min }}$ is the minimum vehicle speed $(f t / s)$

$v_{\text {max }}$ is the maximum vehicle speed $(\mathrm{ft} / \mathrm{s})$

MATLAB will be used to maximize fuel efficiency. However, its optimization functions are set to minimize, not maximize. Therefore, the optimization goal will be to minimize negative fuel efficiency. If the simulation time output is greater than 24 minutes (the time allotted for a competition run), the resulting fuel econ- 
omy is not valid. Therefore, the function will return "NaN", or Not-a-Number[11]. Equation 28 shows the optimization function:

$$
f_{\text {opt }}\left(v_{\text {min }}, v_{\text {max }}, \eta\right)=\left\{\begin{array}{ll}
-M P G\left(v_{\text {min }}, v_{\text {max }}, \eta\right) & t_{\text {final }} \leq 24 \min \\
N a N & t_{\text {final }}>24 \text { min }
\end{array}\right\}
$$

There are 2 main options available for global minimization in MATLAB: GlobalSearch and MultiStart. GlobalSearch was chosen since it runs more efficiently than MultiStart on one processor[10], and it is unknown how many processors will be available to any user running the optimization.

The GlobalSearch algorithm essentially works by evaluating the optimization function at a starting point and then finding a local minimum from that point[19]. This repeats for many different starting points to find all the local minima. The lowest minimum is then the global minimum. Ideally, each starting point should lead to a different local minimum. This can be accomplished by accurately finding the radius of the function's basin of attraction. The radius is the distance in solution space from the starting point to the local minimum. If the radius is too large, there may be local minima missed. If the radius is too small, the same local minima will be found repeatedly. The radius of the basin of attraction is estimated as part of the GlobalSearch algorithm[19], but MATLAB allows the user to specify a distance threshold factor. This factor is multiplied by the estimated basin radius to get a more conservative radius if the threshold is smaller than 1.

Additionally, a user can specify how many trial points will be tested. If a trial point is close to the previously found global minimum, the local minimum from that 
point will be found. For this reason, it is expected to have a more accurate result if many trial points are used. On average, a simulation run takes about 0.37 seconds to complete, so many trial points will take a very long time to go through. Each GlobalSearch was ran with a random starting point to ensure that the true global minimum was found. The results of the optimization are summarized in Table 1.

\begin{tabular}{||cccc||}
\hline Fuel Economy (MPG) & Speed $(f t / s)$ & Final Drive Ratio & Simulation Runs \\
\hline \hline 1146.2 & $17.92-26.86$ & 15.17 & 13152 \\
\hline 1146.0 & $17.36-27.55$ & 14.77 & 7800 \\
\hline 1137.3 & $17.55-27.41$ & 16.23 & 3197 \\
\hline 1115.2 & $16.96-28.69$ & 16.44 & 3082 \\
\hline
\end{tabular}

Table 1: Optimization Results

Notice that the maximum fuel economy was found when more runs were simulated. However, after a certain amount of simulation runs, there is not much of an advantage to looking at more trial points. The first GlobalSearch ran the simulation 13,152 times, and it found a maximum fuel economy of 1146.2 MPG. The second search found a maximum of 1146.0 MPG, which is only 0.2 MPG less. However it ran the simulation only 7,800 times.

An interesting way to visualize the GlobalSearch optimization is to plot all the various points that the algorithm tries. Since $v_{\min }, v_{\max }, \eta$, and $M P G$ make up a fourth dimensional solution space, it is more practical to show the trial points on a second dimensional plot. Figure 37 shows a scatter plot of each trial point projected onto the $M P G-\eta$ axes. 


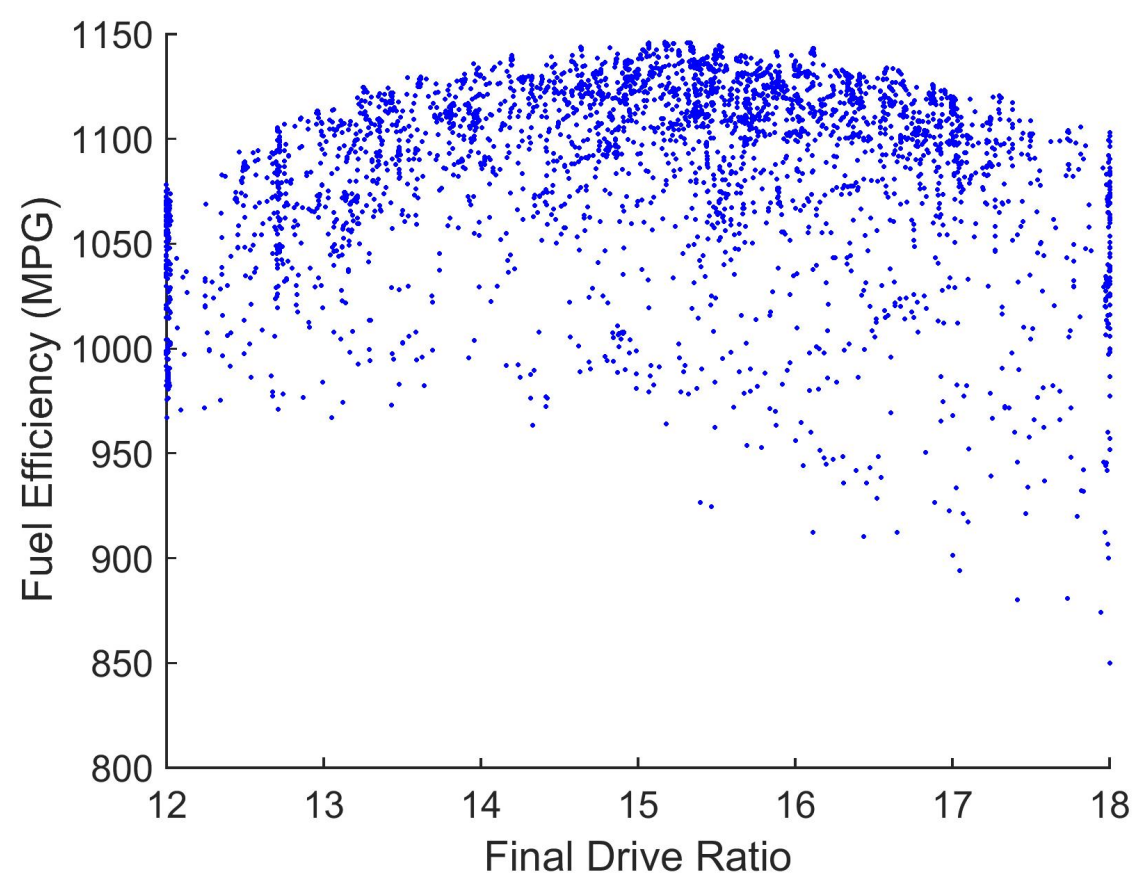

Figure 37: Fuel Efficiency versus Gear Ratio, Varying Vehicle Speed

There is a large variety in fuel economy when speed is changed, meaning that improper vehicle speeds can severely impact fuel economy. The lowest efficiency is about $850 M P G$, while the highest is $1146.2 M P G$. Figure 37 was generated using the search from the top of Table 1. Even though there were 13,152 simulation runs, there are not that many points on the scatter plot because many points do not complete the competition in time.

All the searches came up with reasonably close results, so the global maximum is likely the one shown at the top of Table 1 . If it is not the true global maximum, it is likely very close.

The first way to verify the global maximum is to plot fuel economy versus final drive ratio while keeping $v_{\min }$ and $v_{\max }$ constant. The values of $v_{\min }$ and $v_{\max }$ used 
are 17.92 and 26.86, the values from the top of Table 1. This is shown in Figure 38.

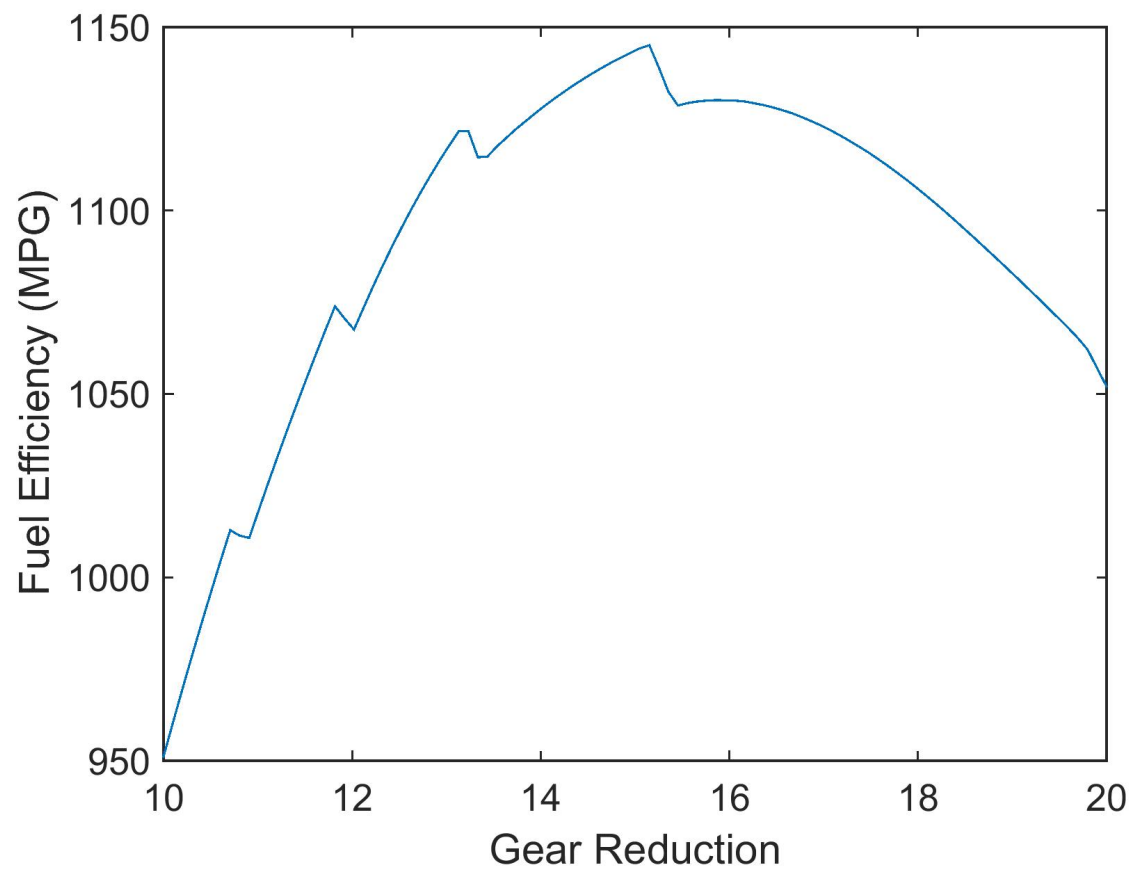

Figure 38: Fuel Efficiency versus Gear Ratio, Constant $v_{\min }$ and $v_{\max }$

The peak fuel economy is about $1145 M P G$ at 15.2 gear reduction, which verifies the maximum found through GlobalSearch. The jagged sections of the plot are caused by ending the simulation on a coast or a burn. Changing the final drive ratio slightly changes when the vehicle coasts and burns, and it is always more efficient to end on a coast.

The second way to verify the global maximum is to plot $M P G$ versus $v_{\min }$ and $v_{\max }$ in a 3-D surface plot. For the plot, final drive ratio is set to 15.17 , the optimal final drive ratio found at the top of Table 1. Three views of the 3-D plot will be shown. 


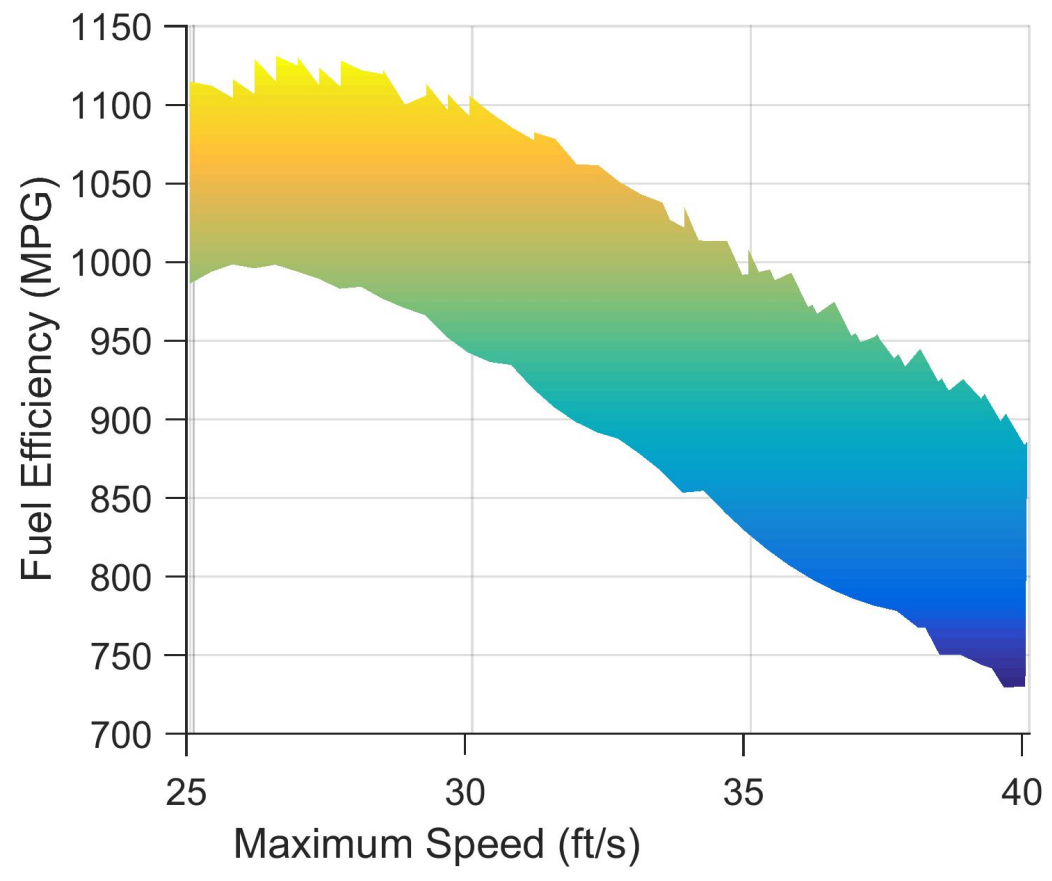

Figure 39: Fuel Efficiency versus Maximum Speed, Constant $\eta$

Figure 39 shows the relationship between maximum vehicle speed and fuel economy. The fuel efficiency decreases with top speed; this is a result of the higher engine speeds and higher road load losing more energy over time.

Figure 40 shows the relationship between minimum vehicle speed and fuel economy. There is not much of a distinct relationship as with maximum vehicle speed, but there is a clear maximum around $18 \mathrm{ft} / \mathrm{s}$. 


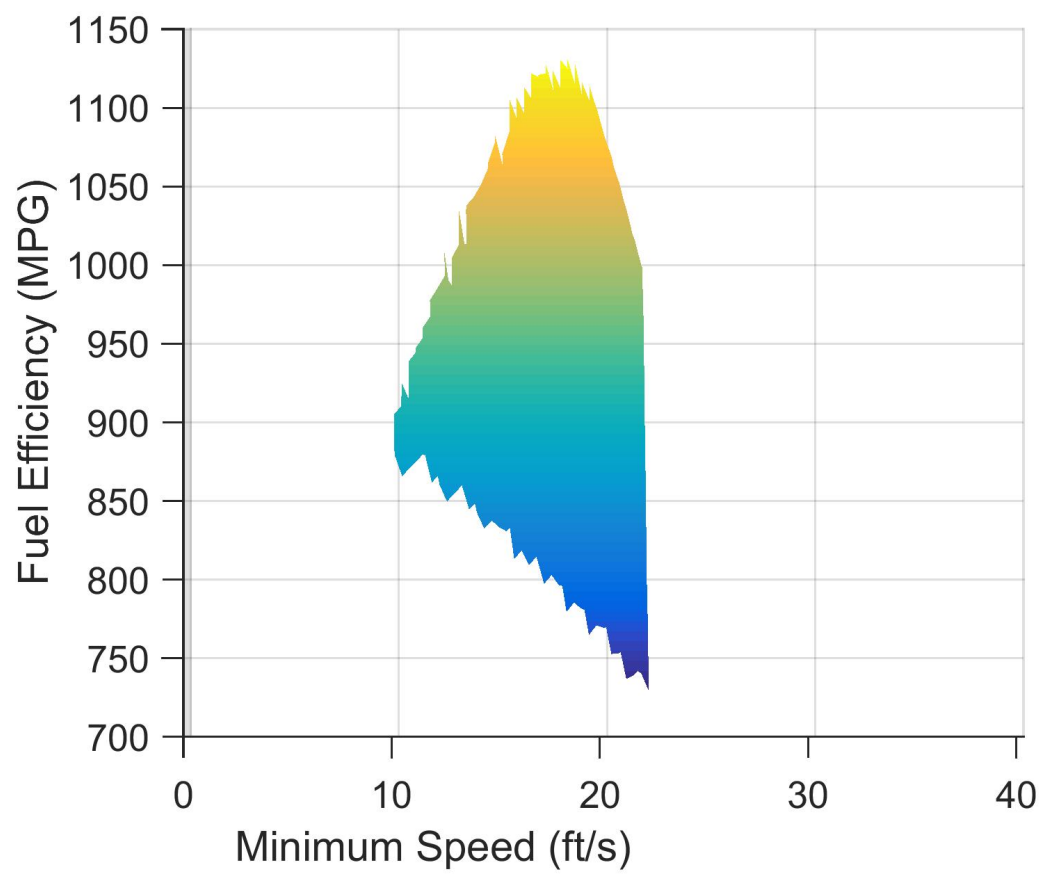

Figure 40: Fuel Efficiency versus Minimum Speed, Constant $\eta$

Figure 41 shows the relationship between maximum and minimum vehicle speed and fuel economy. 


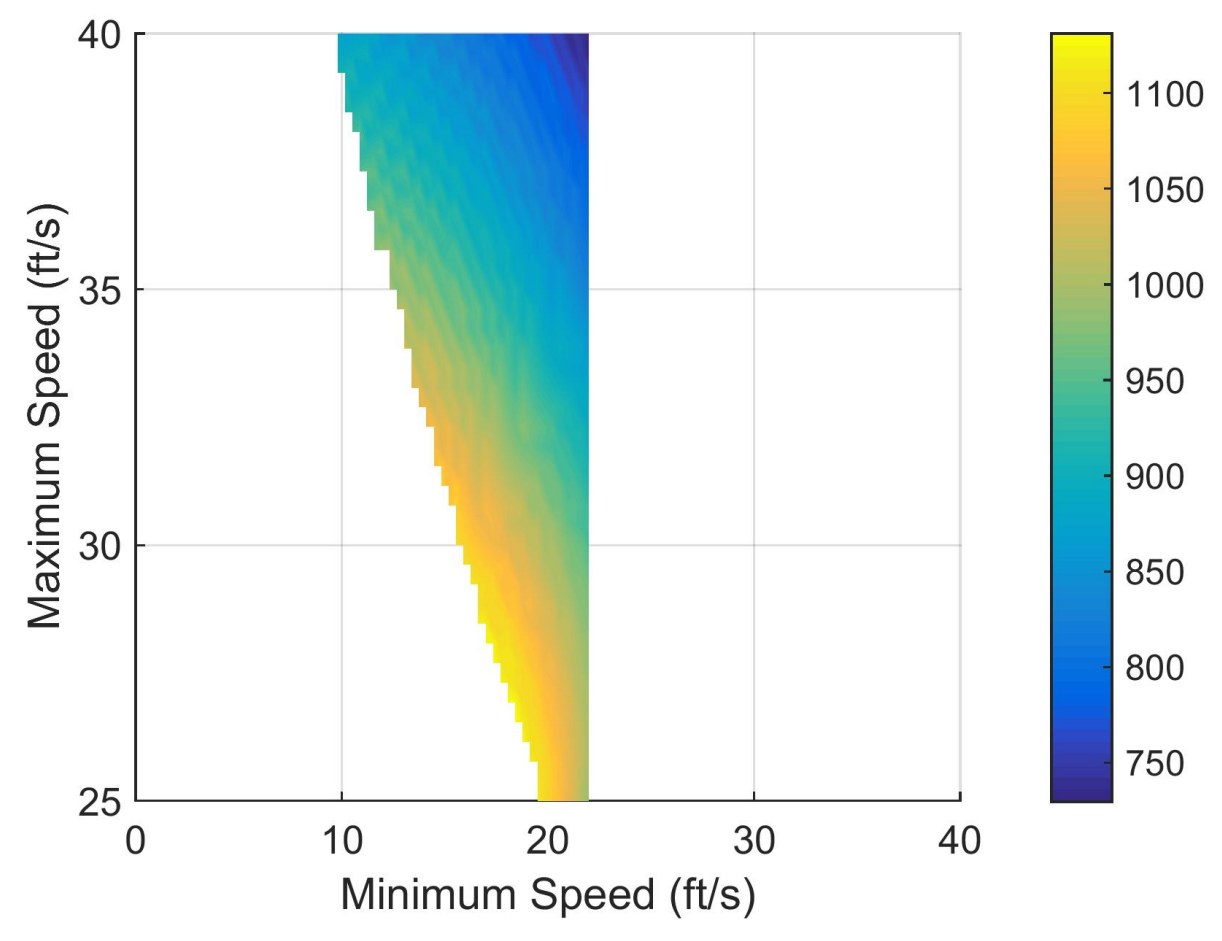

Figure 41: Fuel Efficiency versus Maximum and Minimum Speed, Constant $\eta$

The optimal minimum and maximum speeds are about 18 and 27 respectively, verifying the optimal speeds found by the GlobalSearch algorithm.

\subsection{Optimization Discussion}

Table 2 shows the difference between the optimized and unoptimized vehicles.

\begin{tabular}{||c|ccc||}
\hline & Fuel Economy (MPG) & Vehicle Speeds $(f t / s)$ & Final Drive Ratio \\
\hline \hline Optimized & 1146.2 & $17.92-26.86$ & 15.17 \\
\hline Unoptimized & 1055 & $12.5-38.35$ & 12 \\
\hline
\end{tabular}

Table 2: Optimization Comparison

In order to further verify the optimal speeds and final drive ratio, it is useful to 
look at how the vehicle operates under the optimal conditions. When the minimum vehicle speed is 17.92 , the clutch housing speed is $3329 \mathrm{rpm}$. This is slightly above $3021 \mathrm{rpm}$, the speed at which centrifugal clutch torque equals the torque of the engine. This essentially means that the clutch is never slipping when the vehicle is driven at optimal speeds. Since a slipping clutch loses energy, this makes sense to maximize efficiency. Figure 42 shows actual engine speed in blue and optimal simulated engine speed in red.

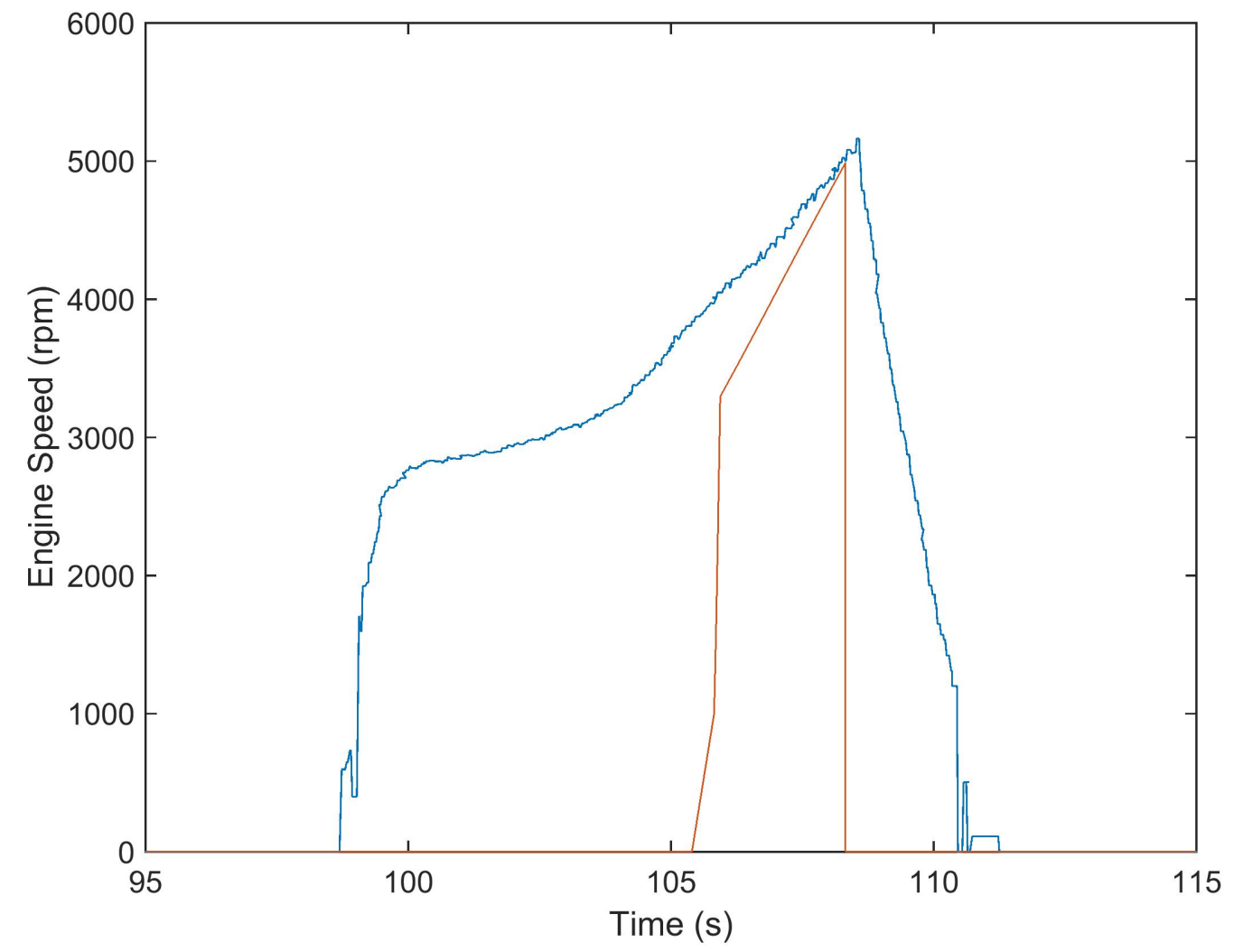

Figure 42: Engine Speed versus Time

Notice that the clutch does not slip at all in the optimal simulation, and the 
optimal burn time is about $30 \%$ of the original burn time. However, at optimal speeds, the vehicle must use the engine much more often. Figure 43 shows the actual vehicle speed in blue and the optimal simulation speed in red.

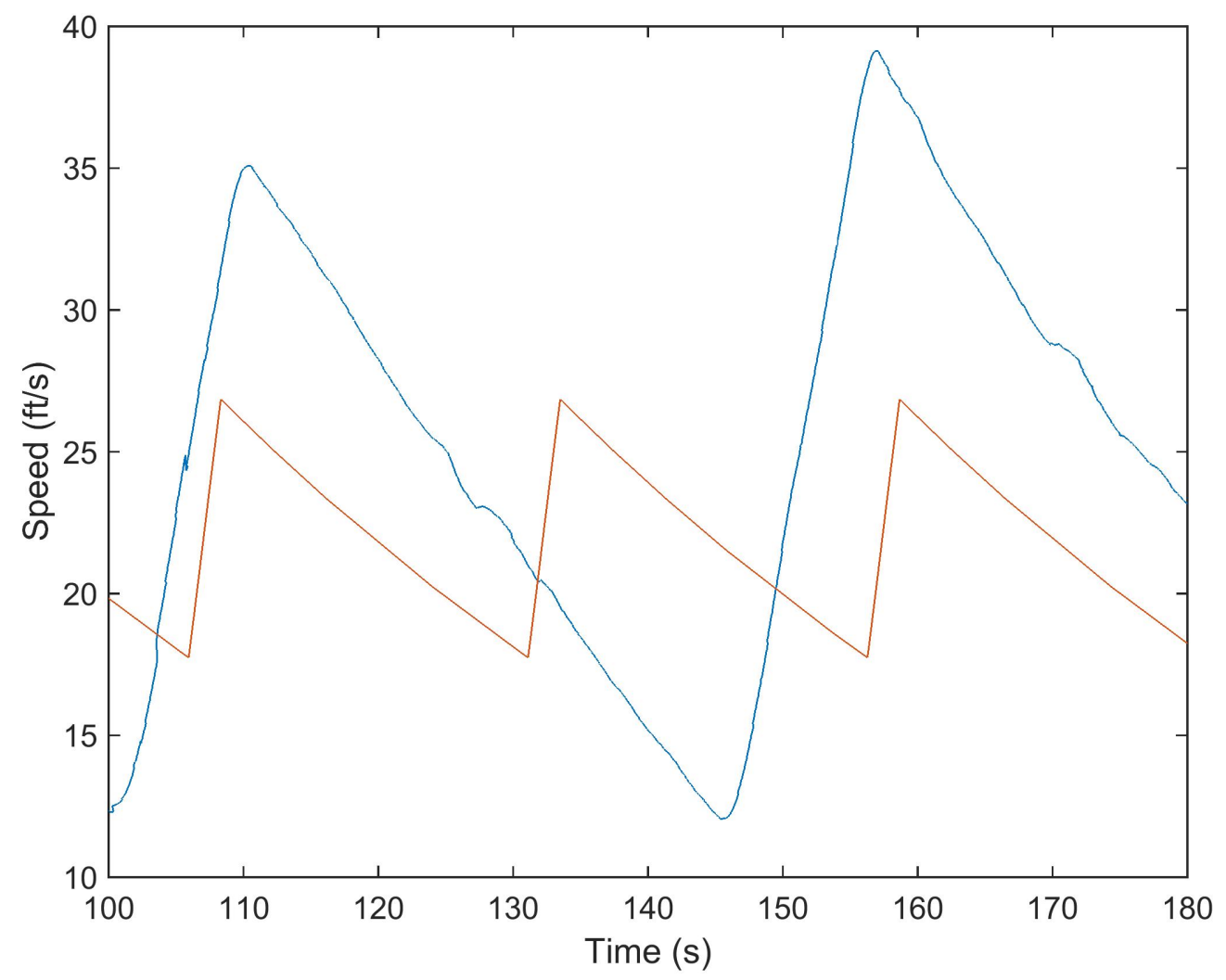

Figure 43: Vehicle Speed versus Time

The optimal top speed is the speed at which the vehicle can barely make it to the finish line in time. If the top speed is any lower, the vehicle will not finish. This makes sense, since faster speeds will increase road load and also move the peak operating engine speed higher. Both of those will increase the amount of energy wasted by the vehicle. Figure 44 shows the actual vehicle fuel consumption in blue 
and the optimal fuel consumption in red.

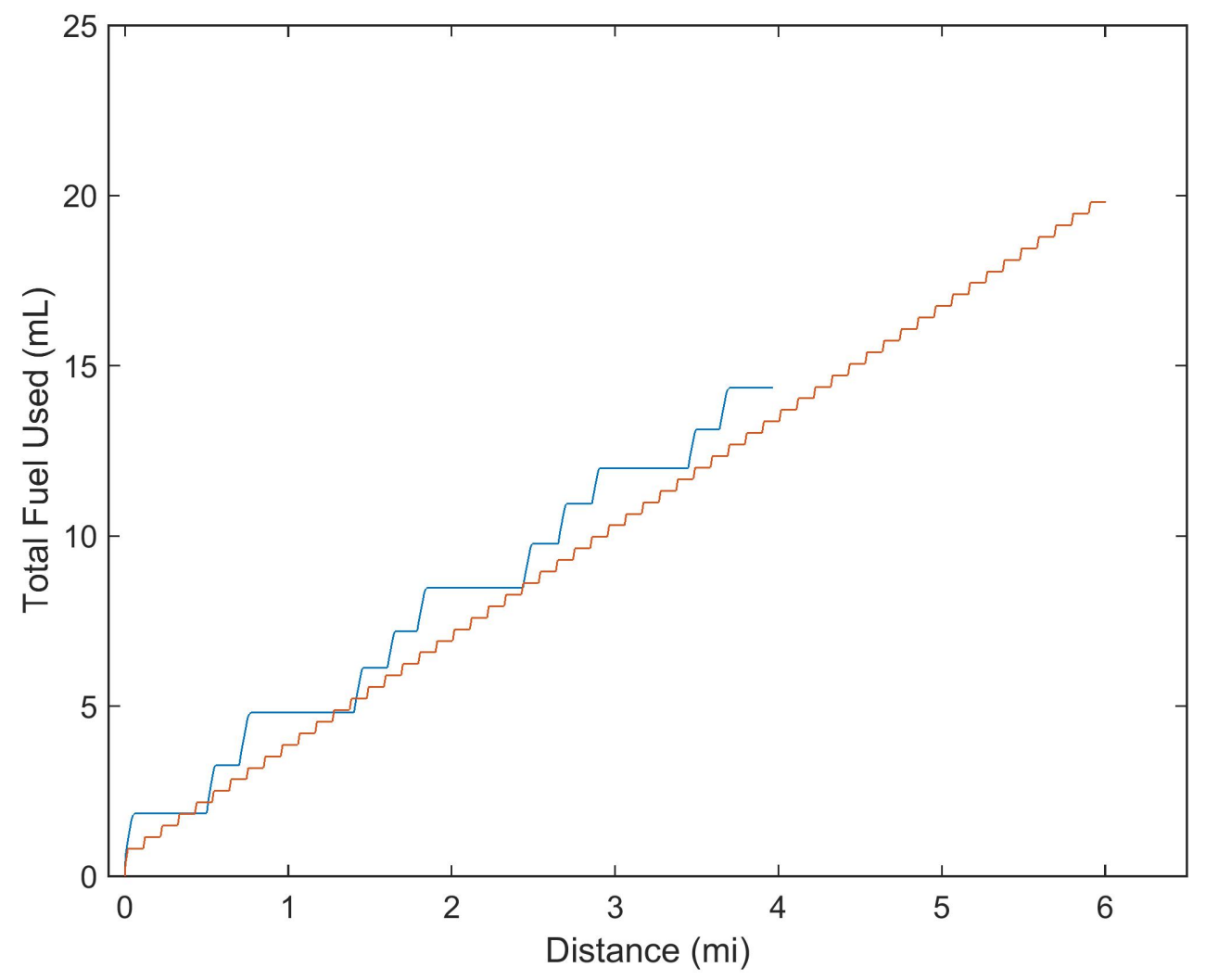

Figure 44: Fuel Consumption versus Distance

During testing, the vehicle ran at 1055.0 $M P G$. The optimal vehicle runs at 1146.2 $M P G$, which is an $8.6 \%$ increase in fuel efficiency. 


\section{Conclusion}

Overall, the optimization study will guide future Cal Poly Supermileage teams to better fuel economy. However, there are some shortfalls that must be addressed in the future.

The calculated road load coefficients have considerable uncertainty. A rearwheel Hall effect sensor should be installed to accurately measure vehicle speed. A pitot tube may also be mounted to the car to negate any wind effects from the aerodynamic drag calculation. Additionally, a more accurate way to measure the slope of the test track must be found.

The greatest inaccuracy from the simulation comes from the engine turning off. In the simulation, the engine dies instantly, while the actual engine takes a few seconds to actually reach $0 \mathrm{rpm}$. In the future, it may be useful to tweak the simulation equations (15 and 16) to account for the engine shutoff dynamics.

The optimization study heavily relies on accurate starting penalties. A more accurate model would take into account how much energy is consumed by the starter motor to start the engine every time. This additional penalty would likely increase the speed difference between the optimal minimum and maximum speeds, reducing the total number of burns required.

In general, a review of all the data analysis in this study, with new data would be added, would be useful to validate the results and ensure accuracy. All the MATLAB code utilized to analyze the data is easy to use, so this should be possible in the future.

From this study, a few recommendations can be asserted. Driving strategy can 
be changed quickly, so the user interface should be used as a tool to ensure that the driver always drives most efficiently, no matter what vehicle is driven. In the future, a higher final drive reduction (15.2 from 12) should be used to maximize fuel economy. The user interface should also be used to determine what engine tune is most efficient. For every tune, the only information needed is new torque and BSFC curves; then a simulation can be performed. The analysis and user interface presented in this study should help guide future Supermileage teams, and hopefully it will continue to demonstrate how every vehicle can achieve the ultimate goal of higher fuel efficiency. 


\section{REFERENCES}

[1] William L. Brogan. Modern Control Theory. Prentice Hall, Upper Saddle River, New Jersey, 1991.

[2] Pedro De Figueiredo Vieira Carvalheira. Simulation of the performance of an extra-low fuel consumption vehicle. Proceedings of the IASTED International Conference on Modeling and Simulation, 15(1):424-429, 2004.

[3] Karol Cichonski, Katarzyna Jezierska-Krupa, Marcin Glen, and Wojciech Skarka. The comparative study of drivetrain of high-performance electric vehicle. Diagnostyka, 15(2):65-70, 2014.

[4] Thomas D. Gillespie. Fundamentals of Vehicle Dynamics. Society of Automotive Engineers Inc, Warrendale, Pennsylvania, 1992.

[5] Eric J. Griess. Benchmarking, characterization and tuning of shell ecomarathon prototype powertrain. 2015.

[6] Sophie Jan. Minimizing the fuel consumption of a vehicle from the shell ecomarathon: A numerical study. ESAIM - Control, Optimisation and Calculus of Variations, 19(2):516-532, 2013.

[7] Autosport Labs. Racecapture/pro. https://www autosportlabs. com/racecapturepro-2/. Accessed: 2/19/2017. 
[8] T. Manrique, M. Fiacchini, T. Chambrion, and G. Millerioux. Mpc for a low consumption electric vehicle with time-varying constraints. IFAC Symposium on System Structure and Control, 5(1):833-838, 2013.

[9] MathWorks. Create apps with graphical user interfaces in matlab. https : / / wWw. mathworks.com/discovery/matlab-gui.html. Accessed: $2 / 27 / 2017$

[10] MathWorks. How globalsearch and multistart work. https://www. mathworks.com/help/gads/ how-globalsearch-and-multistart-work.html. Accessed: $2 / 28 / 2017$

[11] MathWorks. Not-a-number. https://www.mathworks.com/help/ matlab/ref/nan.html. Accessed: 2/28/2017.

[12] Muhammad Rizuwan Mustaffa, Wan Ahmad Najmi B. Wan Mohamed, and Rahim B Atan. Analytical approach to predict hydrogen consumption of a lightweight pem fuel cell vehicle. IEEE International Conference on Control System, Computing and Engineering, pages 489-494, 2013.

[13] J.-C Wasselynck Olivier, G Trichet, D Bernard, N Hmam, S Chevalier, S Josset, C Auvity, B Squadrito, and Gaetano. Multiphysics modeling and driving strategy optimization of an urban-concept vehicle. IEEE Vehicle Power and Propulsion Conference, 2015.

[14] S. S. Rattan. Theory of Machines. McGraw-Hill, 2 Pennsylvania Plaza, New York City, New York, 2014. 
[15] Y. Saboohi and H. Farzaneh. Model for optimizing energy efficiency through controlling speed and gear ratio. Energy Efficiency, 1(1):65-76, 2008.

[16] J.J. Santin, C.H. Onder, and J. Bernard. The World's Most Fuel Efficient Vehicle, Design and Development of Pac-car II. ETH Zürich, Zürich/Singen, Switzerland, 2007.

[17] Shell. Shell eco-marathon 2017 rules. http://www.shell. com/energy-and-innovation/shell-ecomarathon/ for-participants/tech-tips-and-tricks/

_jcr_content/par/expandablelist/

expandablesection_603560196.stream/1472744744388/

7b204129356de26d47c9f21e2c8f58e07f3e2e4f266ae7714c8e6b17bb7051e1/ sem-2017-rules-chapter-1.pdf. Accessed: 3/2/2017.

[18] U.S. Geological Survey. Performance specification digital terrain elevation data. https://dds.cr.usgs.gov/srtm/version2_1/ Documentation/MIL-PDF-89020B.pdf. Accessed: 2/20/2017.

[19] Zsolt Ugray, Leon Lasdon, John C. Plummer, Fred Glover, James Kelly, and Rafael Martí. Scatter search and local nlp solvers: A multistart framework for global optimization. INFORMS Journal on Computing, 19(3):328-340, 2007. 


\section{APPENDICES}

\section{A. User Interface Instructions}

There are two parts to user interfaces in MATLAB: a .fig and .m file. The .fig file controls the layout of the user interface (the location of buttons, etc.), while the .m file contains the MATLAB code that runs in the background when a button is pushed on the user interface. To start the user interface, make sure the .fig and .m file are in the same folder. Then run "Design_GUI.m". The user interface will open and start with pre-filled in values. These default values can be changed by altering the values towards the top of the .m file.

To simulate a competition run with the starting values, click "Simulate". MATLAB figures will show up within a few seconds showing how the vehicle performed. Additionally, the vehicle's fuel efficiency in $M P G$ is shown next to the "Simulate" button. Any parameter in the user interface can be changed by clicking on the box and typing in a new number. As soon as "Simulate" is clicked again, the new numbers will be used for another simulation.

To perform a weight sensitivity analysis, type in 2 numbers separated by a space into the "Driver + Vehicle Weight (lb)" section, and then click "Simulate". 100 simulations will be performed from the first weight to the second weight, and a figure will automatically generate showing how fuel efficiency is affected by total vehicle weight. Note that this analysis strongly depends on the accuracy of the "Weight/Road Load Polynomial" parameter, which is located in the lower left-hand corner of the user interface. This parameter is discussed in section 4.3. 
To perform a final drive analysis, type in 2 numbers separated by a space into the "Gear Reduction" section, and then click "Simulate". 100 simulations will be performed from the first final drive ratio to the second, and a figure will automatically generate showing how fuel efficiency is affected by final drive reduction.

To perform an optimal speed analysis, type in 2 numbers into the Minimum and Maximum Speed sections, 4 numbers total, and then click "Simulate". 1600 simulations will be performed, so this takes significantly longer than the previous analyses. A 3D surface plot will be generated showing the relationship between minimum and maximum speeds and fuel efficiency.

To numerically optimize speed and final drive ratio using the GlobalSearch algorithm, type in 2 numbers into three sections: Minimum Speed, Maximum Speed, and Gear Reduction. Then click "Simulate" to begin the optimization. This will go through about 10,000 simulations, so expect to wait 30 minutes to an hour to get the result.

If the user interface layout needs to be changed, type in "guide" into the main MATLAB command line to bring up GUIDE, the tool used to build the interface. 


\section{B. MATLAB Code}

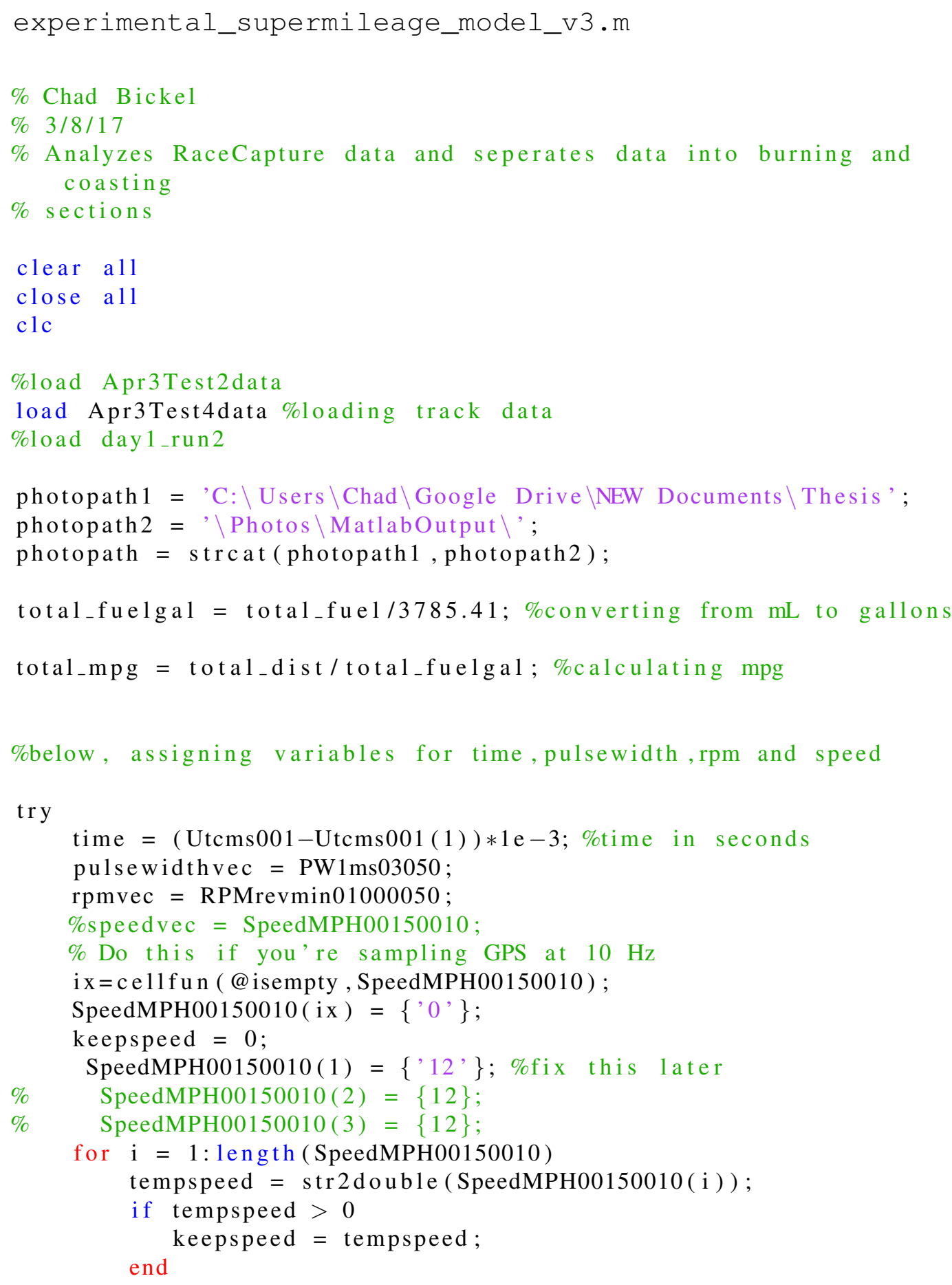




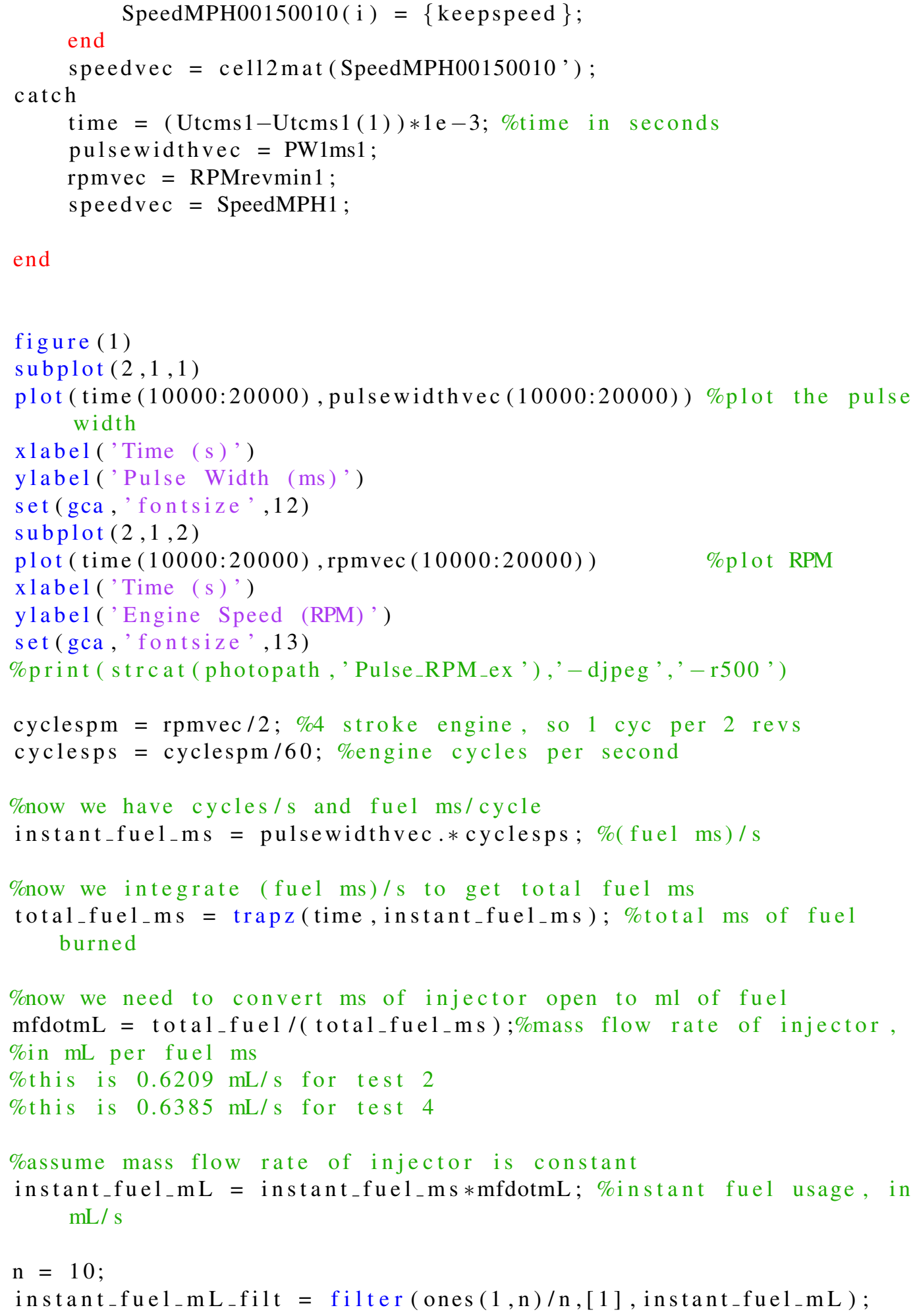




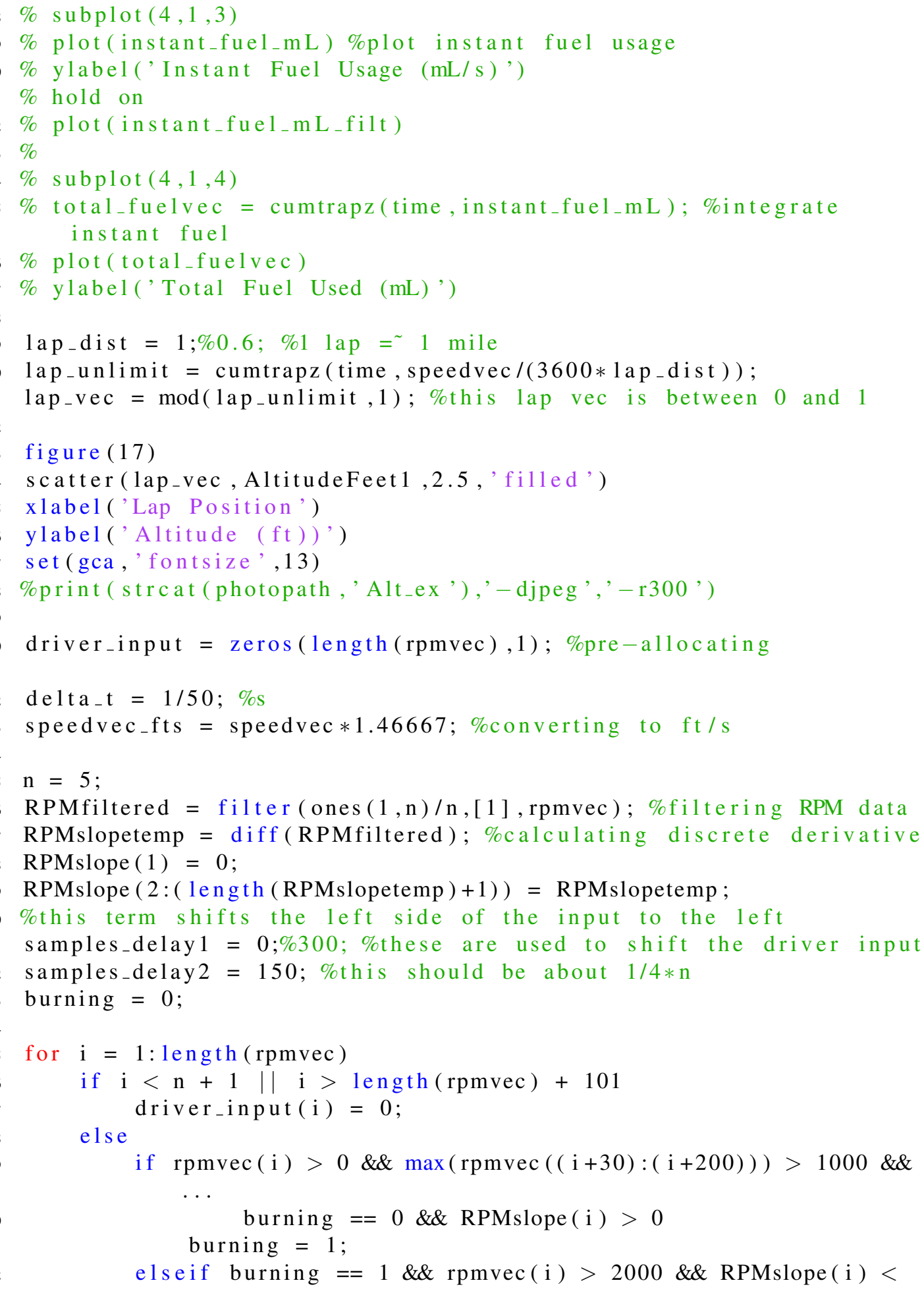




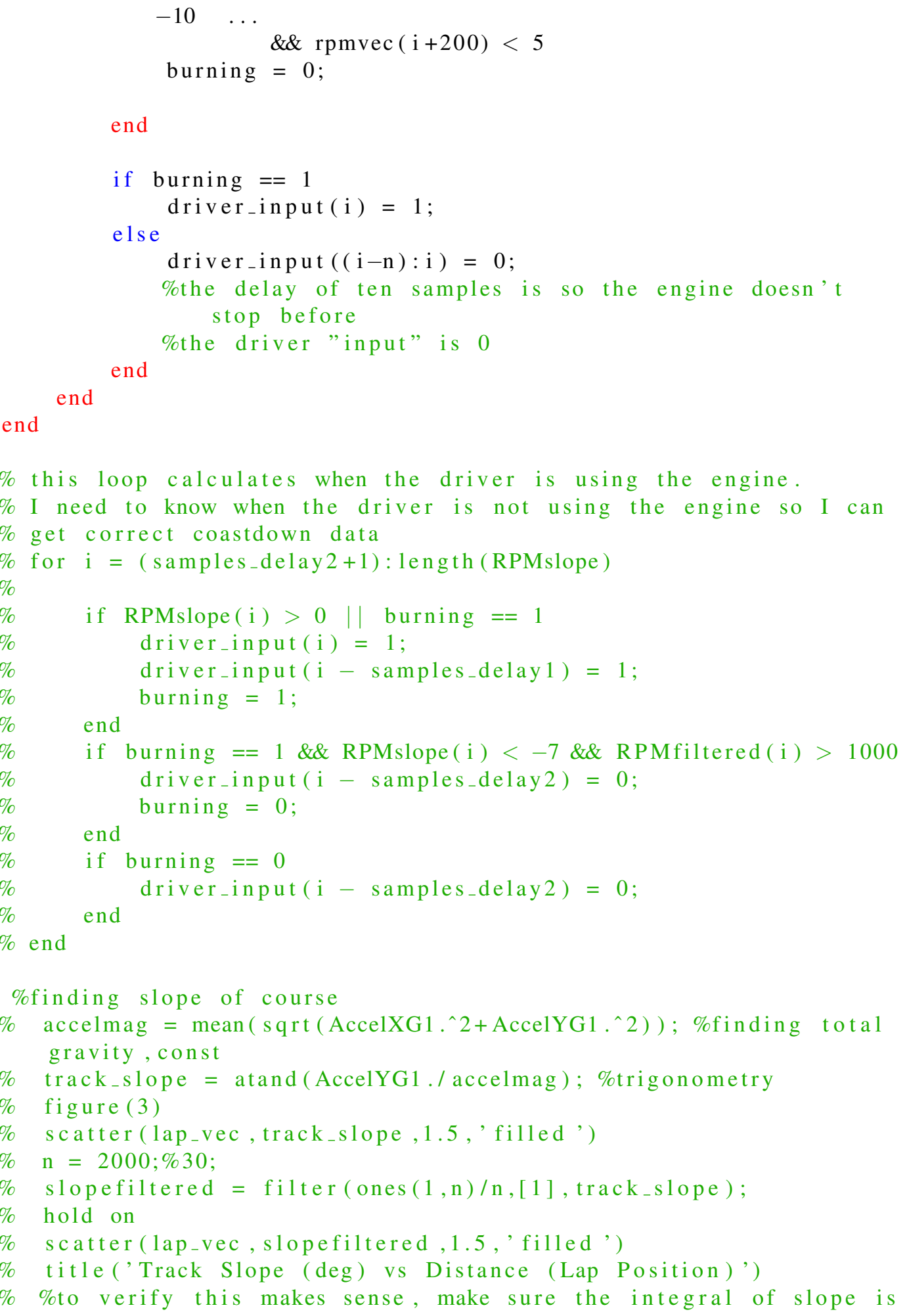

147 


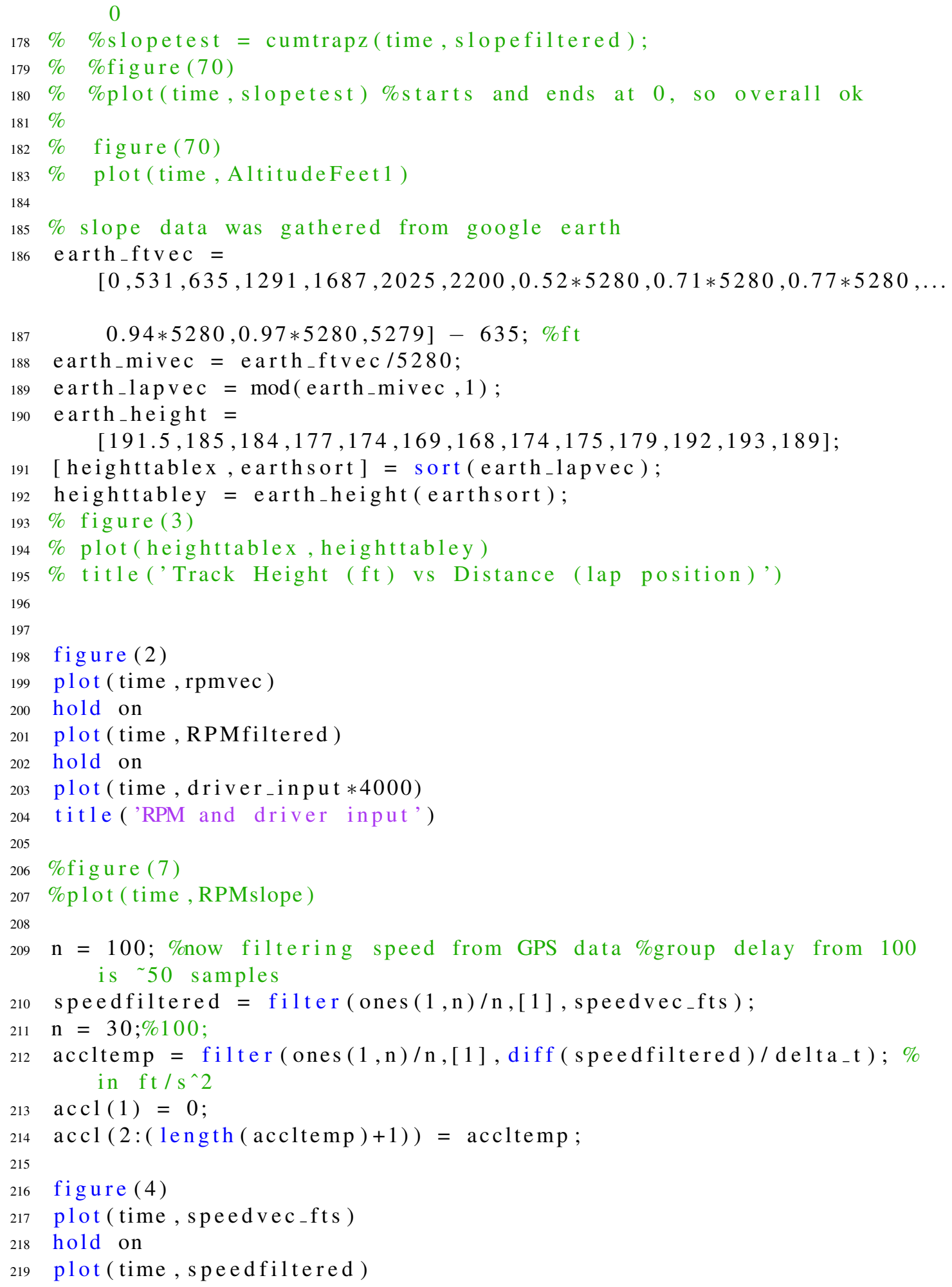




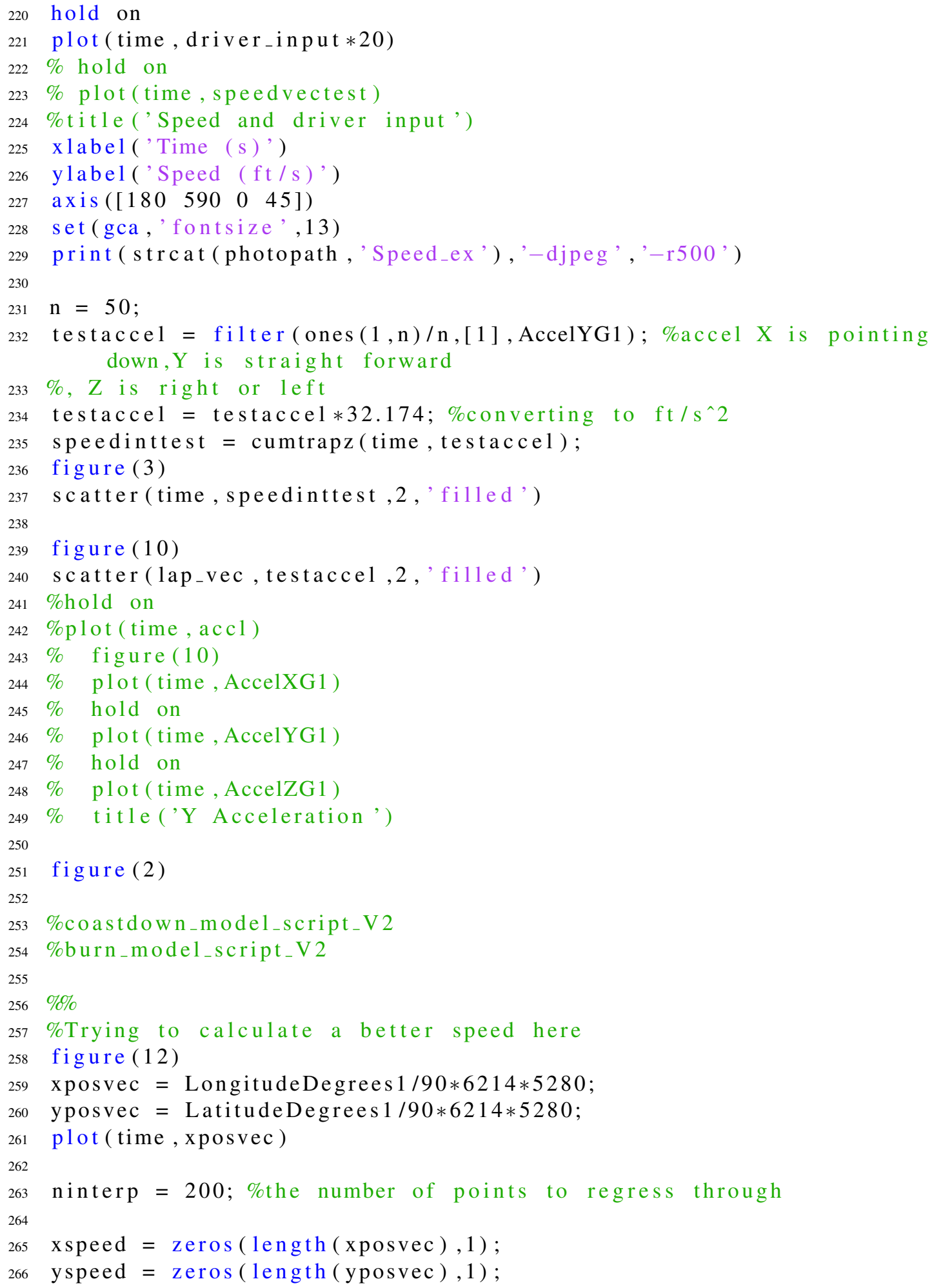




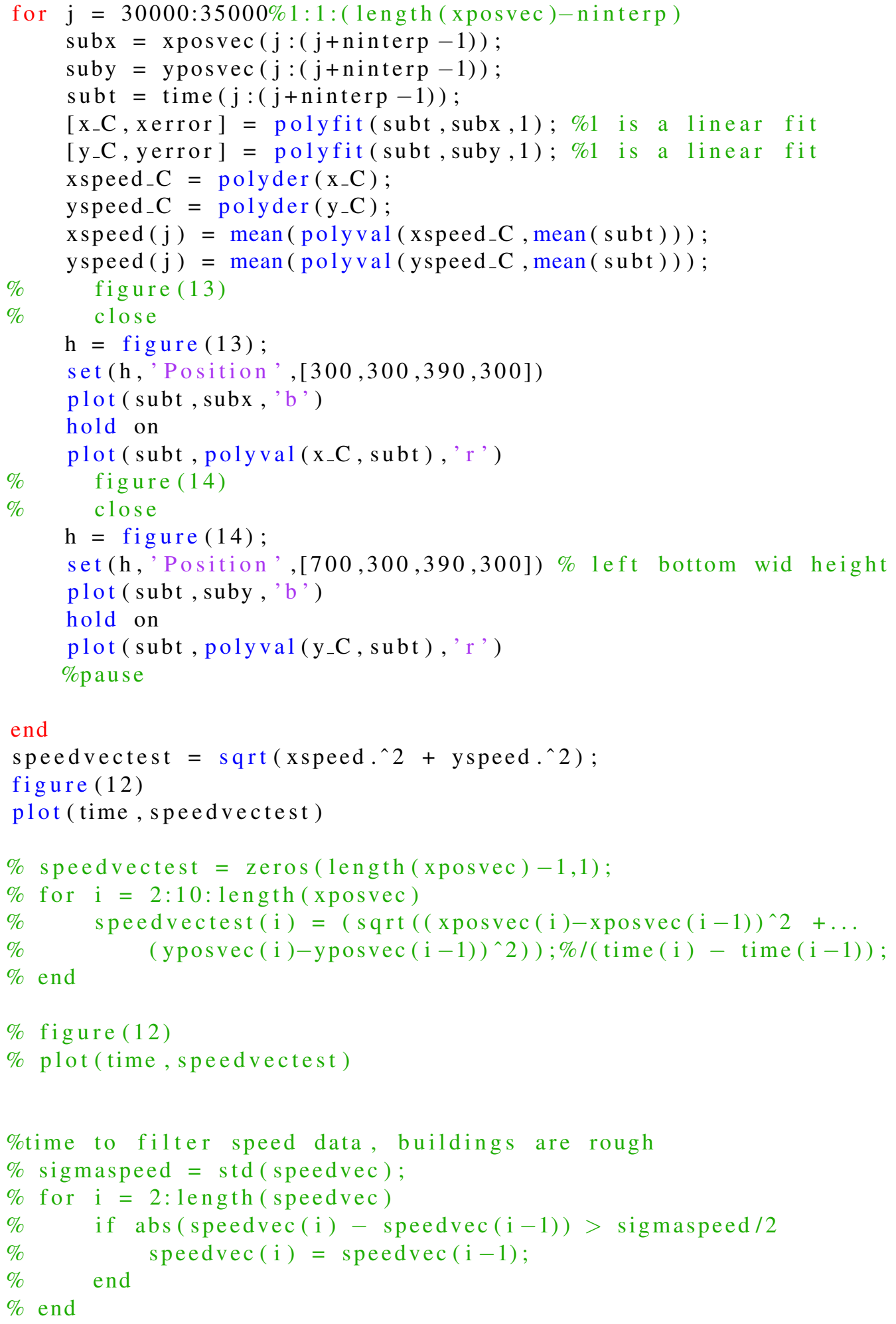




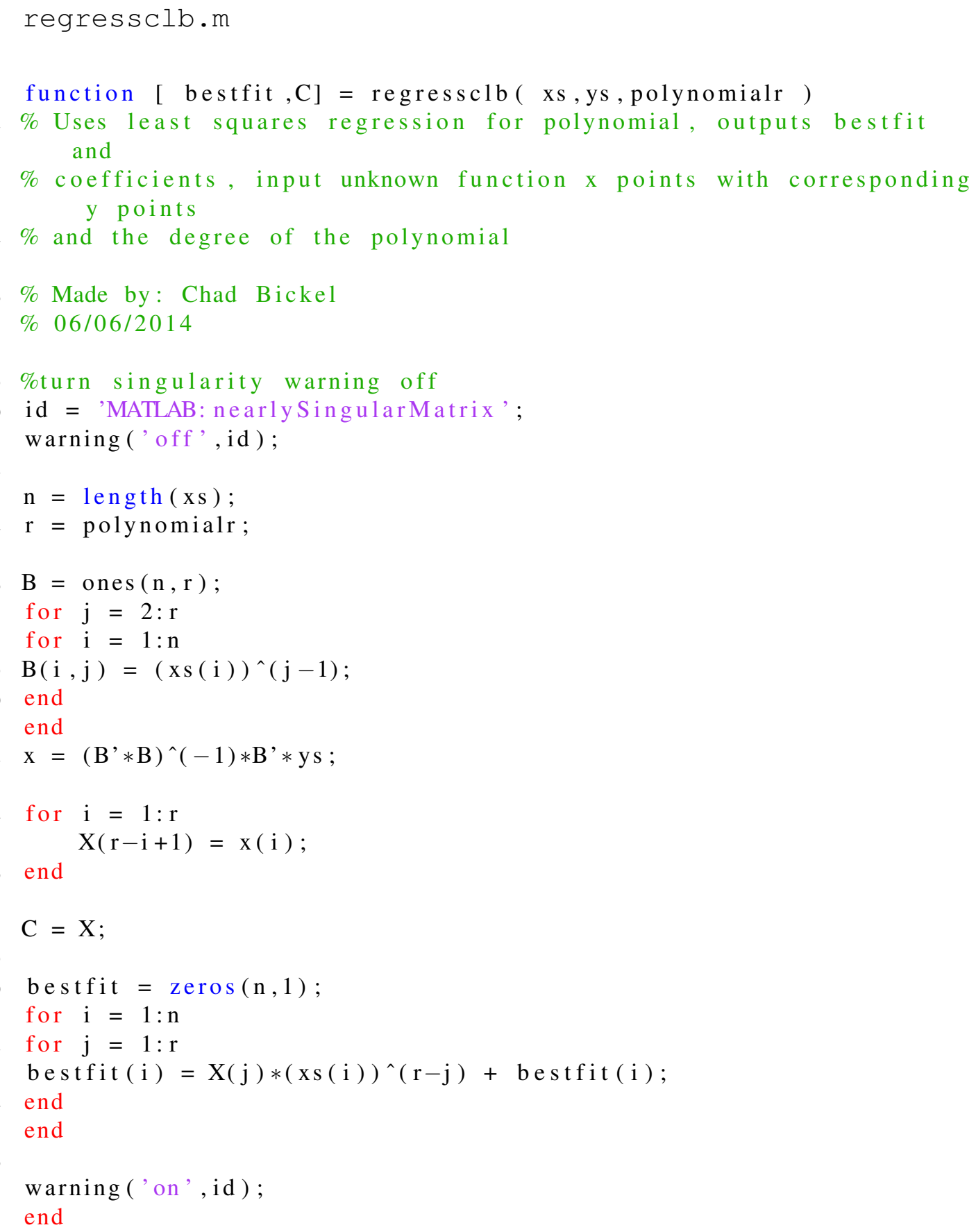




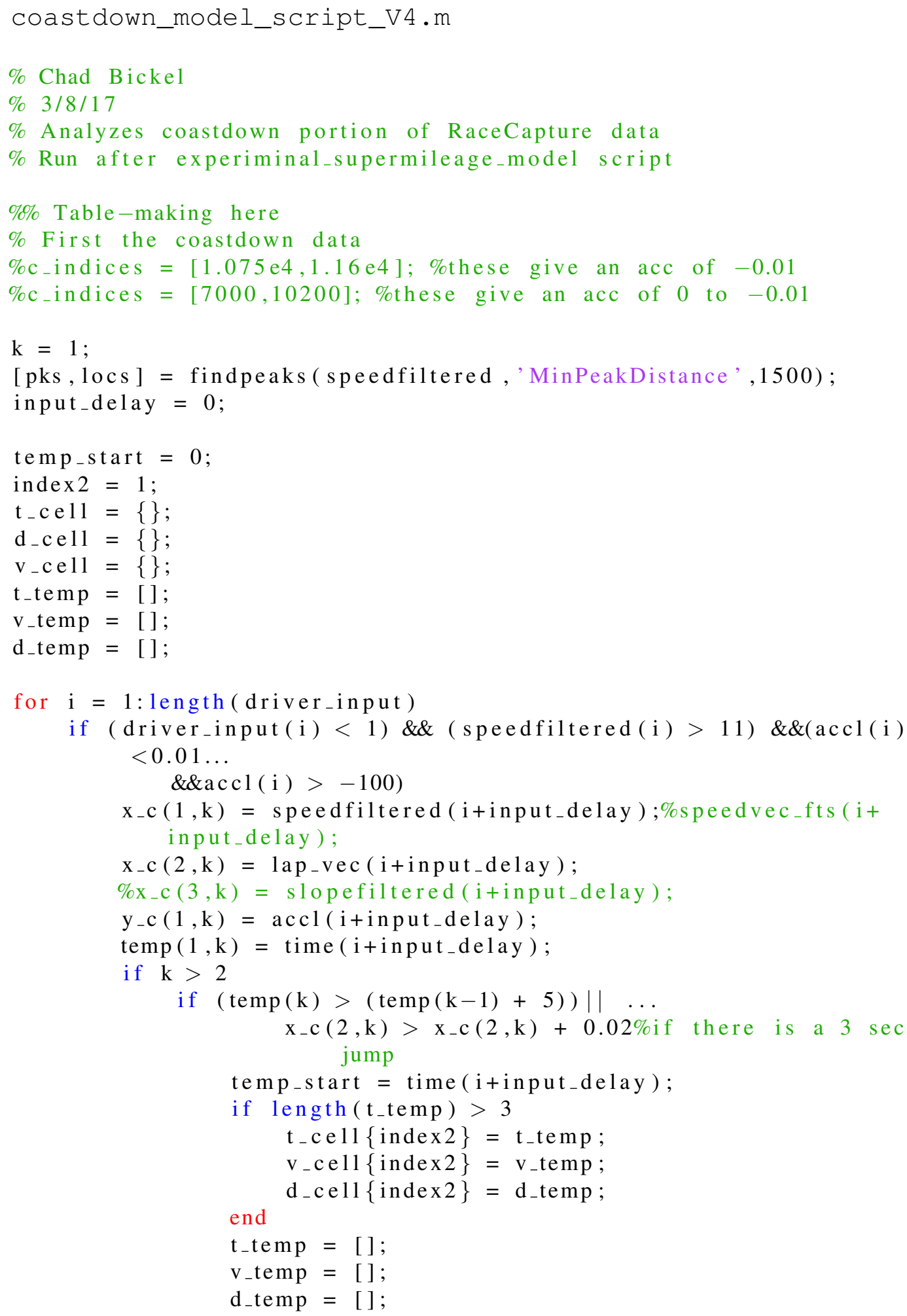




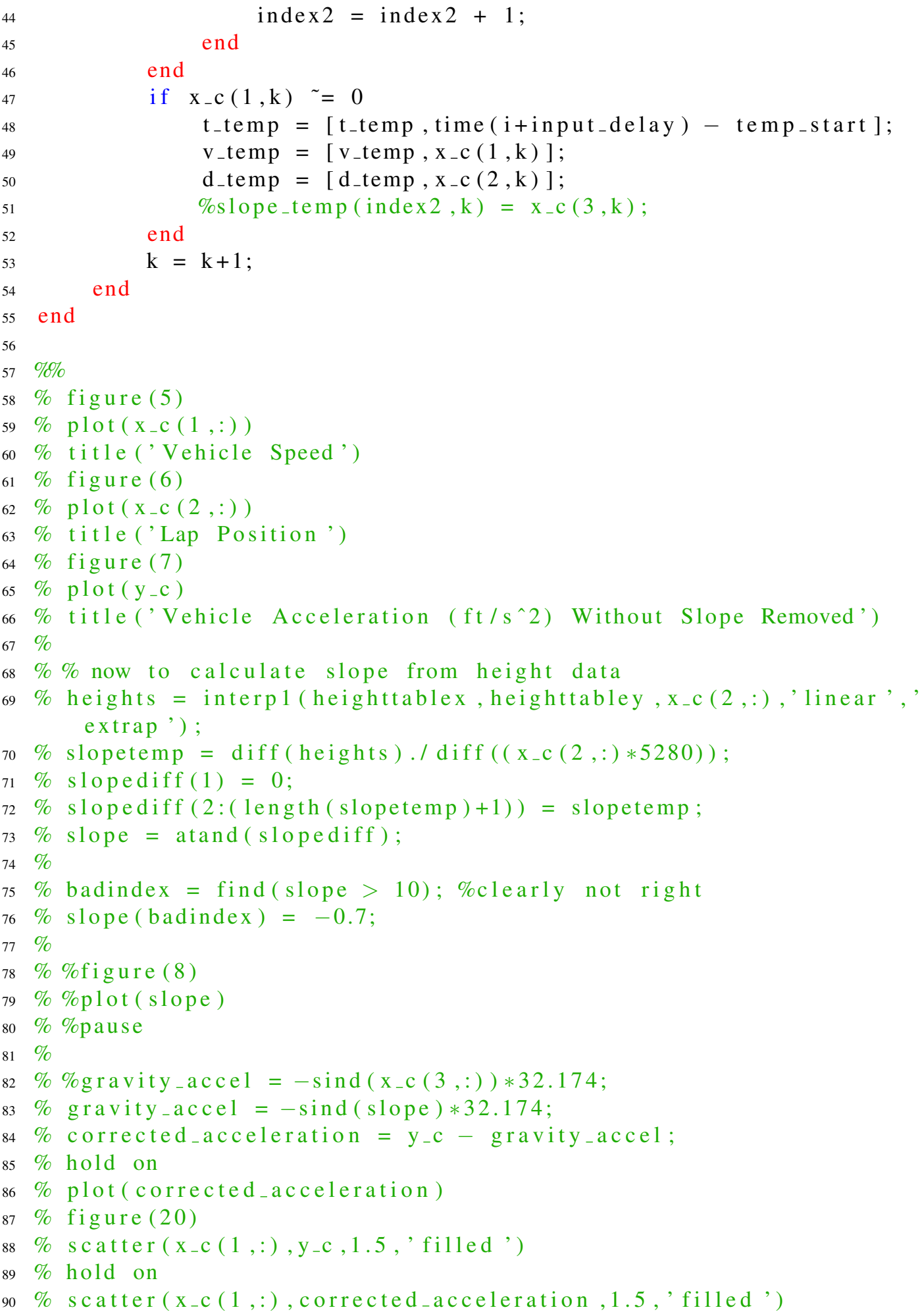




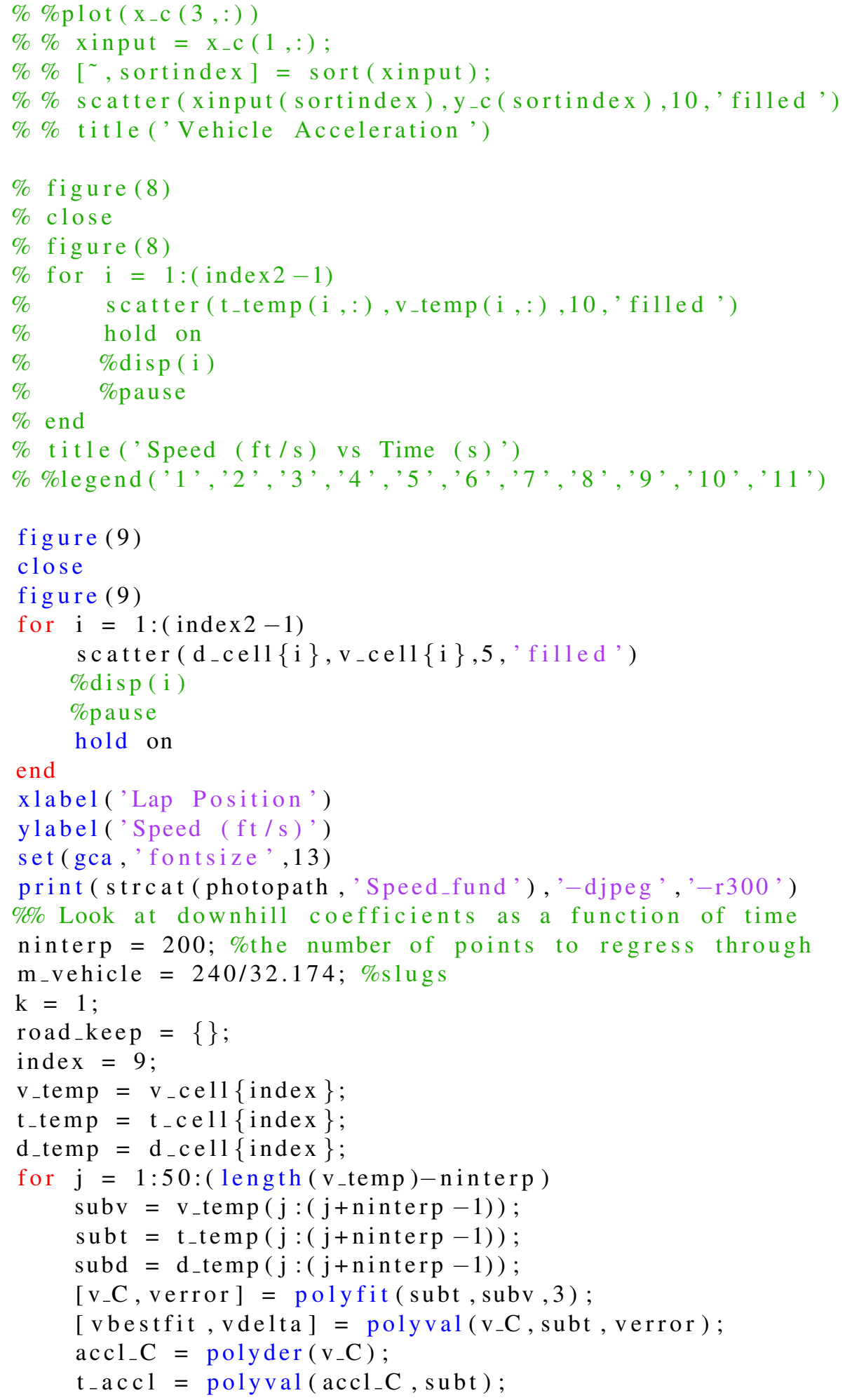


[road_C, aerror] = polyfit (vbestfit, t_accl*m_vehicle, 1$) ; \%$ subv, $\mathrm{t}_{-}$accl $* \mathrm{~m}_{-}$vehicle, 2$)$; 
211

212

213

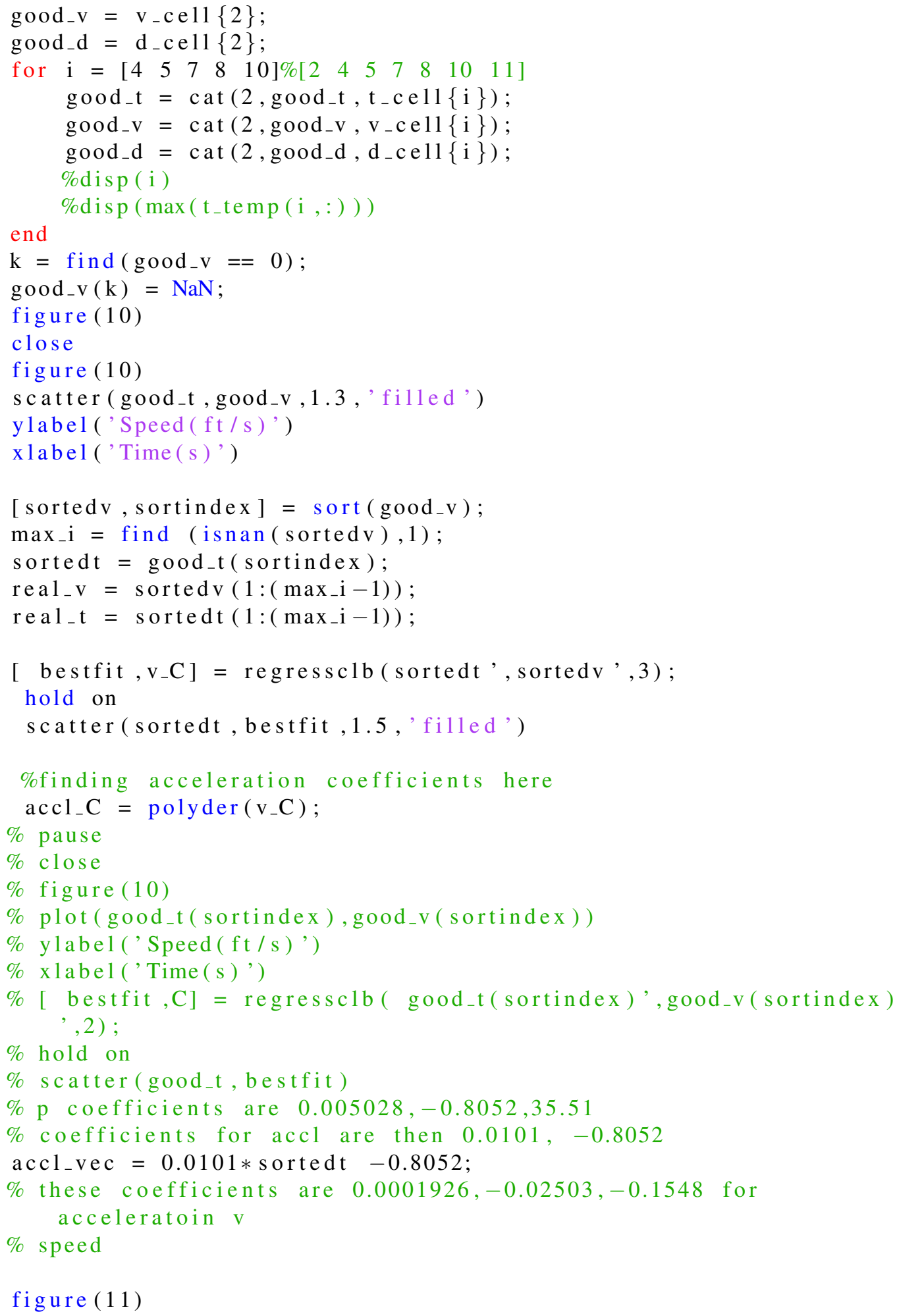




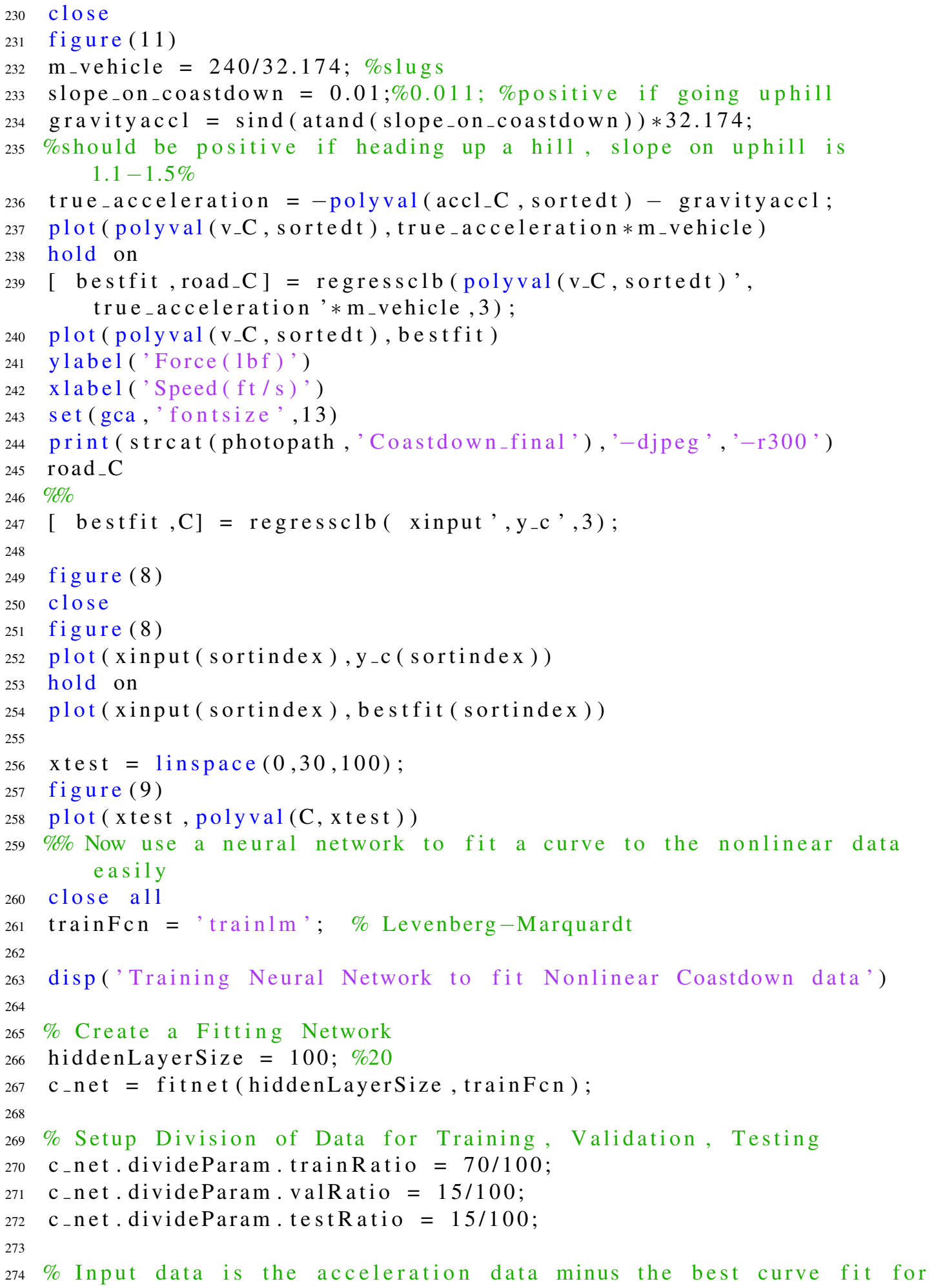




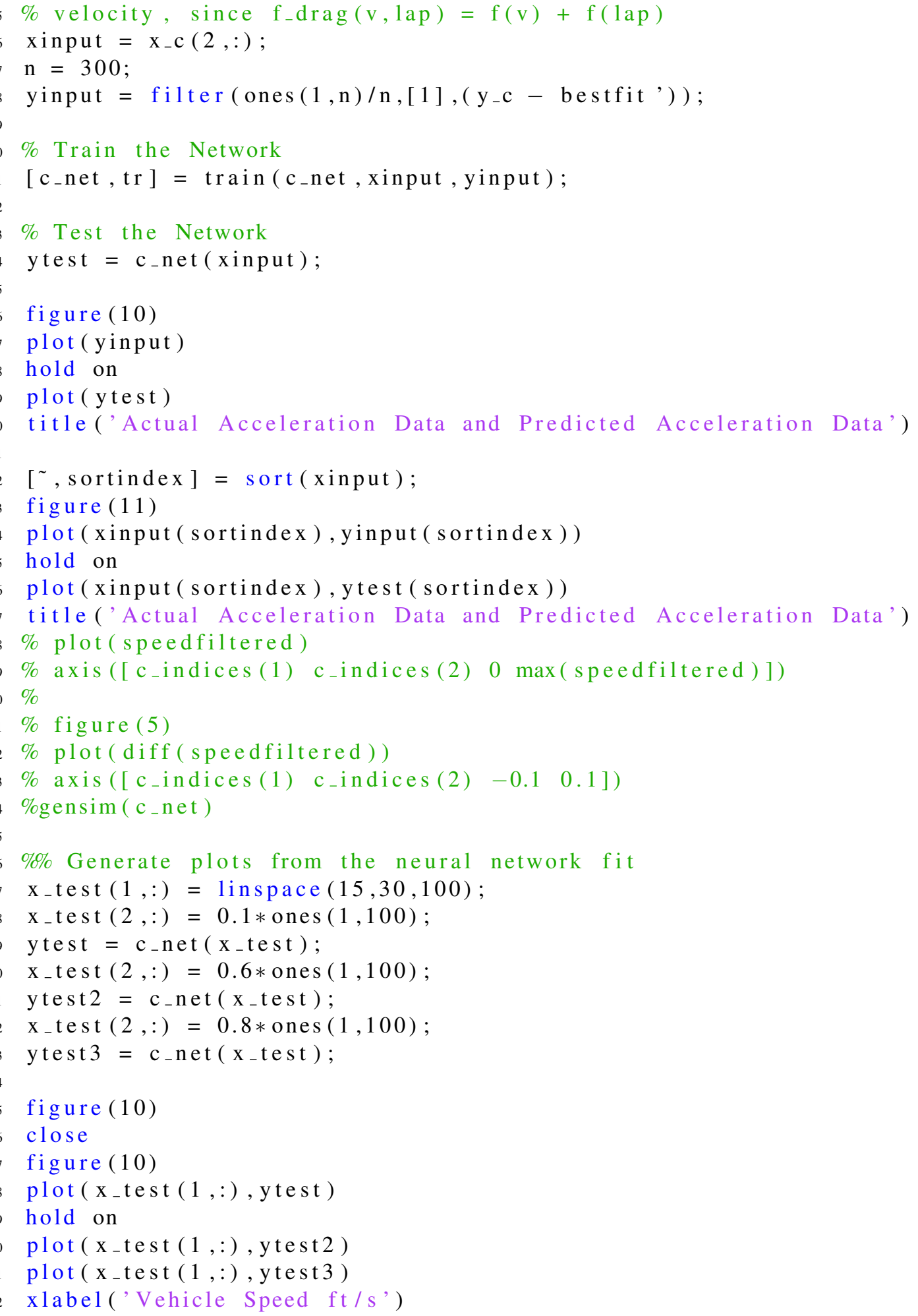




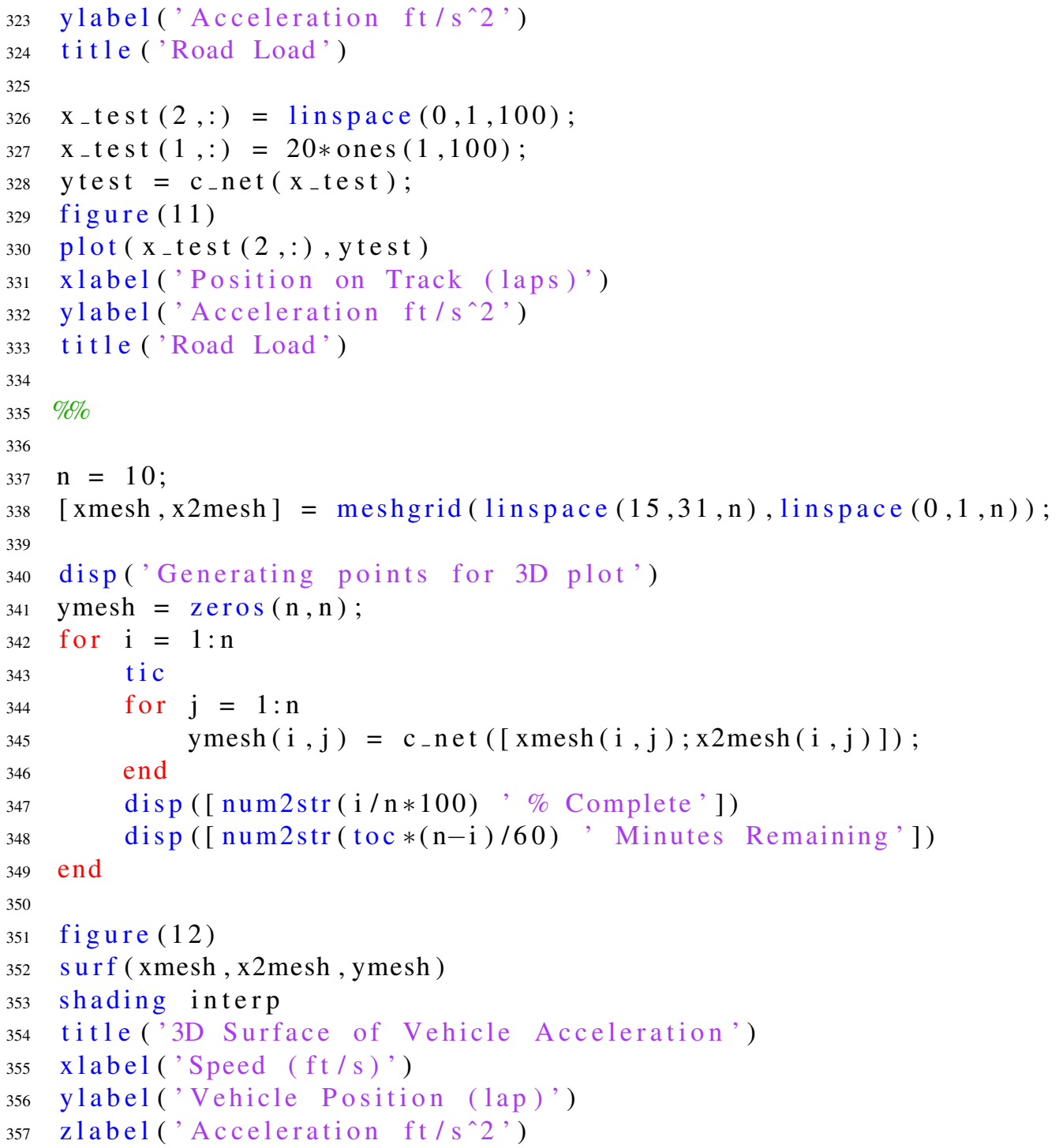




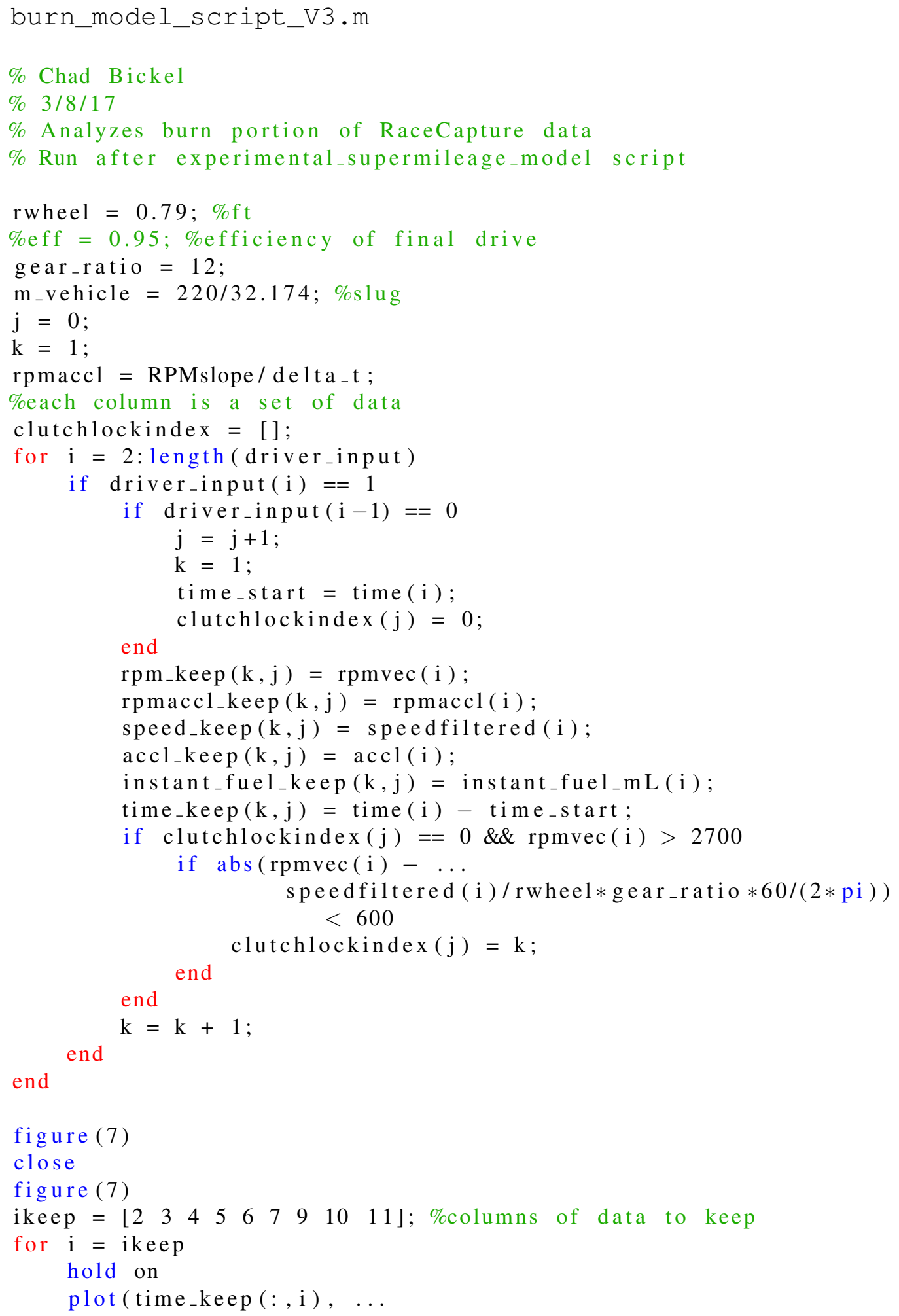


70

71

72

73

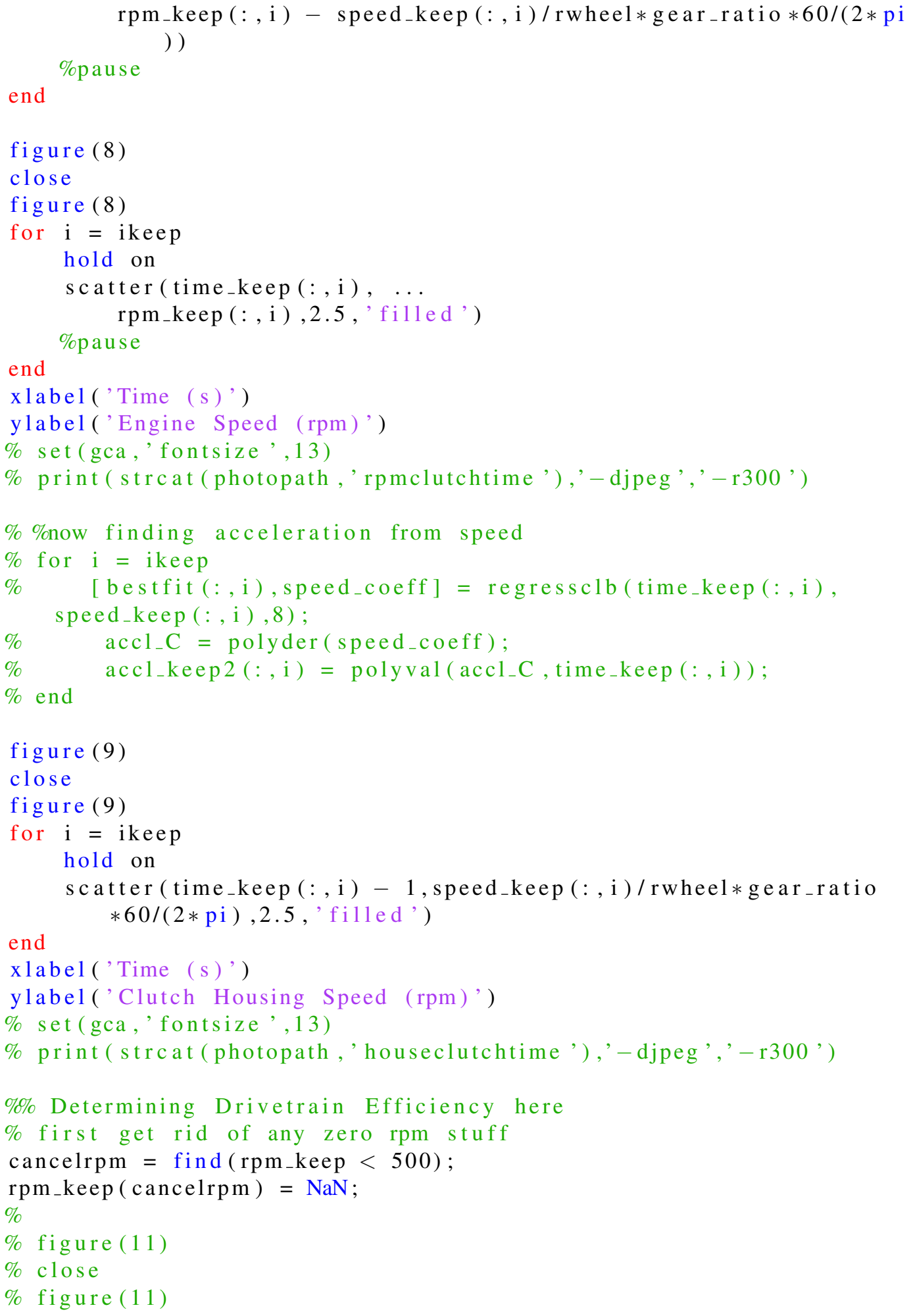




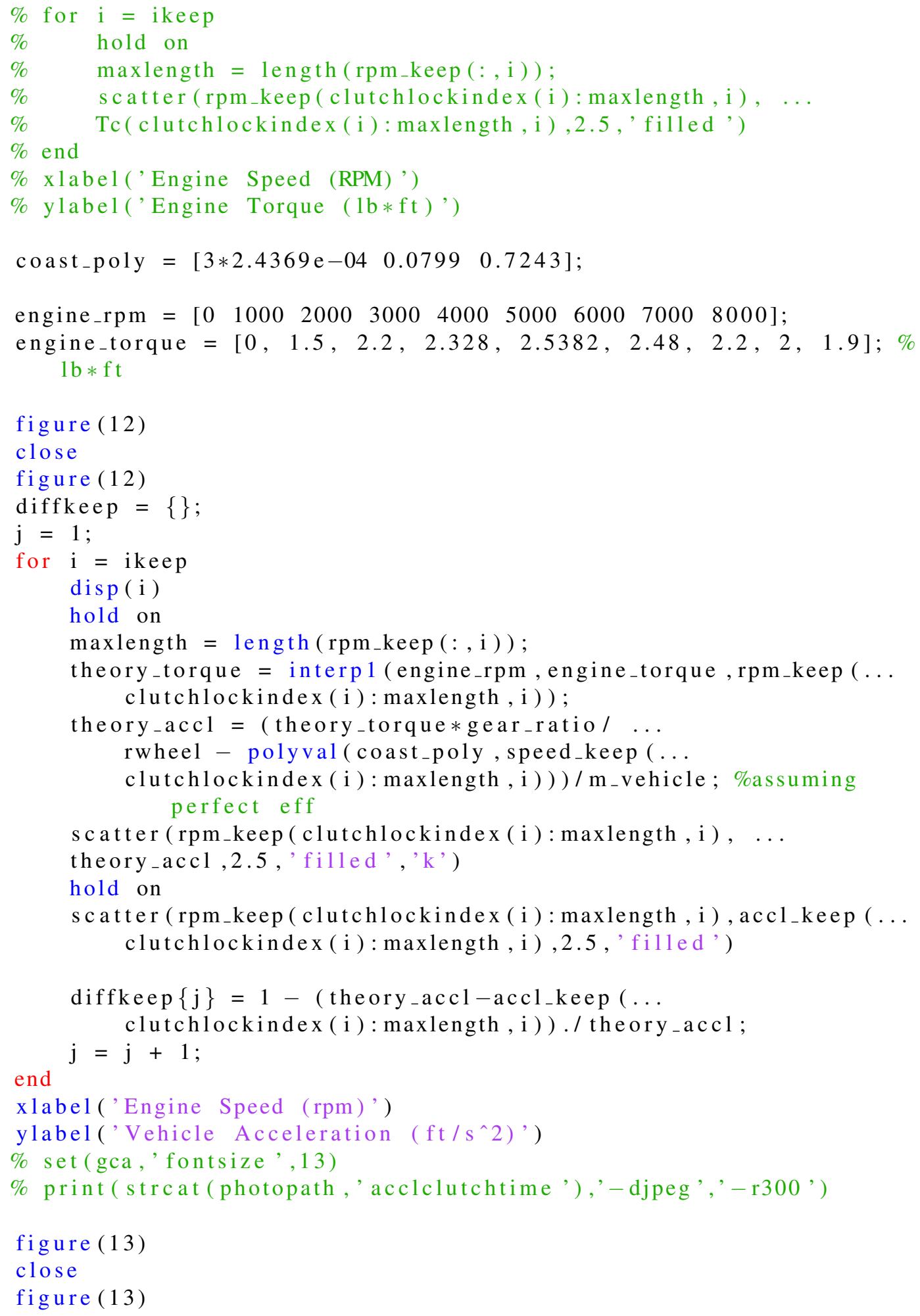




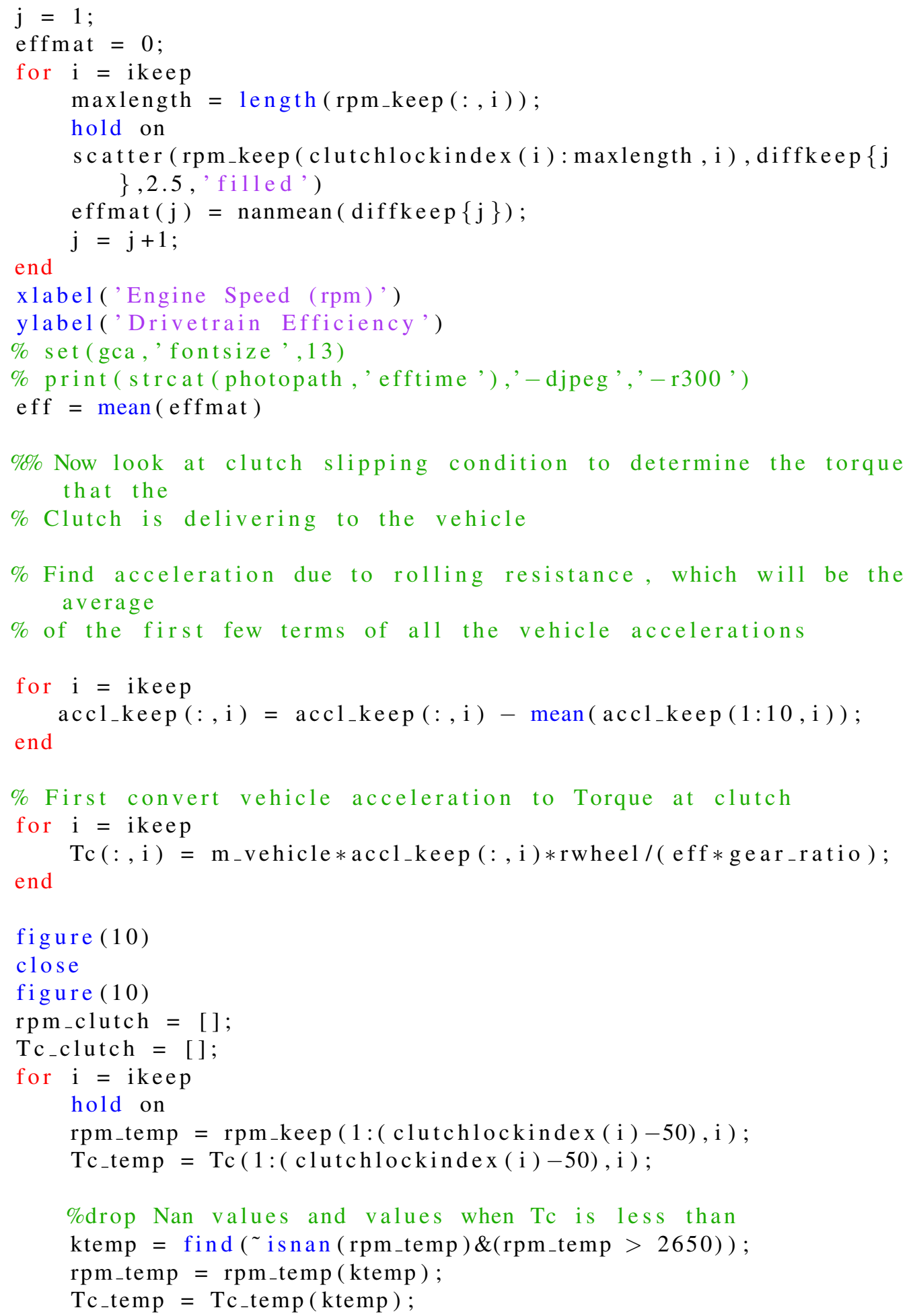




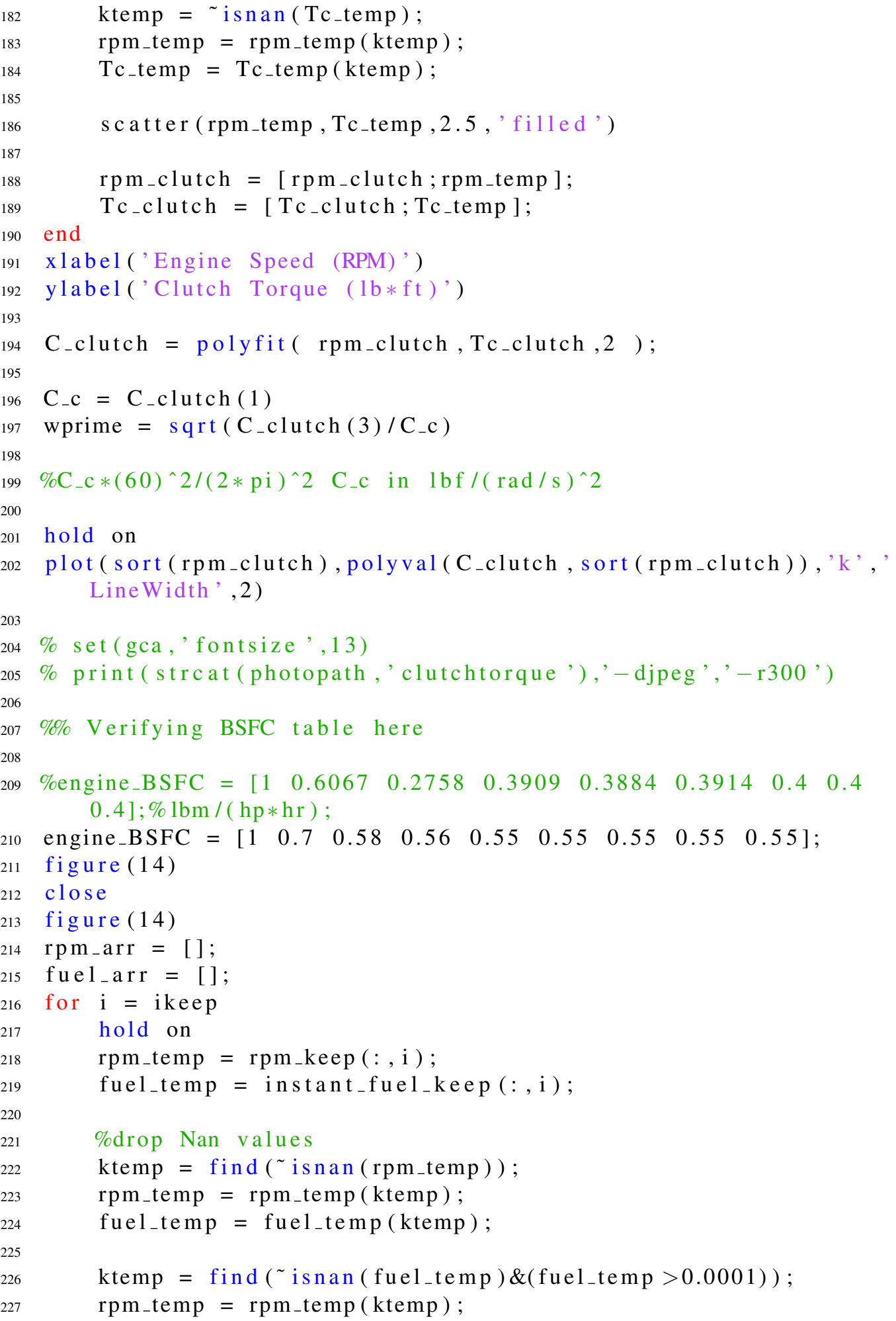




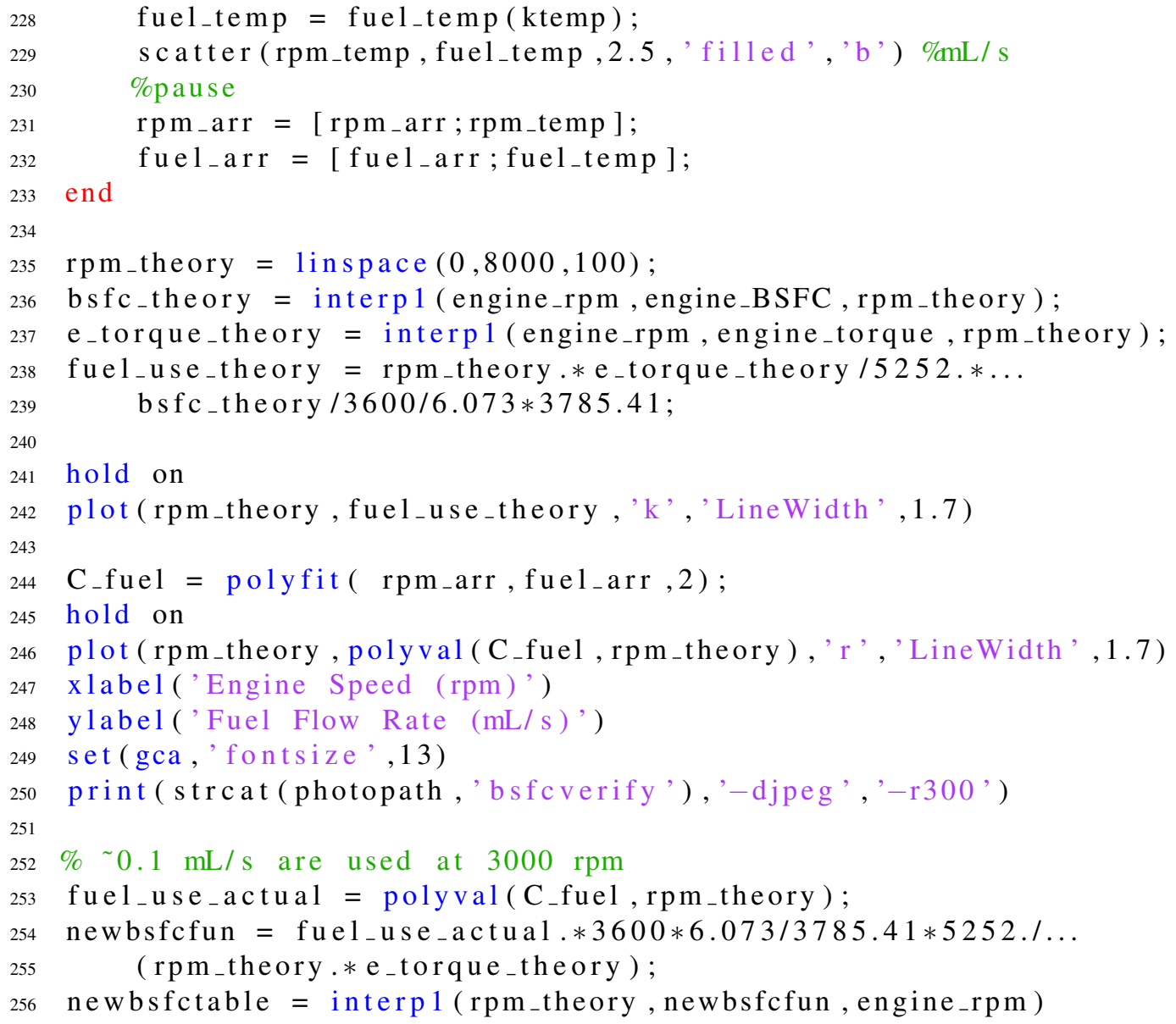




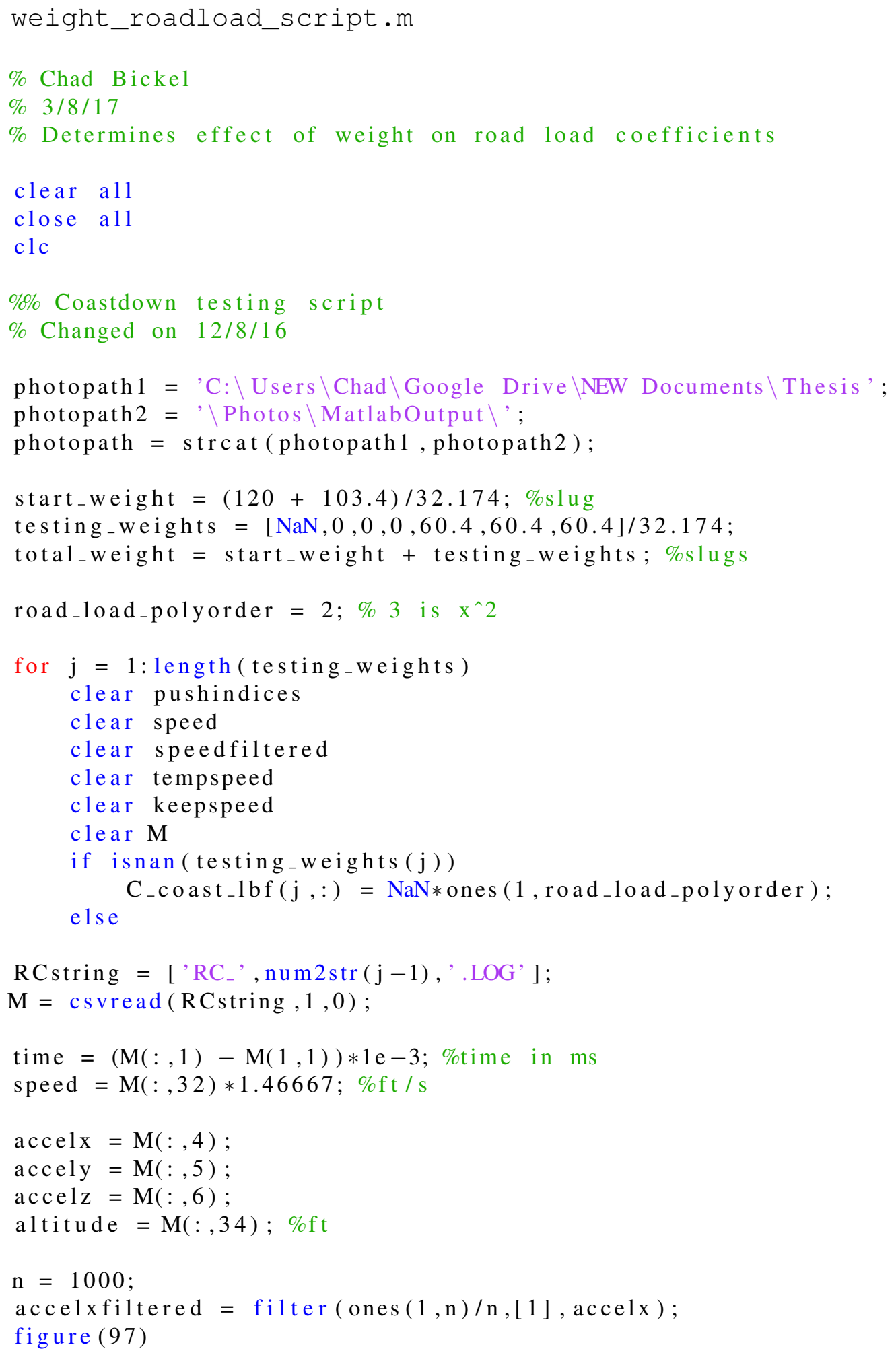




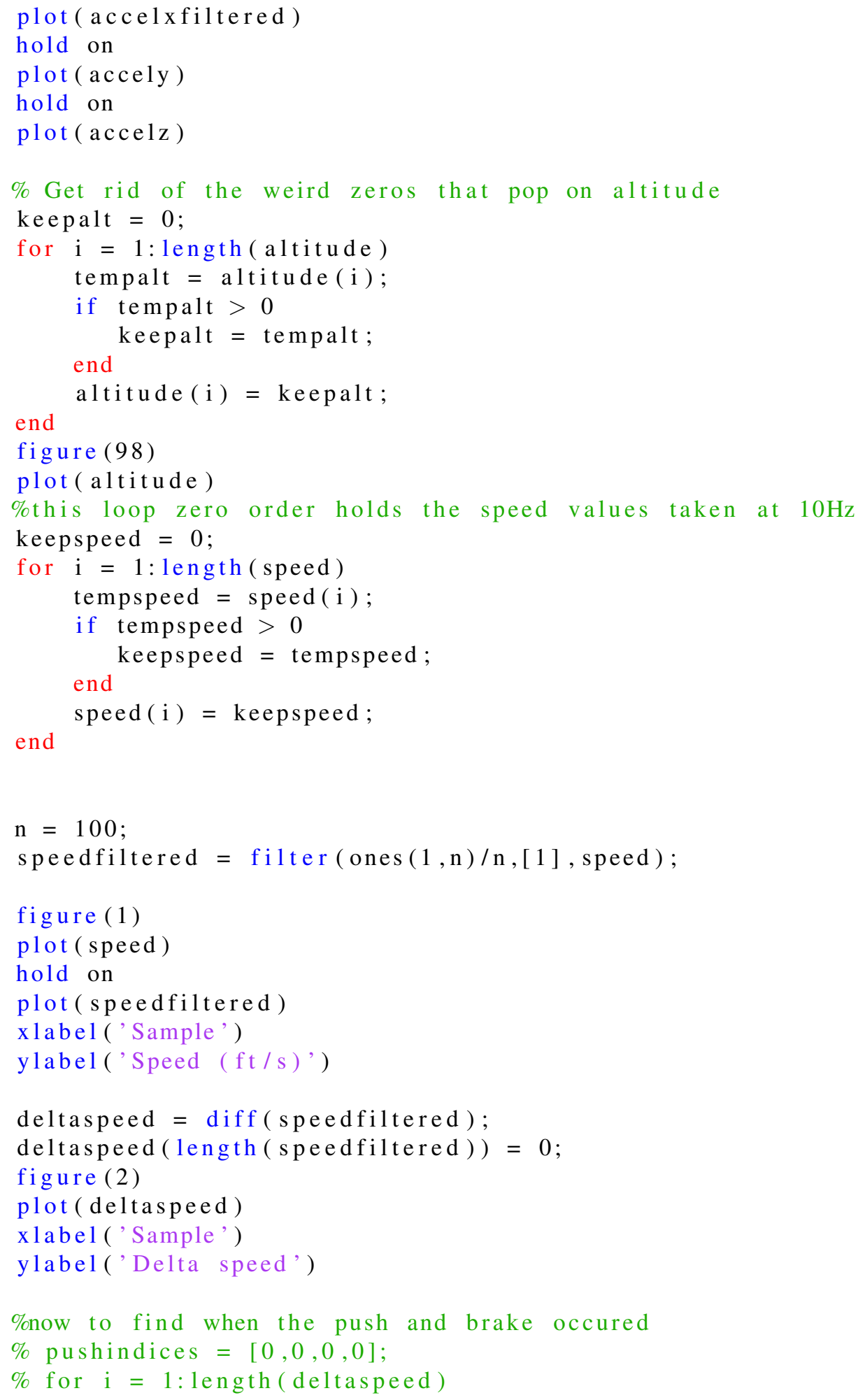




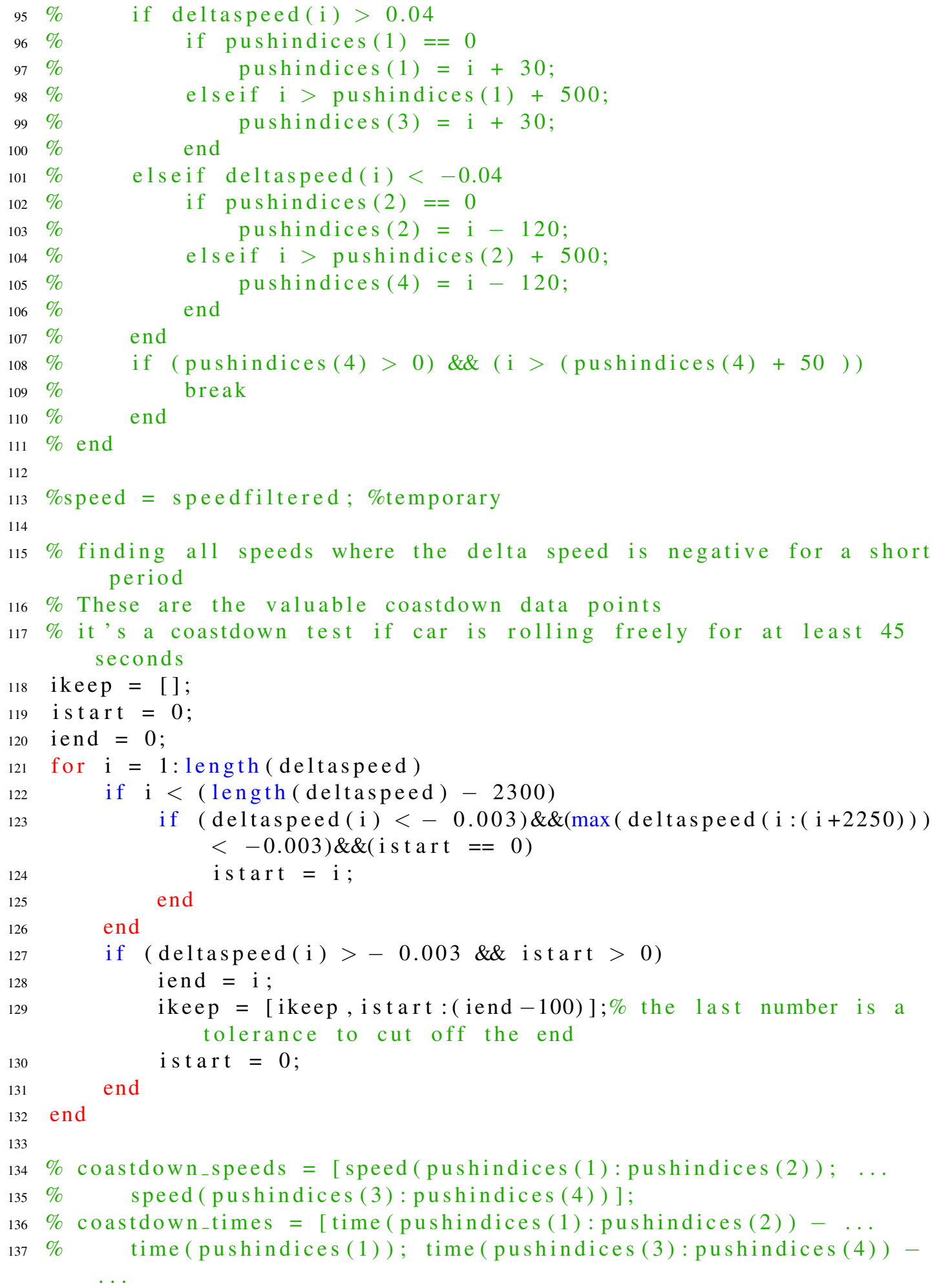




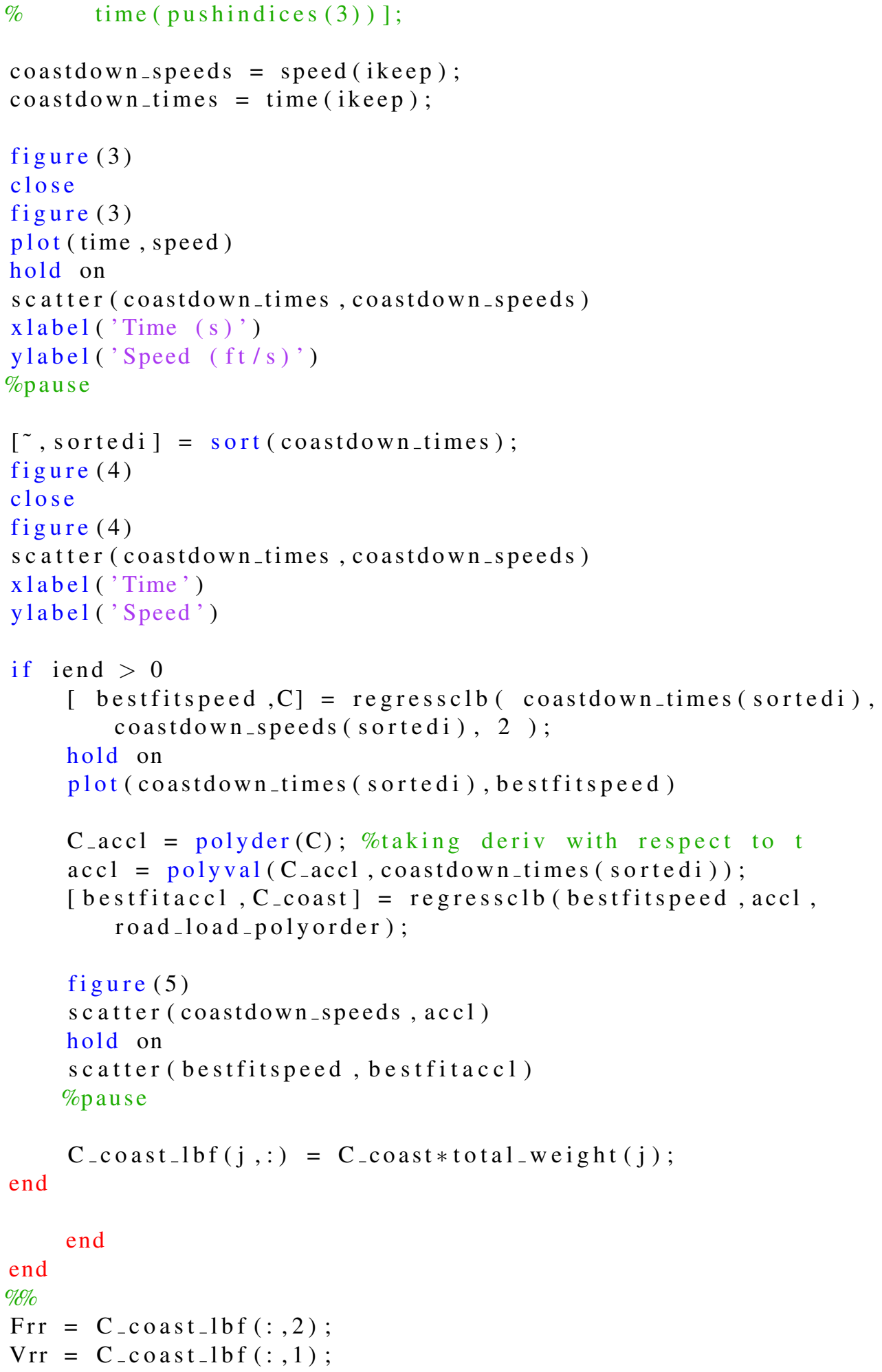




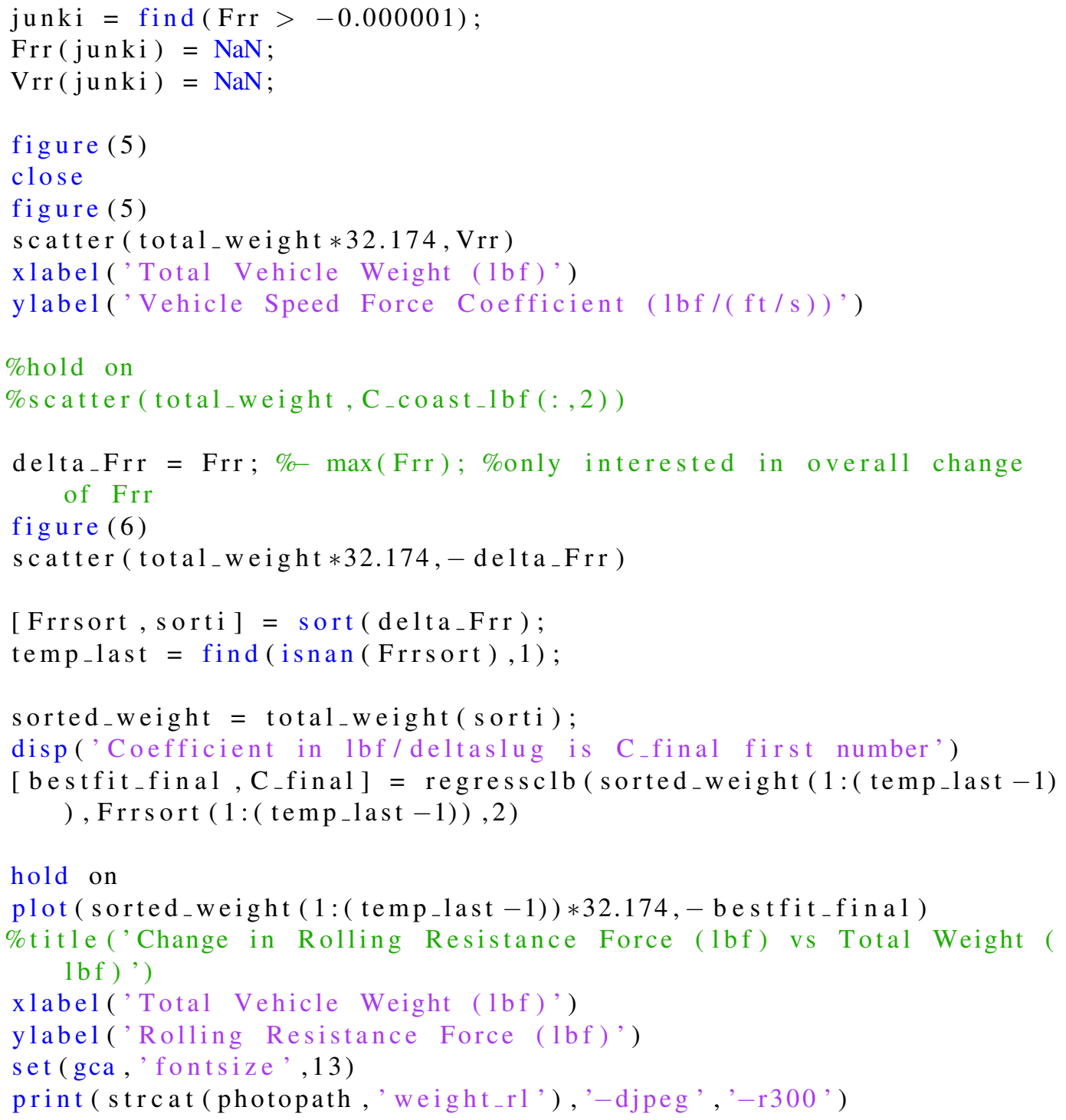




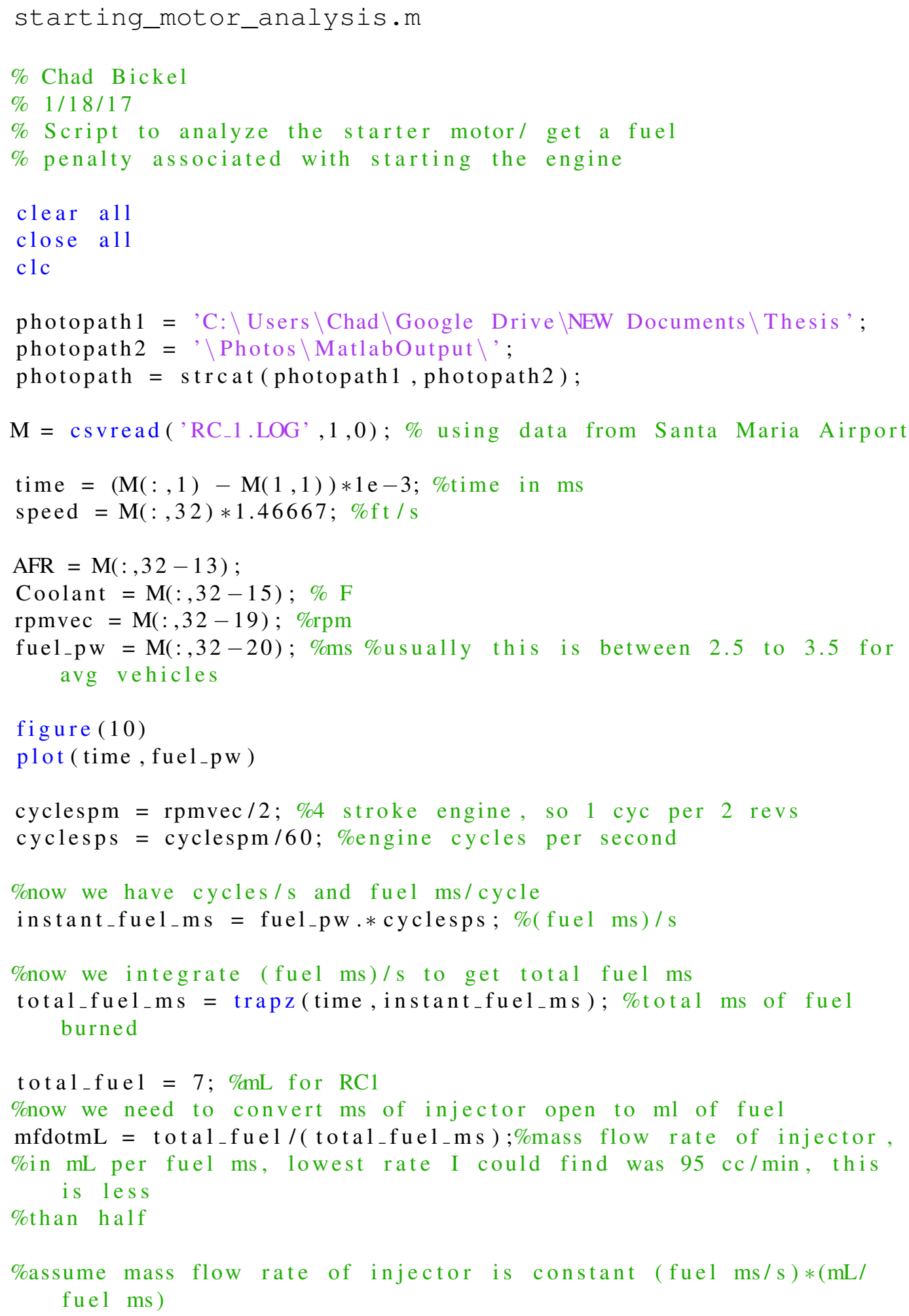




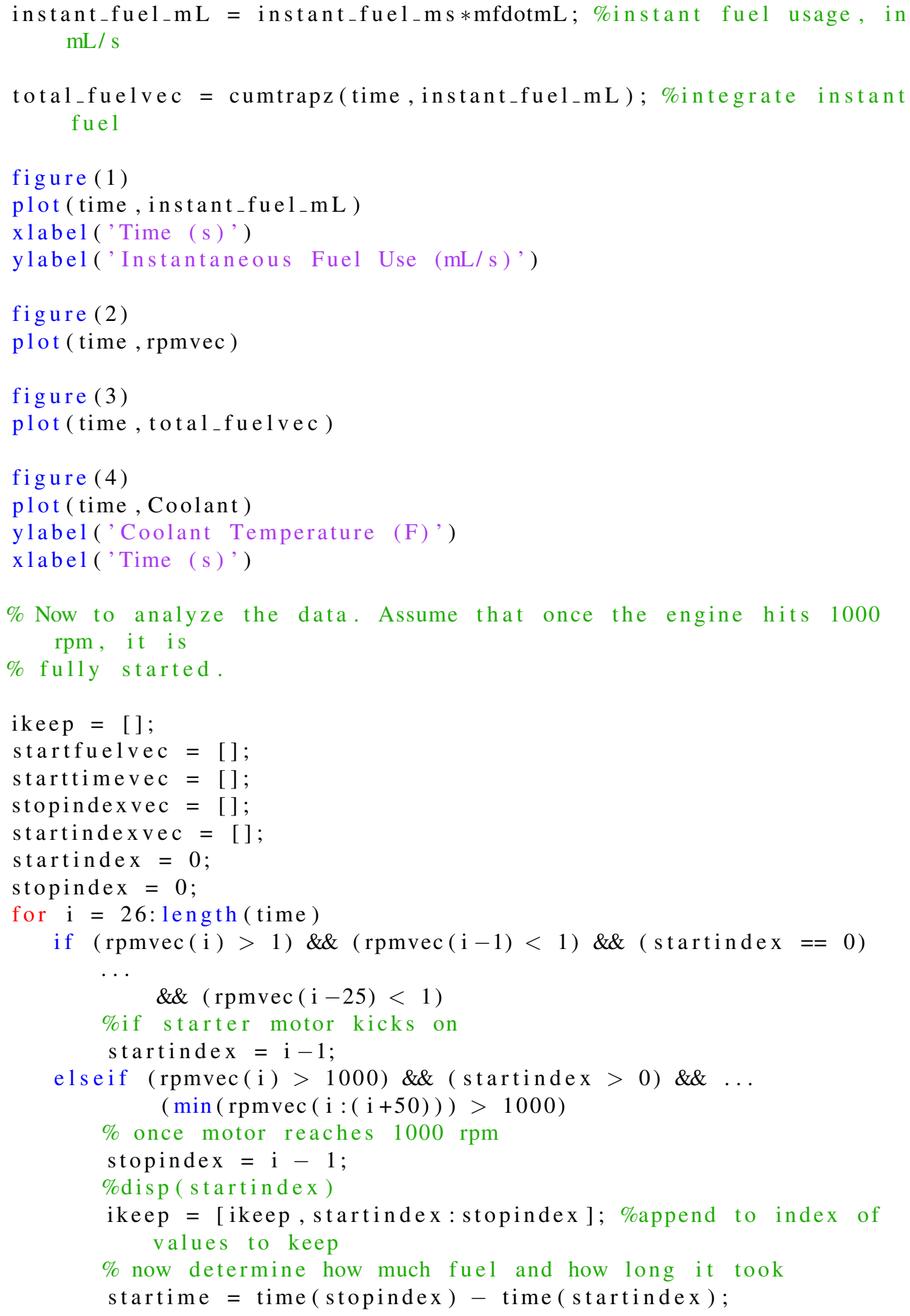


125

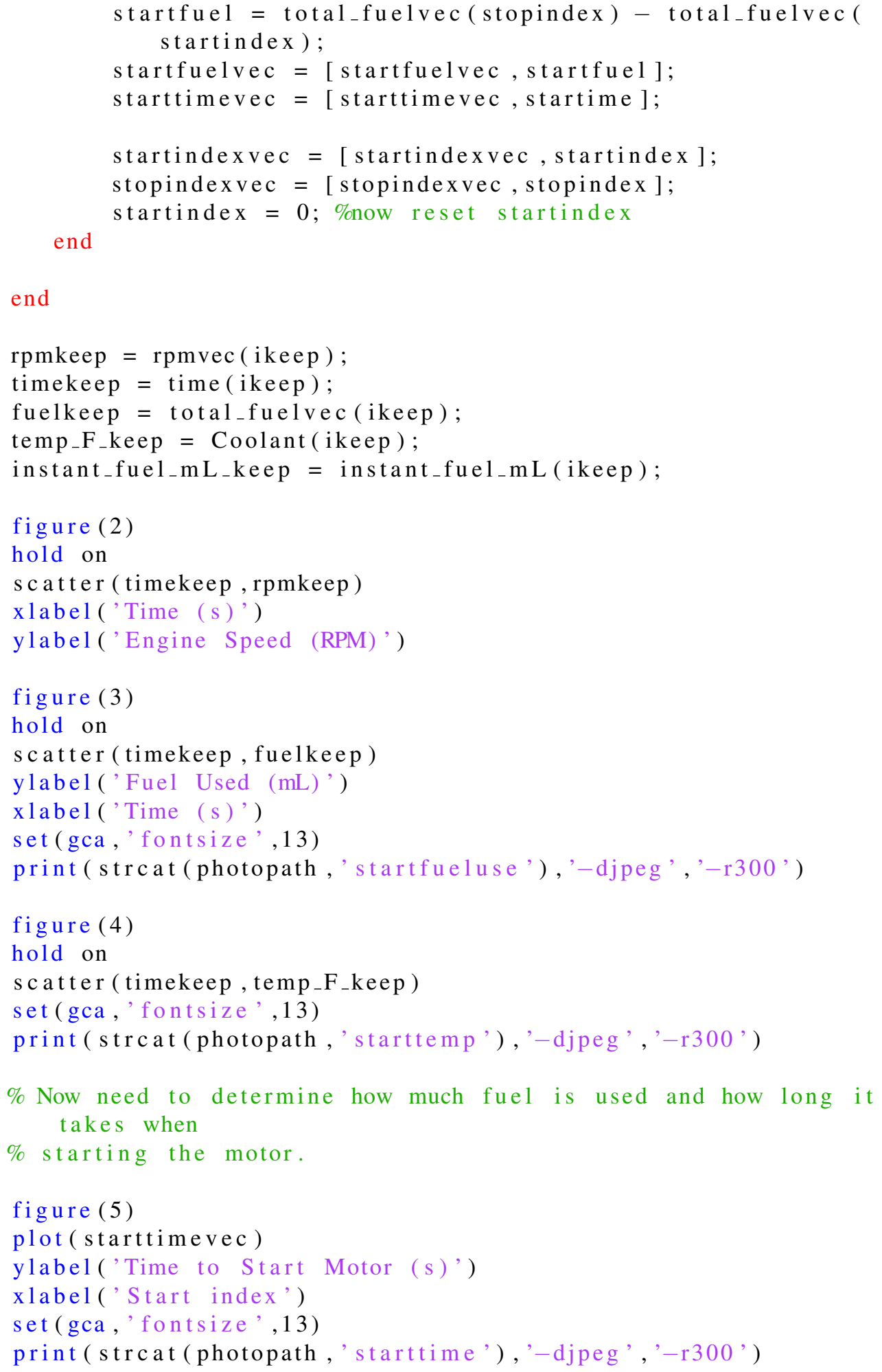


146

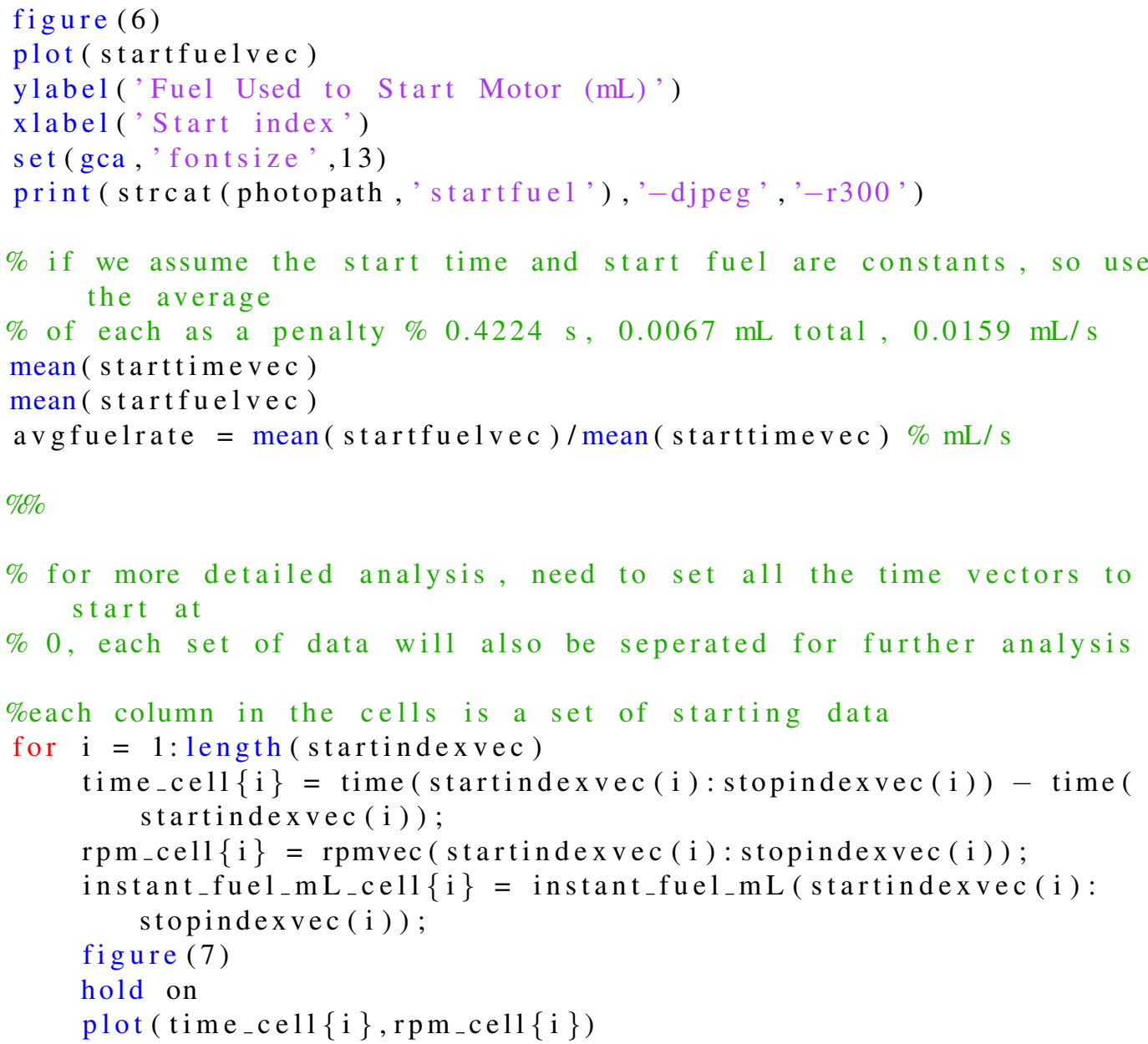




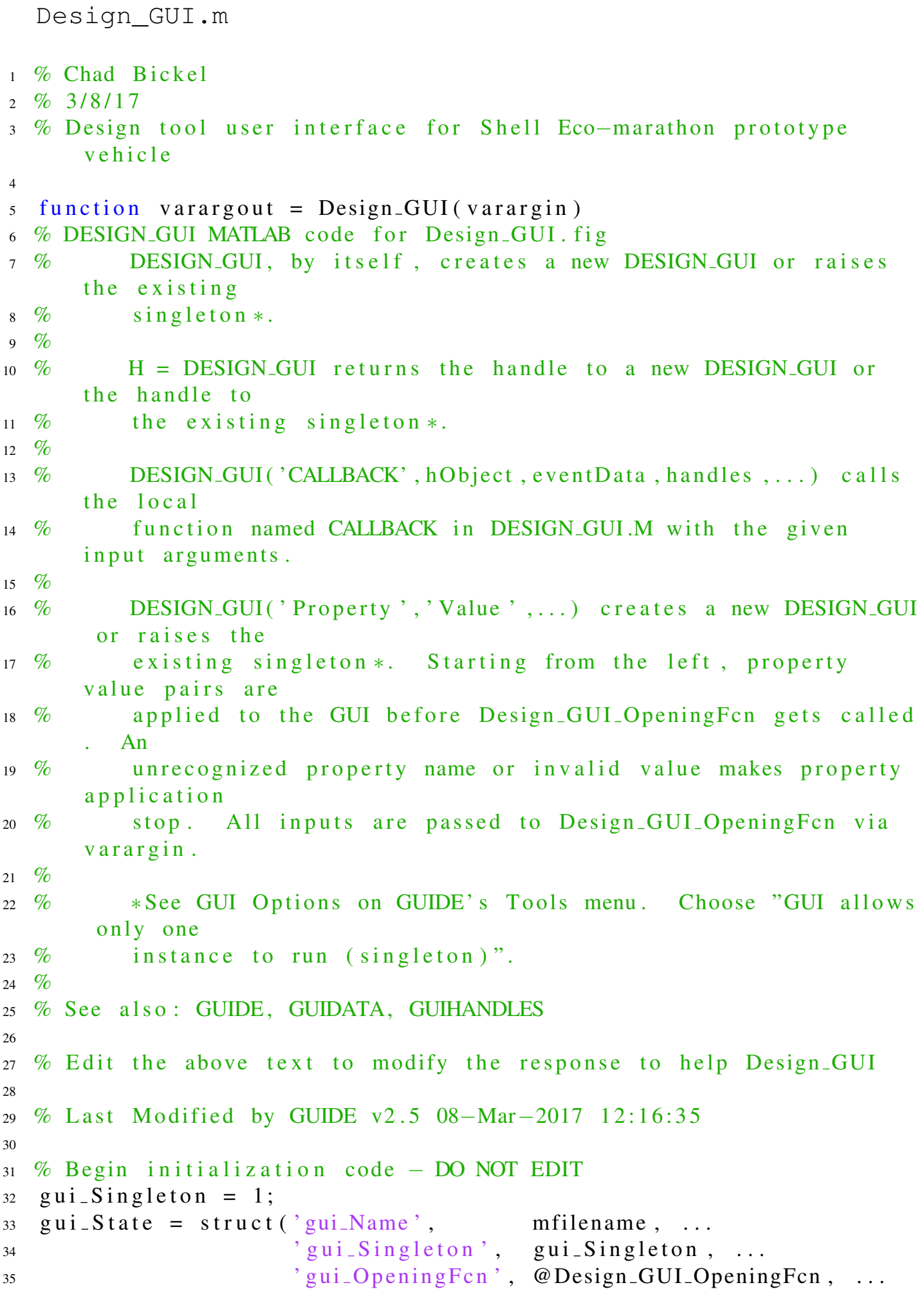




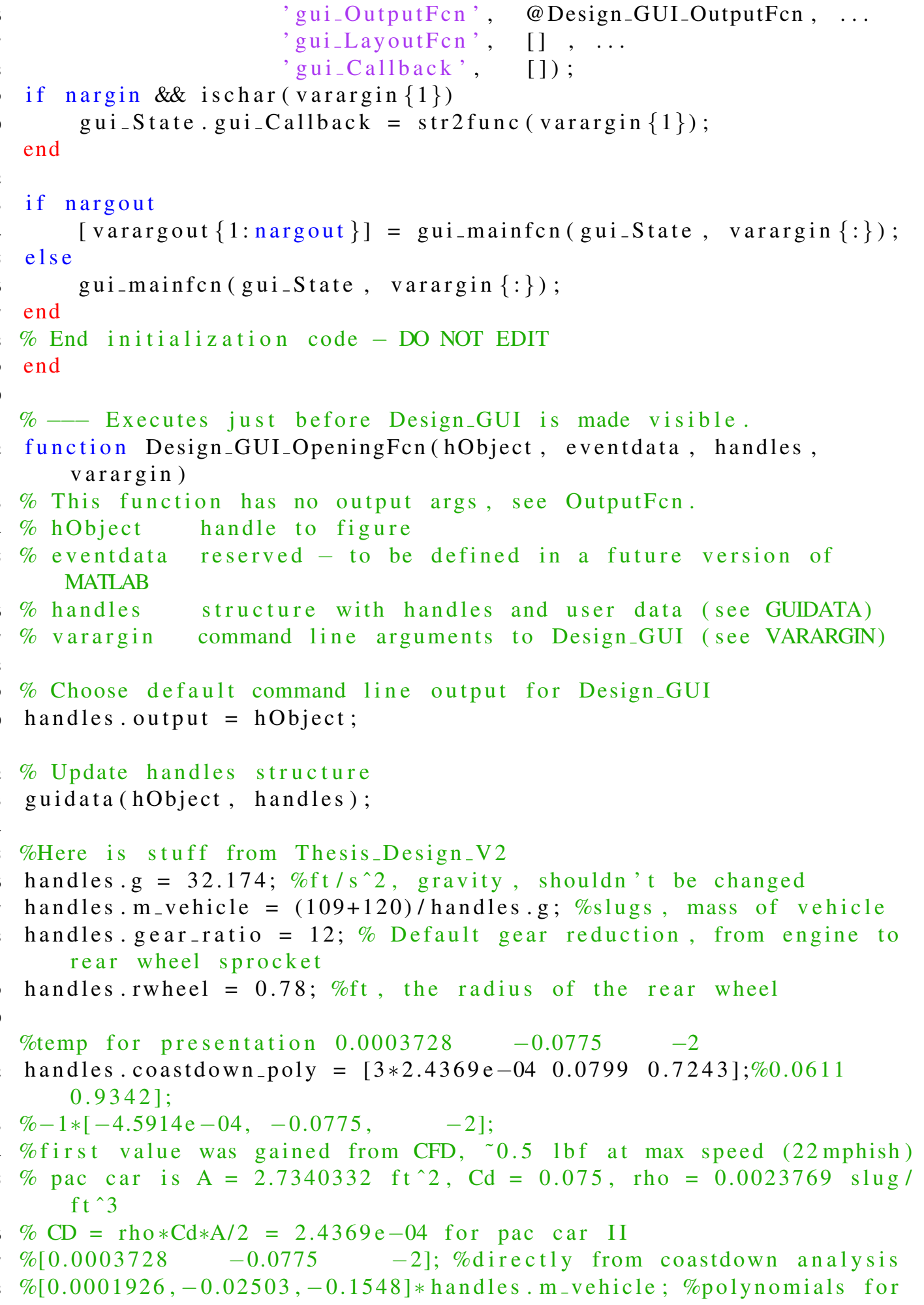




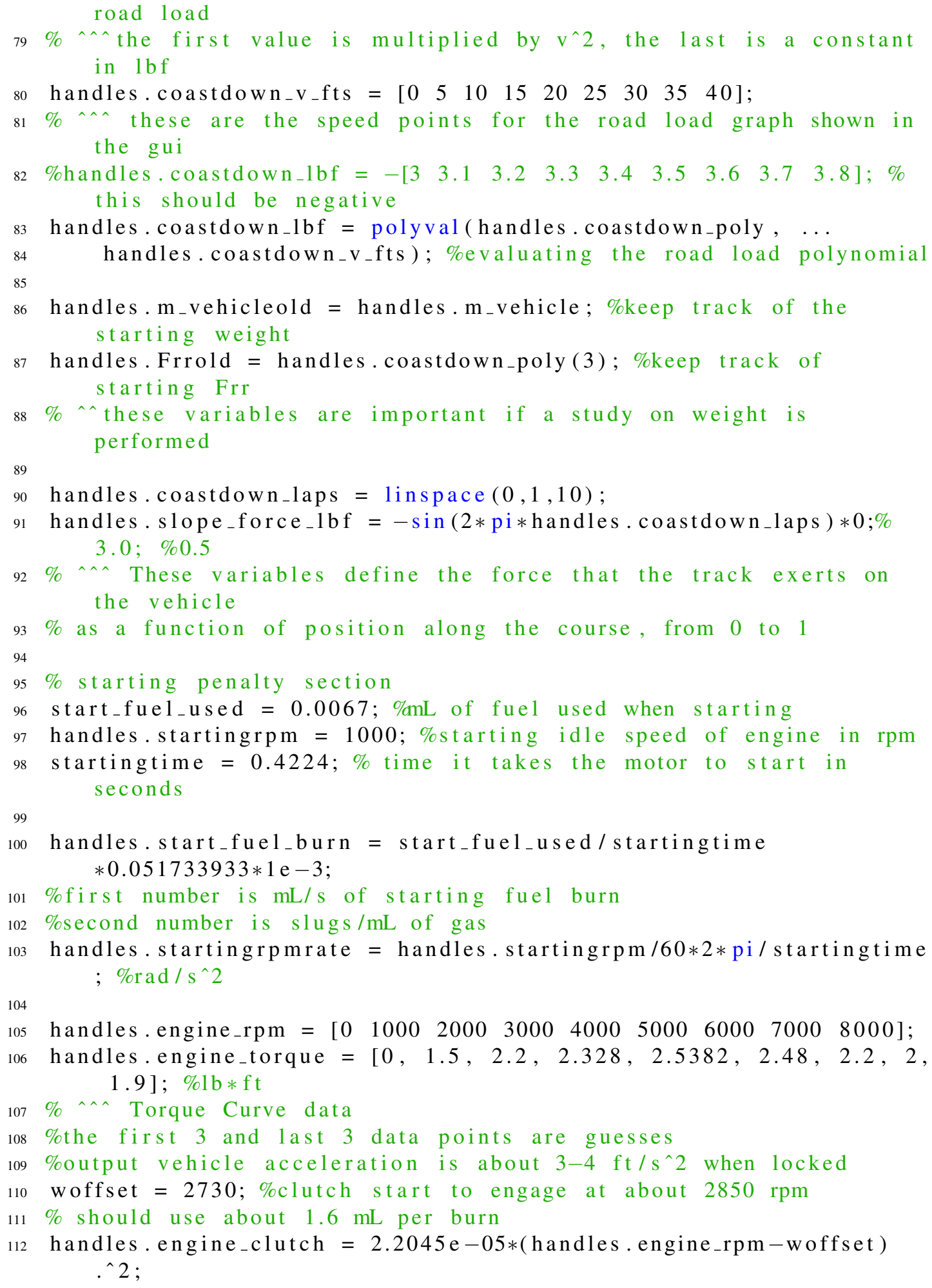

handles.start_fuel_burn $=$ start_fuel_used/startingtime $* 0.051733933 * 1 \mathrm{e}-3$;

\%first number is $\mathrm{mL} / \mathrm{s}$ of starting fuel burn

\%second number is slugs/mL of gas

handles . startingrpmrate $=$ handles startingrpm $/ 60 * 2 *$ pi $/$ startingtime ; \% rad / s^2

handles.engine_rpm $=\left[\begin{array}{llllllllll}0 & 1000 & 2000 & 3000 & 4000 & 5000 & 6000 & 7000 & 8000\end{array}\right] ;$

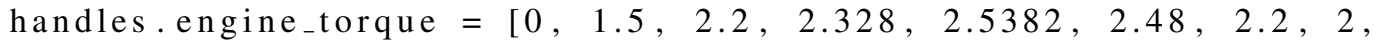
$1.9] ; \% 1 \mathrm{~b} * \mathrm{ft}$

$\% \wedge$ Torque Curve data

\%the first 3 and last 3 data points are guesses

\%output vehicle acceleration is about 3-4 ft/s^2 when locked

woffset $=2730 ; \%$ clutch start to engage at about $2850 \mathrm{rpm}$

$\%$ should use about $1.6 \mathrm{~mL}$ per burn

handles.engine $\_$clutch $=2.2045 \mathrm{e}-05 *($ handles.engine_rpm-woffset $)$

$\wedge 2$; 


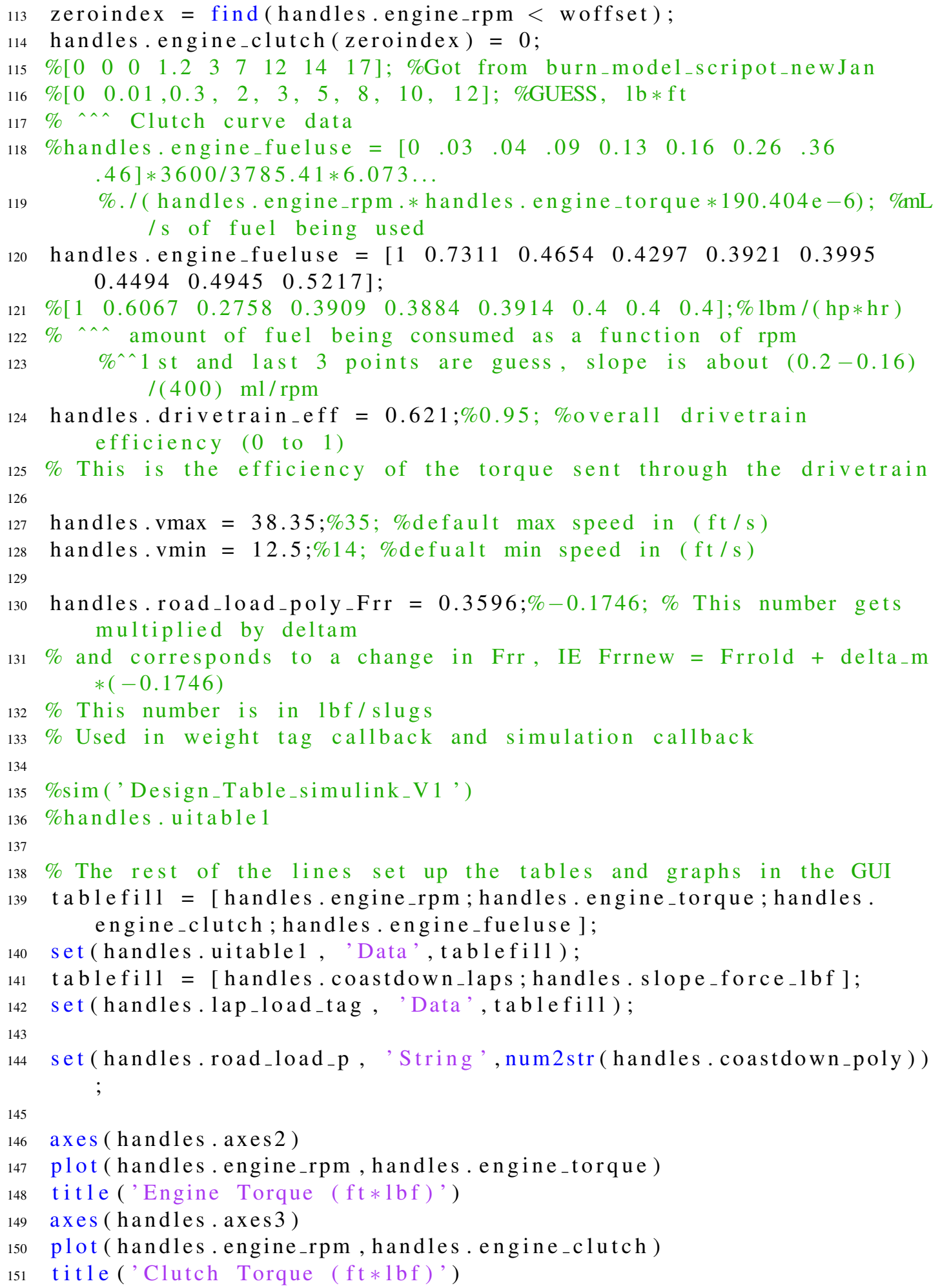


axes (handles.axes4)

plot (handles.engine_rpm, handles.engine_fueluse)

title ('BSFC( $1 \mathrm{~b} / \mathrm{hp} * \mathrm{hr})$ ')

axes (handles. axes5)

plot (handles. coastdown_v_fts, handles . coastdown_lbf)

title ('Road Load (lbf) vs Vehicle Speed (ft/s)')

guidata (hObject, handles)

axes (handles . axes6)

plot (handles. coastdown_laps, handles. slope_force_lbf)

title ('Road Load due to Track')

\%hObject. Data $(1,1)=$ mat 2 cell (handles.engine_rpm $(1), 1)$;

set (handles. max_speed, 'String', handles . vmax);

set (handles.min_speed_tag, 'String', handles.vmin);

set (handles. weight_tag, 'String', handles.m_vehicle $*$ handles.g) ;

set (handles.gear_tag,,'String', handles.gear_ratio);

set (handles.radius_tag, 'String', handles.rwheel);

set (handles.drivetrain_eff_tag, 'String', handles.drivetrain_eff $* 100)$;

set (handles.road_load_poly_edit, 'String', handles.

road_load_poly_Frr)

guidata (hObject, handles) \%this saves the handle data

end

$\%$ UIWAIT makes Design_GUI wait for user response (see UIRESUME)

$\%$ uiwait (handles. figure 1 );

$\%$ - Outputs from this function are returned to the command line.

function varargout $=$ Design_GUI_OutputFcn(hObject, eventdata, handles )

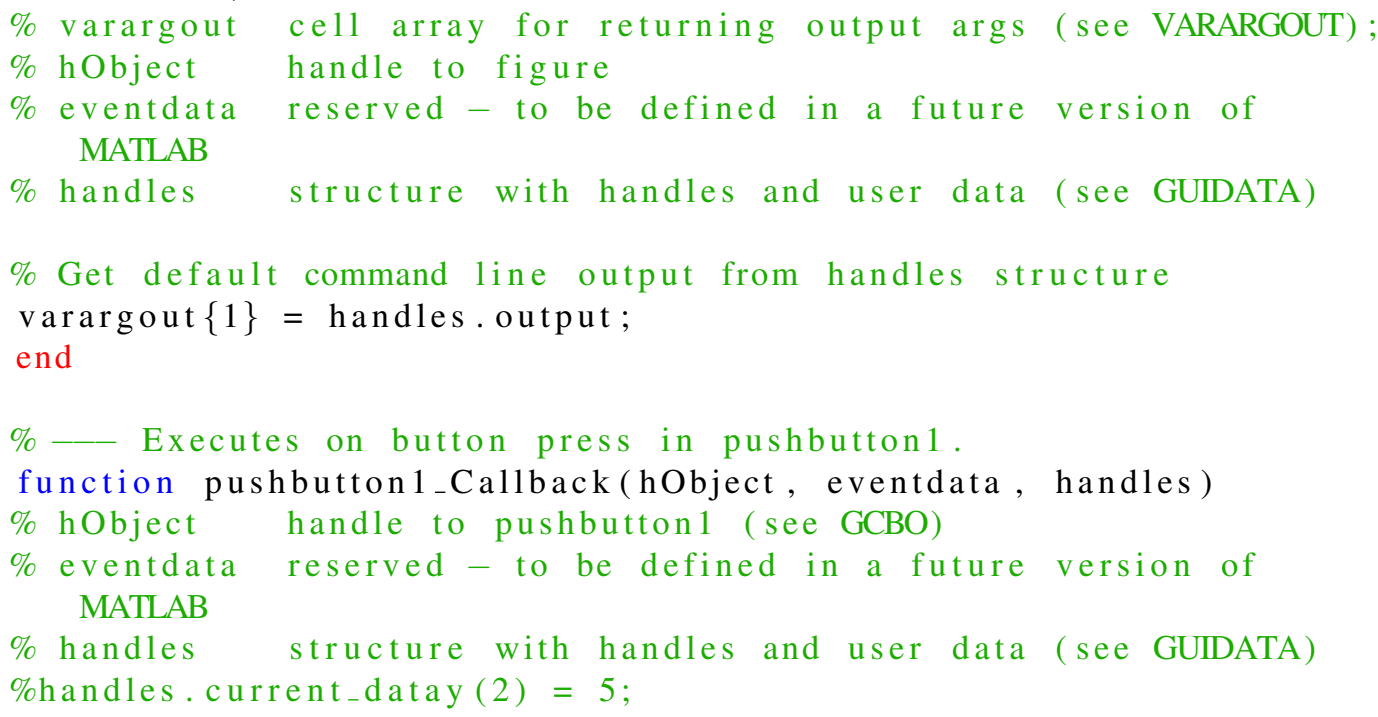




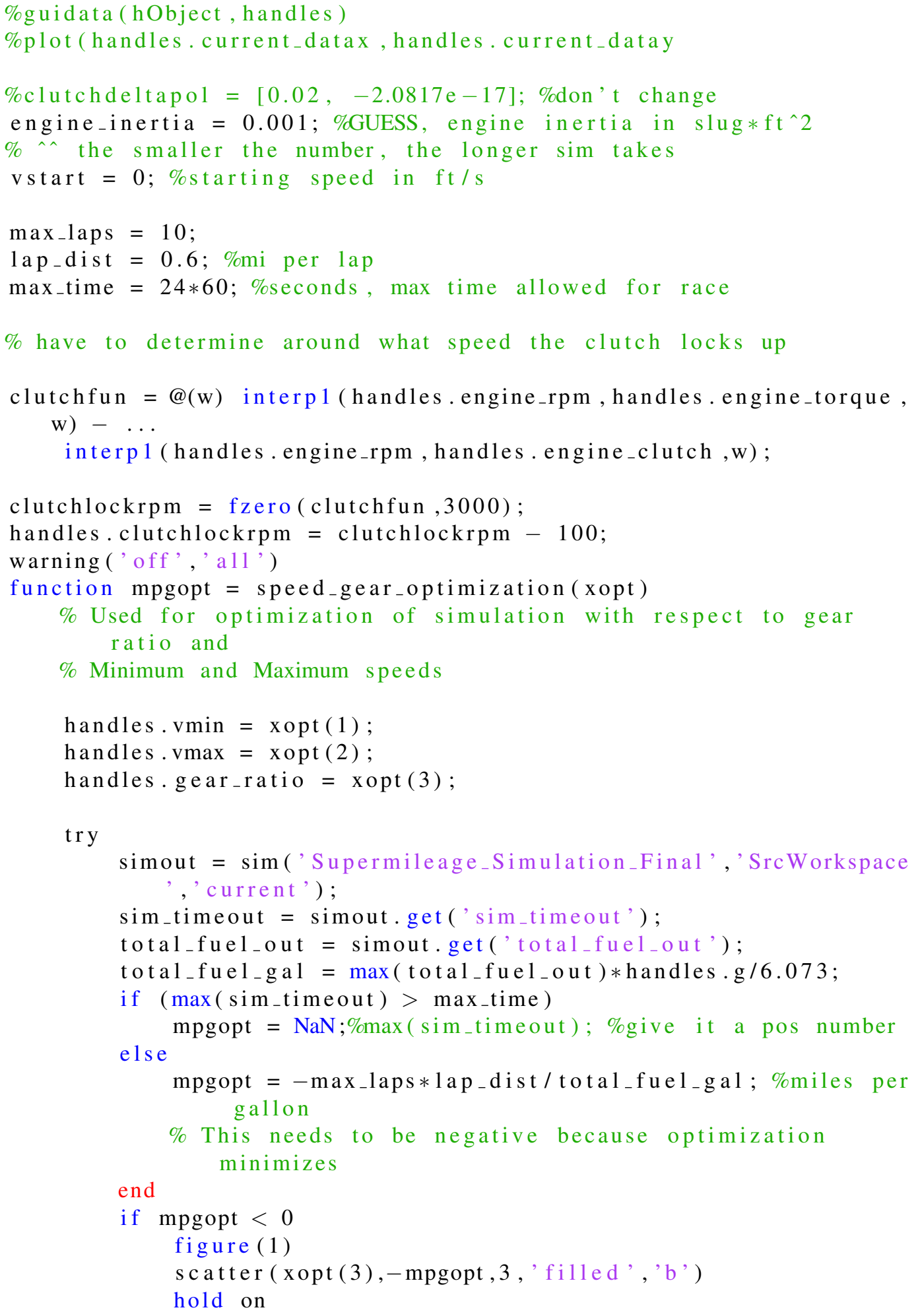




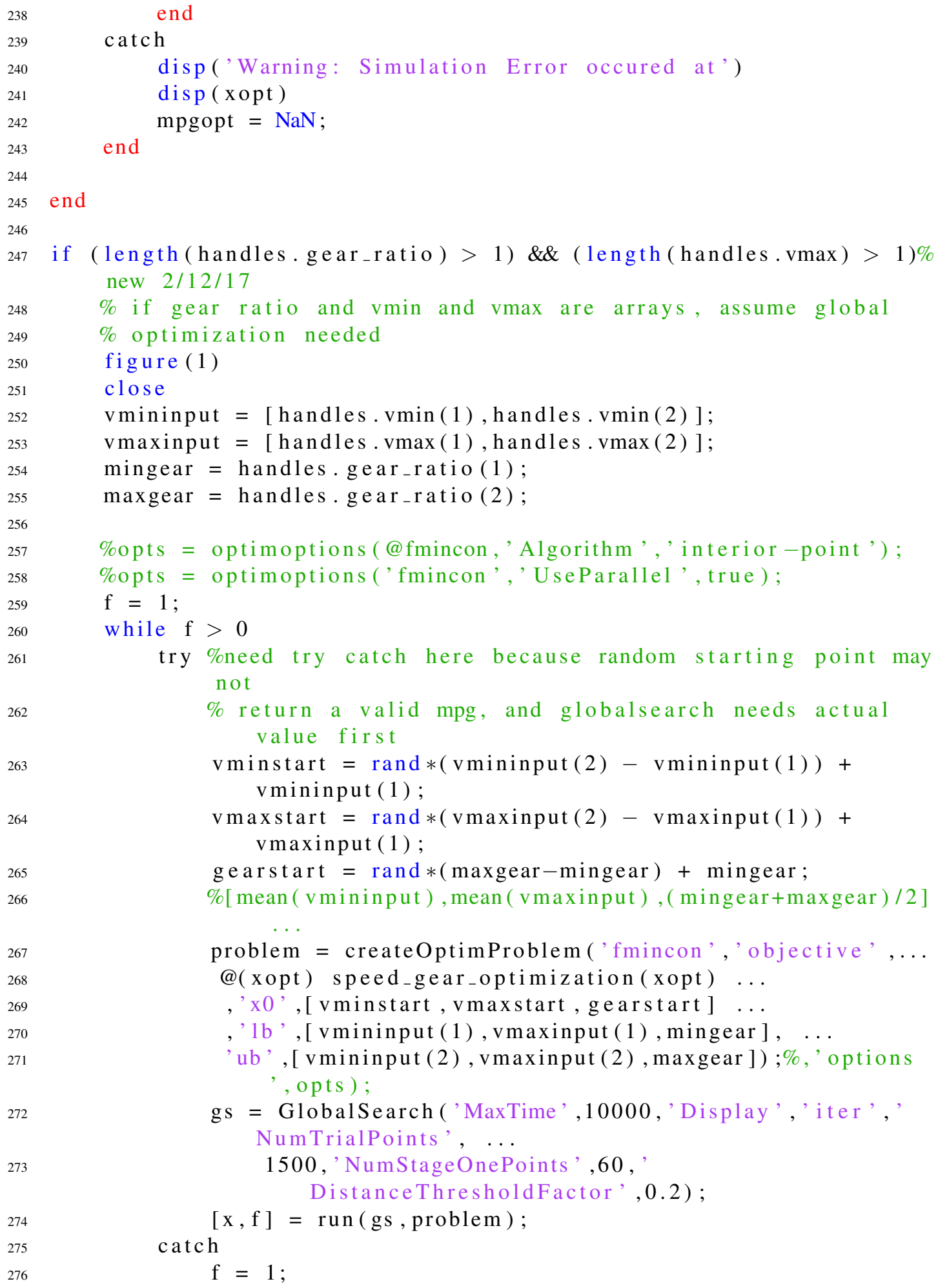




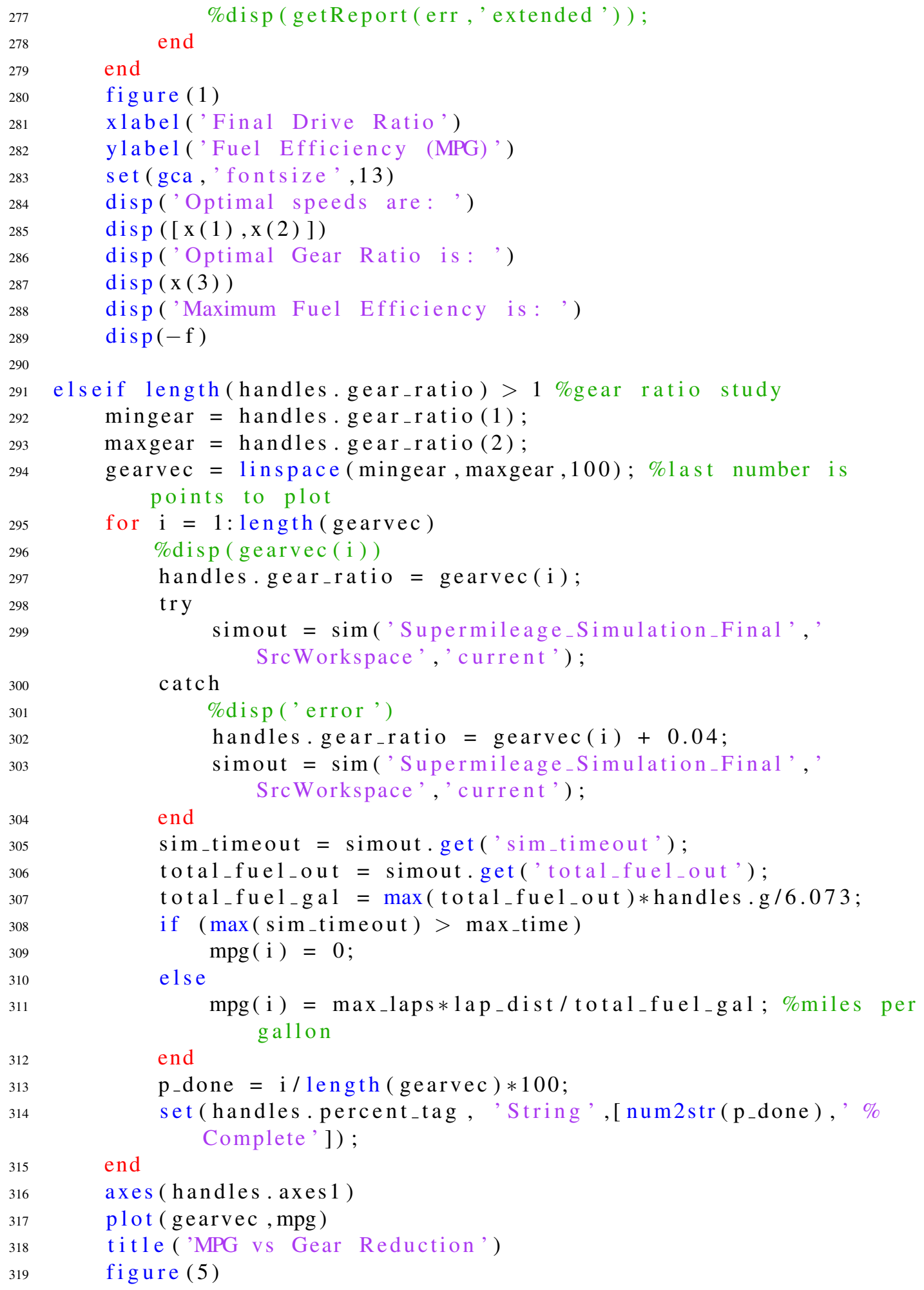




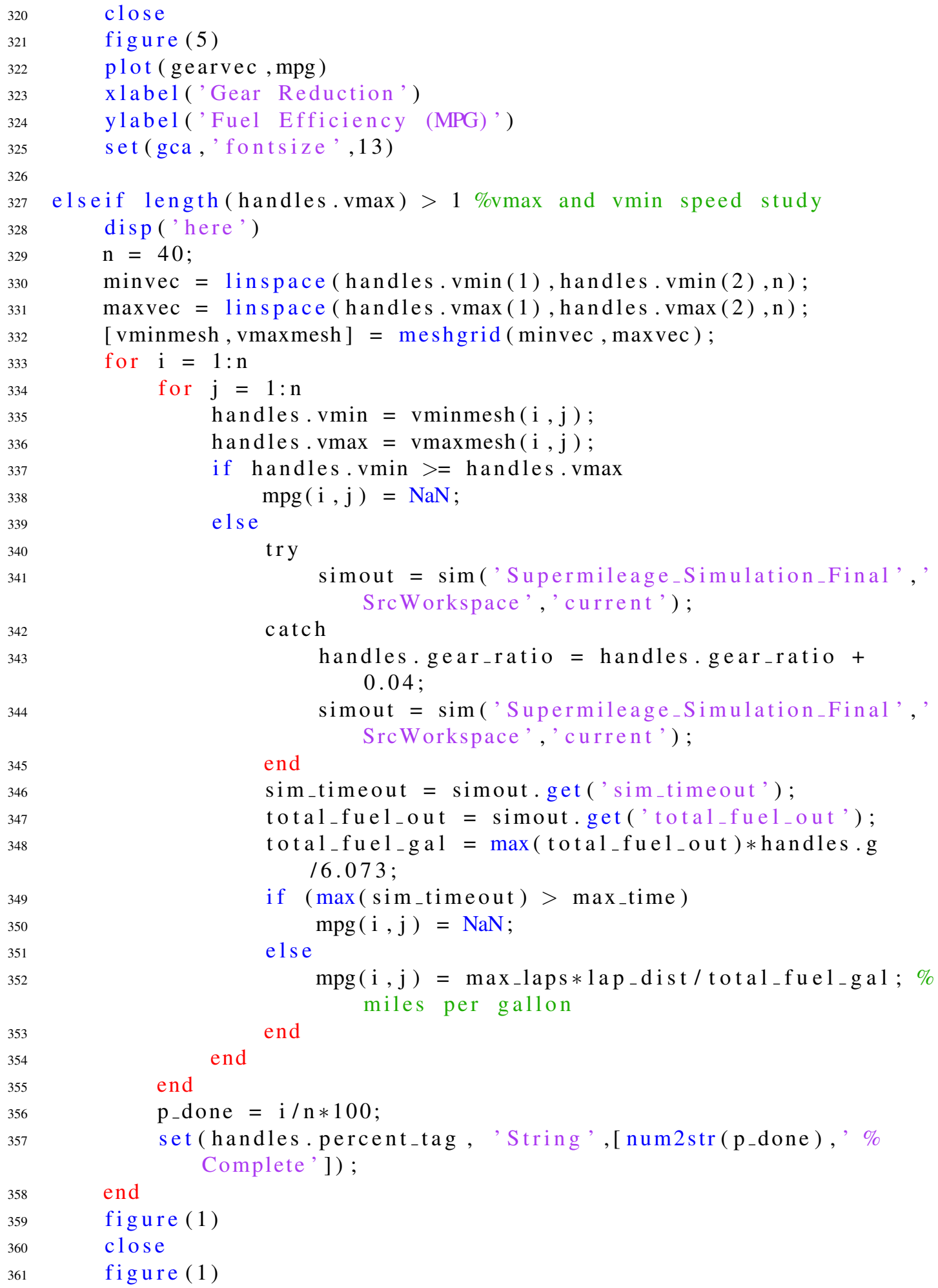




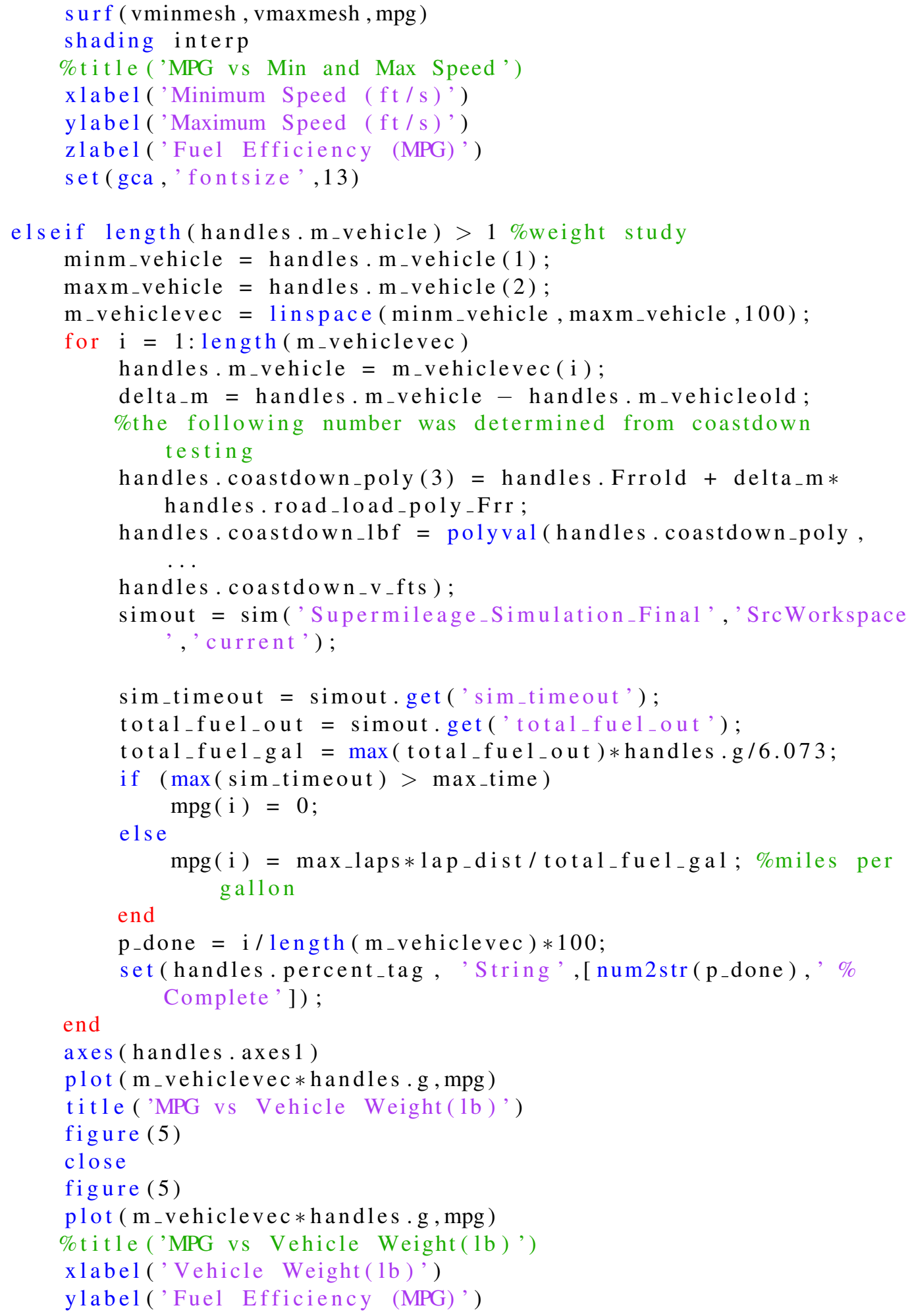


set (gca,' fontsize', 13 )

else \%if everything has only one input value, do a normal 


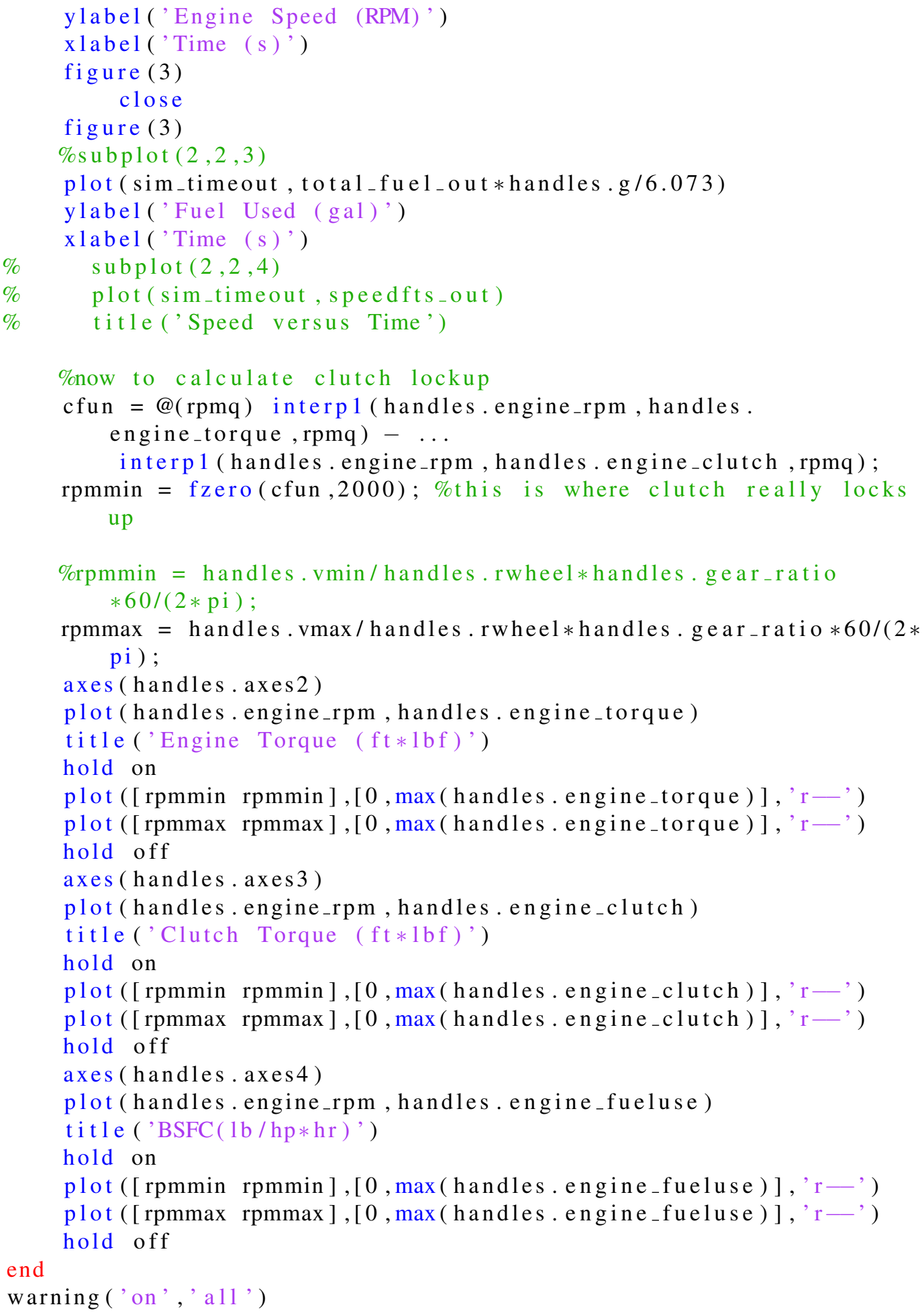




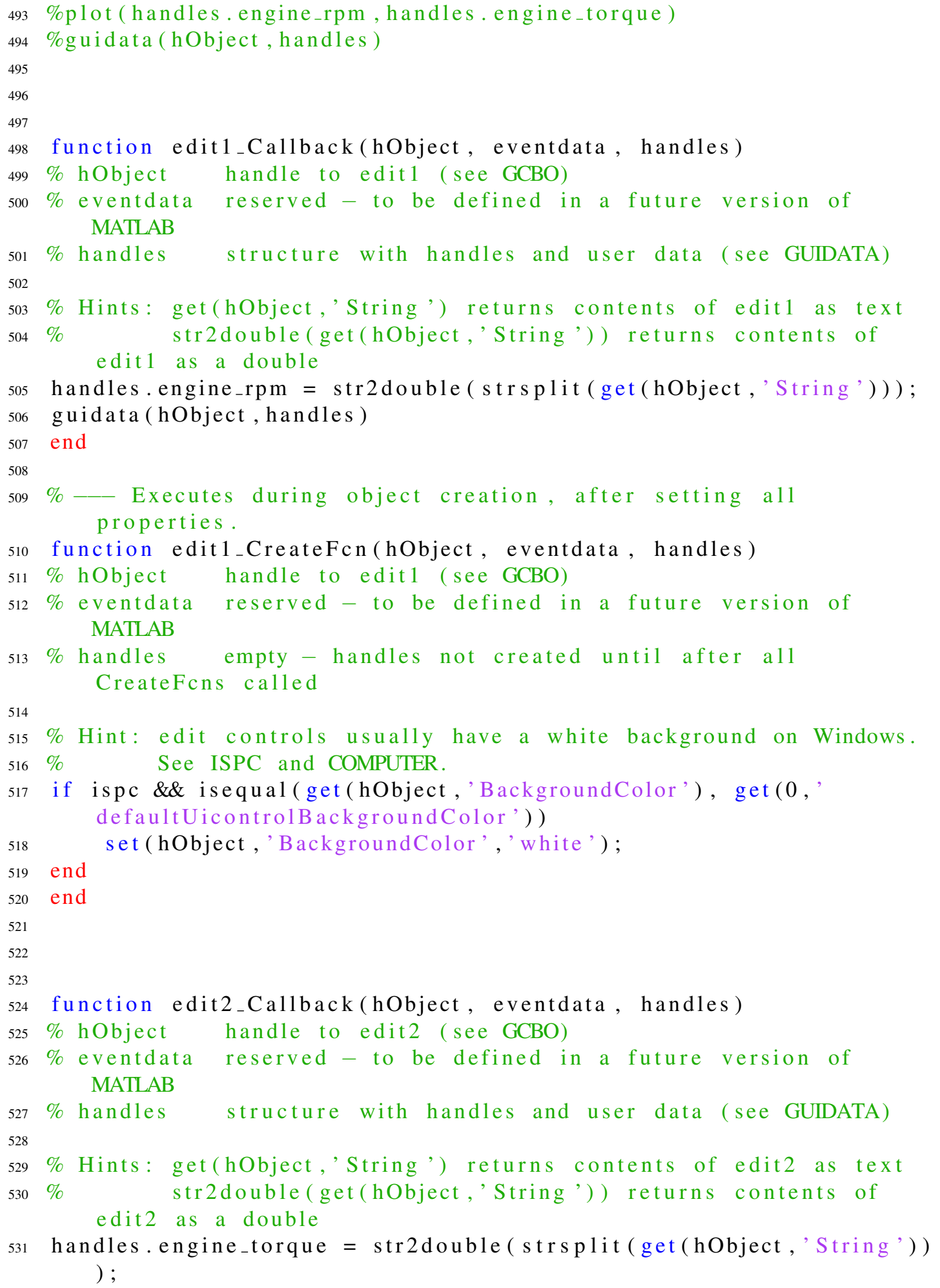




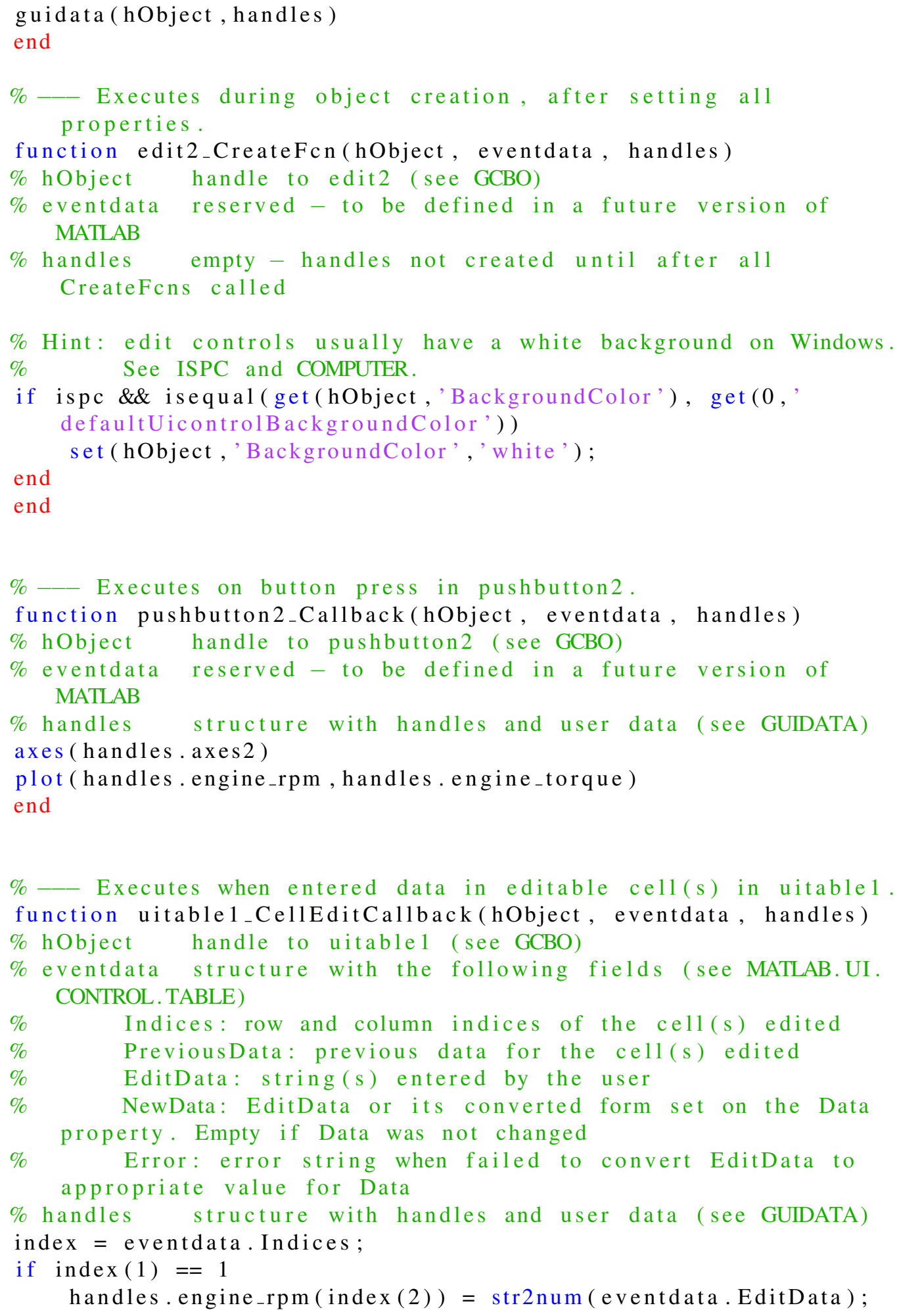




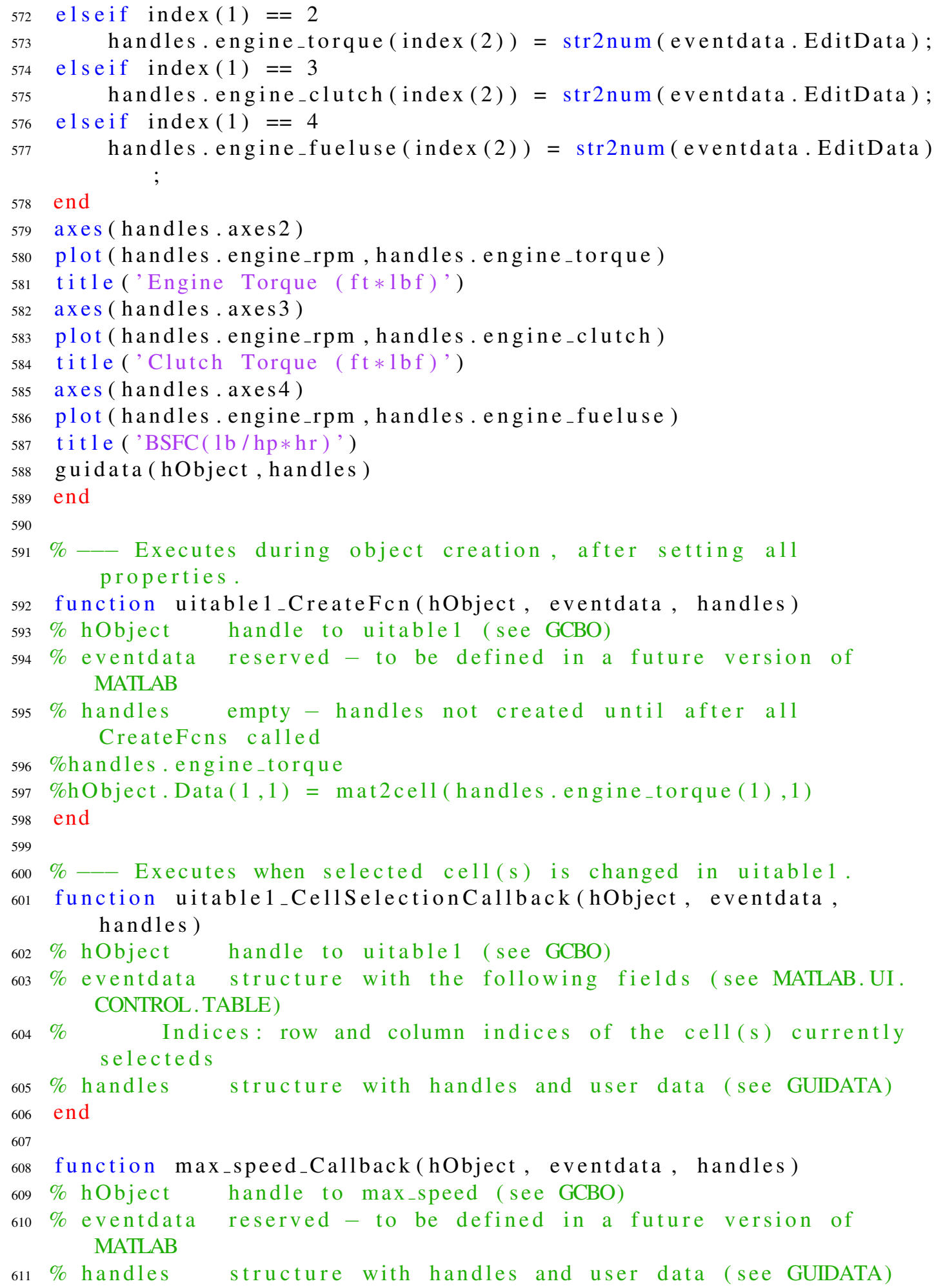


612

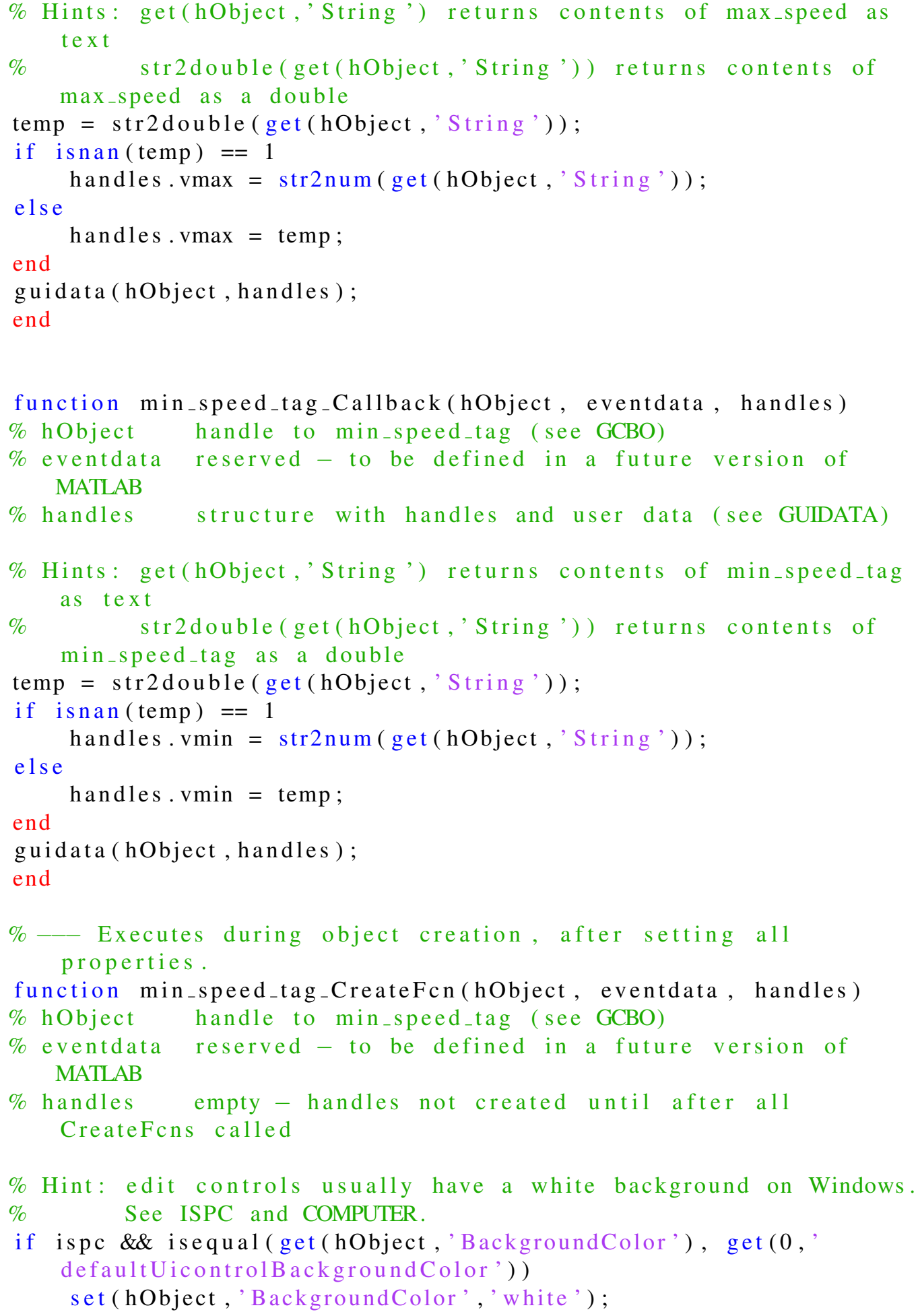


end

end

function weight_tag_Callback(hObject, eventdata, handles)

$\%$ hObject handle to weight_tag (see GCBO)

$\%$ eventdata reserved - to be defined in a future version of MATLAB

\% handles structure with handles and user data (see GUIDATA)

\% Hints: get(hObject, 'String') returns contents of weight_tag as

text

\% str2double(get(hObject, 'String')) returns contents of weight_tag as a double

temp = str2double (get (hObject,'String') $)$;

if isnan(temp) $==1 \%$ there have been 2 weight values input for design study

handles.m_vehicle $=$ str2num( get (hObject, 'String') $) /$ handles.g;

else \%there is only one weight value input

handles.m_vehicle $=$ temp/handles.g;

delta_m $=$ handles.m_vehicle - handles.m_vehicleold;

\%the following number was determined from coastdown testing handles.coastdown_poly $(3)=$ handles.Frrold + delta_m $*$ handles. road_load_poly_Frr;

set (handles.road_load_p, 'String', num2str(handles.

coastdown_poly));

handles. coastdown_lbf = polyval (handles. coastdown_poly, $\ldots$ handles. coastdown_ $\left.\mathrm{v}_{-} \mathrm{fts}\right)$;

axes (handles.axes5)

plot (handles. coastdown_v_fts, handles. coastdown_lbf)

title ('Road Load (1bf) vs Vehicle Speed (ft/s)')

end

guidata (hObject, handles);

end

$\%$ - Executes during object creation, after setting all properties.

function weight_tag_CreateFcn(hObject, eventdata, handles)

$\%$ hObject handle to weight_tag (see GCBO)

$\%$ eventdata reserved - to be defined in a future version of MATLAB

\% handles empty - handles not created until after all

CreateFcns called

\% Hint: edit controls usually have a white background on Windows.

$\% \quad$ See ISPC and COMPUTER.

if ispc \&\& isequal(get(hObject,' BackgroundColor'), get (0, defaultUicontrolBackgroundColor') ) 


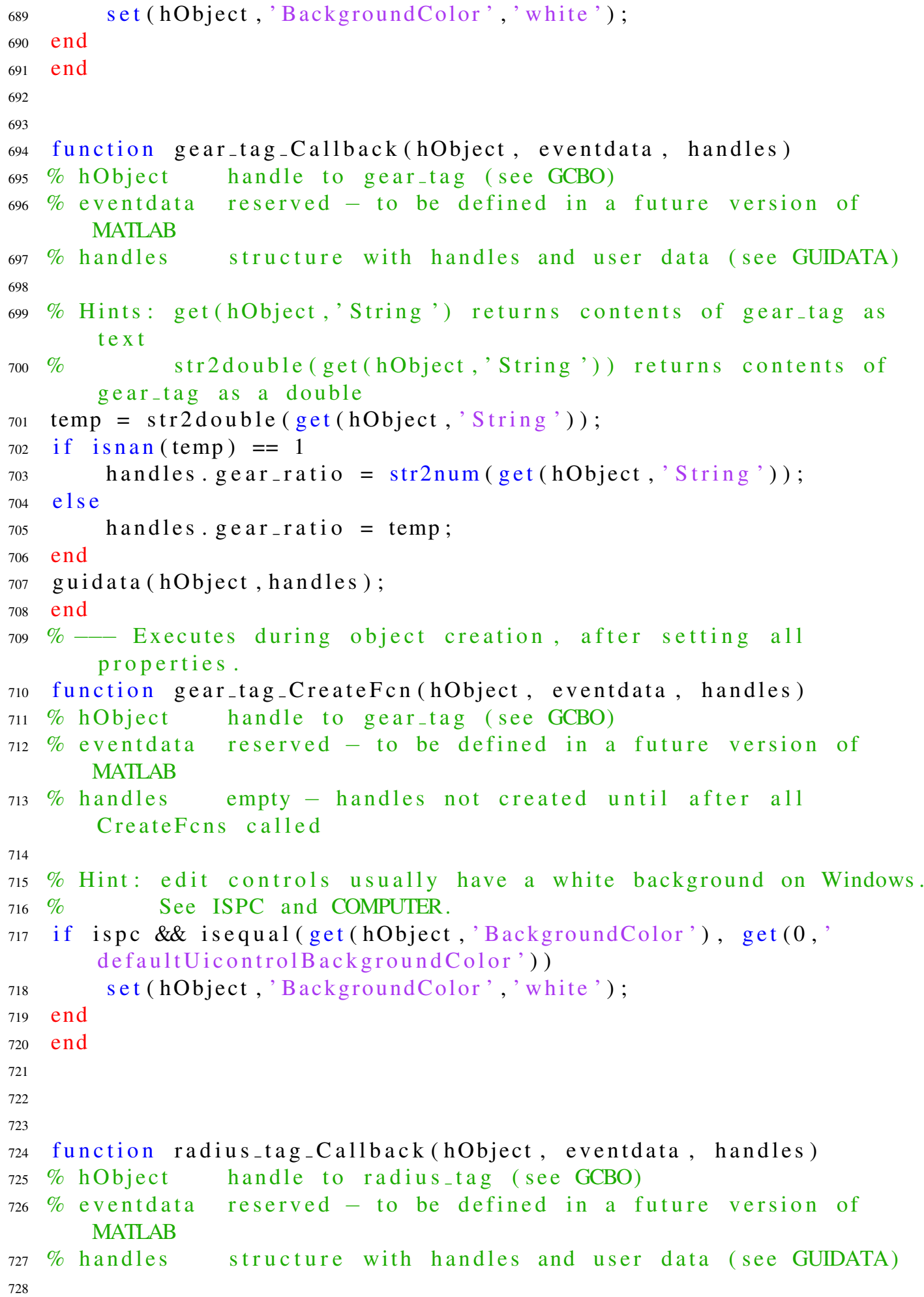




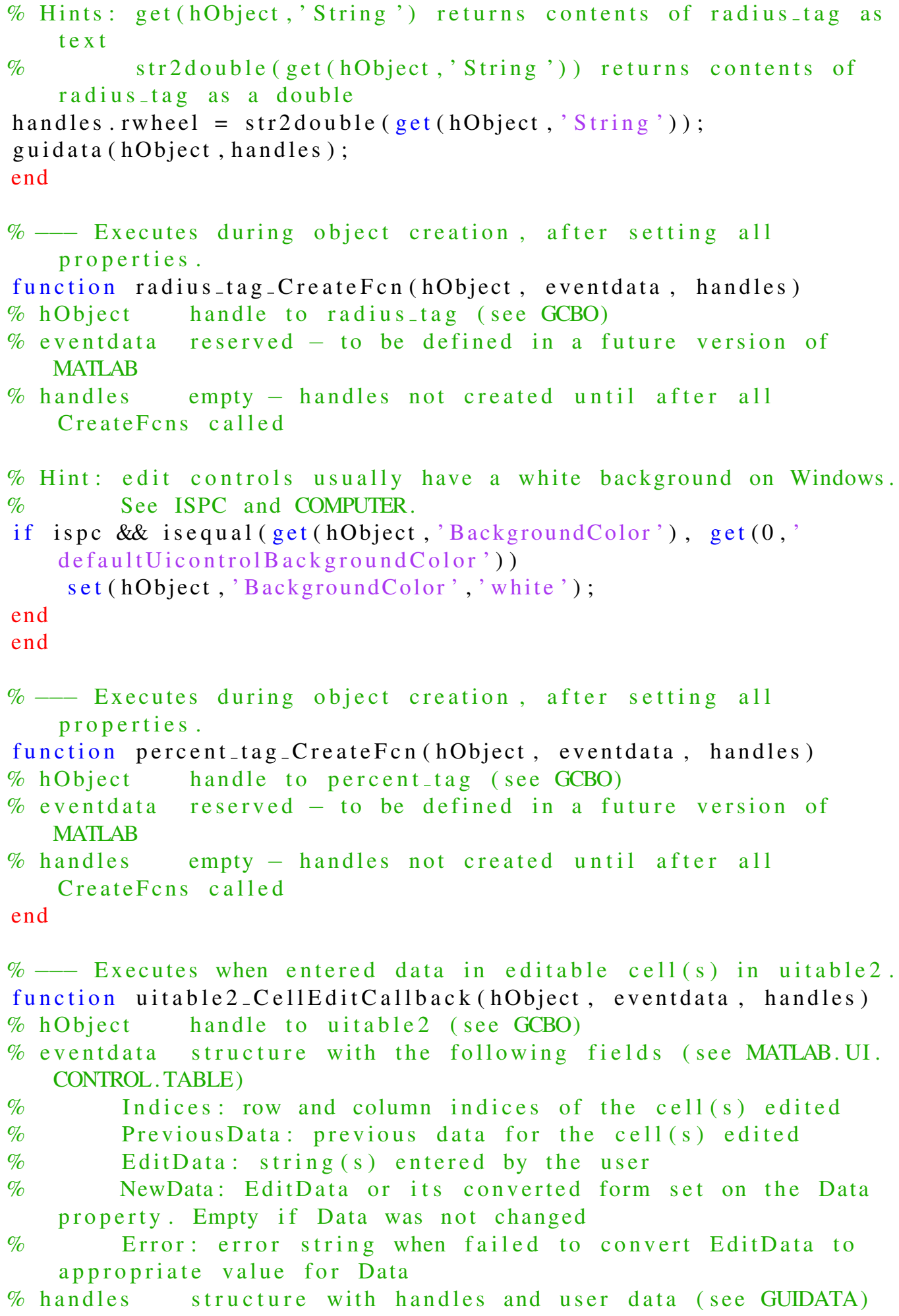


index $=$ eventdata. Indices;

if index (1) == 1 handles. coastdown_v_fts (index (2)) = str2num(eventdata. EditData ) ; 
822

function drivetrain_eff_tag_Callback(hObject, eventdata, handles)

\% hObject handle to drivetrain_eff_tag (see GCBO)

\% eventdata reserved - to be defined in a future version of MATLAB

\% handles structure with handles and user data (see GUIDATA)

\% Hints: get(hObject,' String') returns contents of drivetrain_eff_tag as text

\% str2double(get(hObject,' String')) returns contents of drivetrain_eff_tag as a double

handles.drivetrain_eff $=$ str2num(get(hObject,'String')) $/ 100$;

guidata (hObject, handles)

end

$\%$ - Executes during object creation, after setting all properties.

function drivetrain_eff_tag_CreateFcn (hObject, eventdata, handles)

$\%$ hobject handle to drivetrain_eff_tag (see GCBO)

$\%$ eventdata reserved - to be defined in a future version of MATLAB

\% handles empty - handles not created until after all

CreateFcns called

\% Hint: edit controls usually have a white background on Windows.

$\% \quad$ See ISPC and COMPUTER.

if ispc \&\& isequal(get(hObject, 'BackgroundColor'), get ( 0 ,' defaultUicontrolBackgroundColor') )

end

set (hObject, 'BackgroundColor', 'white');

end

$\%$ Executes when entered data in editable cell(s) in

lap_load_tag.

function lap_load_tag_CellEditCallback(hObject, eventdata, handles )

\% hObject handle to lap_load_tag (see GCBO)

$\%$ eventdata structure with the following fields (see MATLAB. UI. CONTROL. TABLE)

$\% \quad$ Indices: row and column indices of the cell(s) edited

PreviousData: previous data for the cell(s) edited

EditData: string (s) entered by the user

NewData: EditData or its converted form set on the Data property. Empty if Data was not changed

\% Error: error string when failed to convert EditData to appropriate value for Data

\% handles structure with handles and user data (see GUIDATA) index = eventdata. Indices; 
865

866

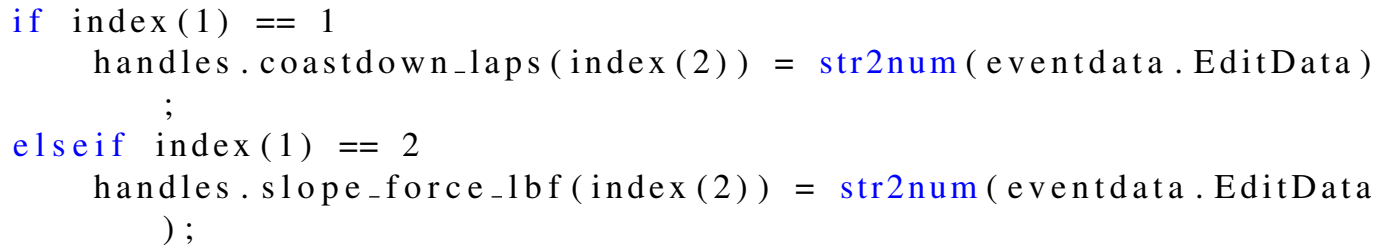




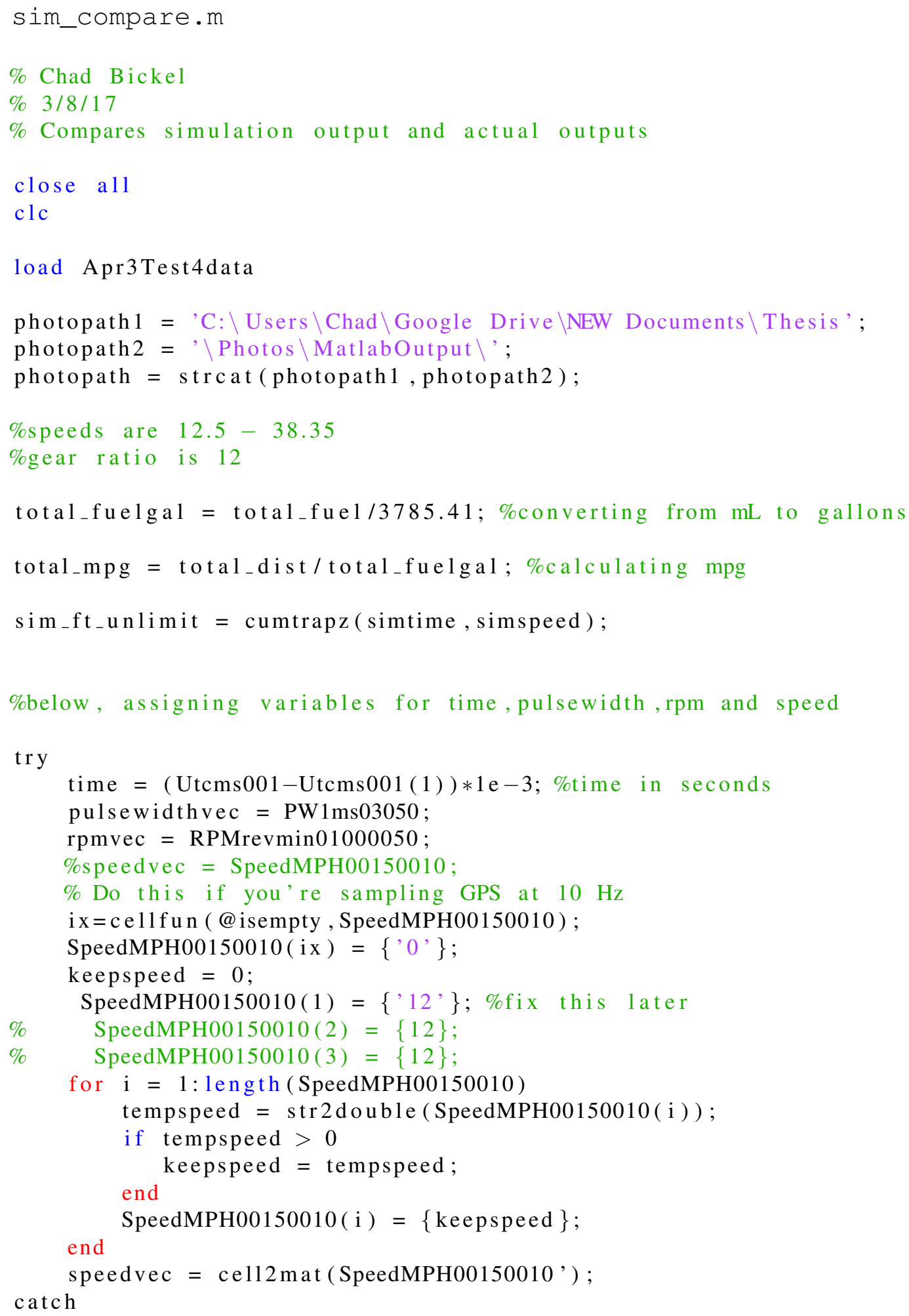




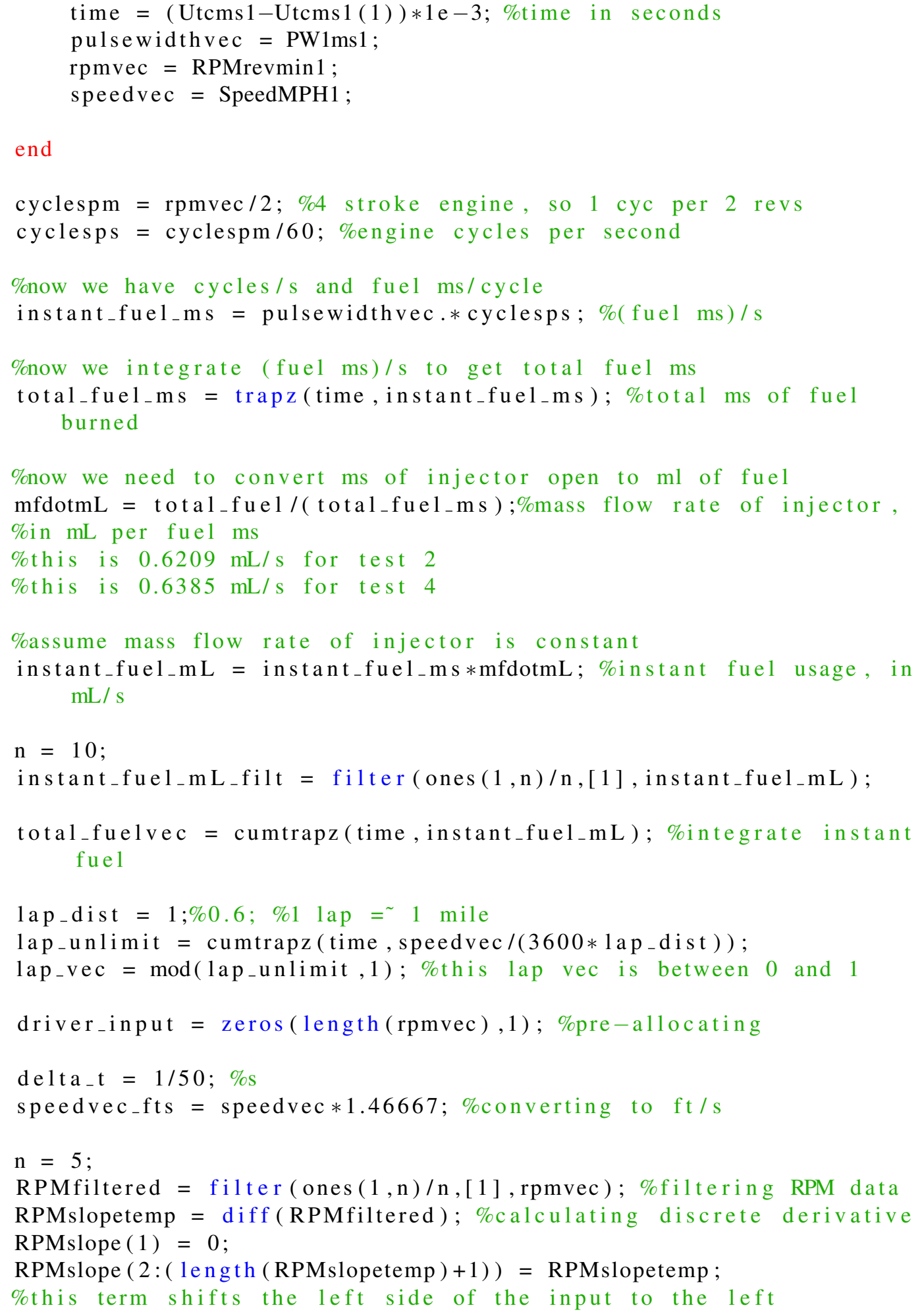




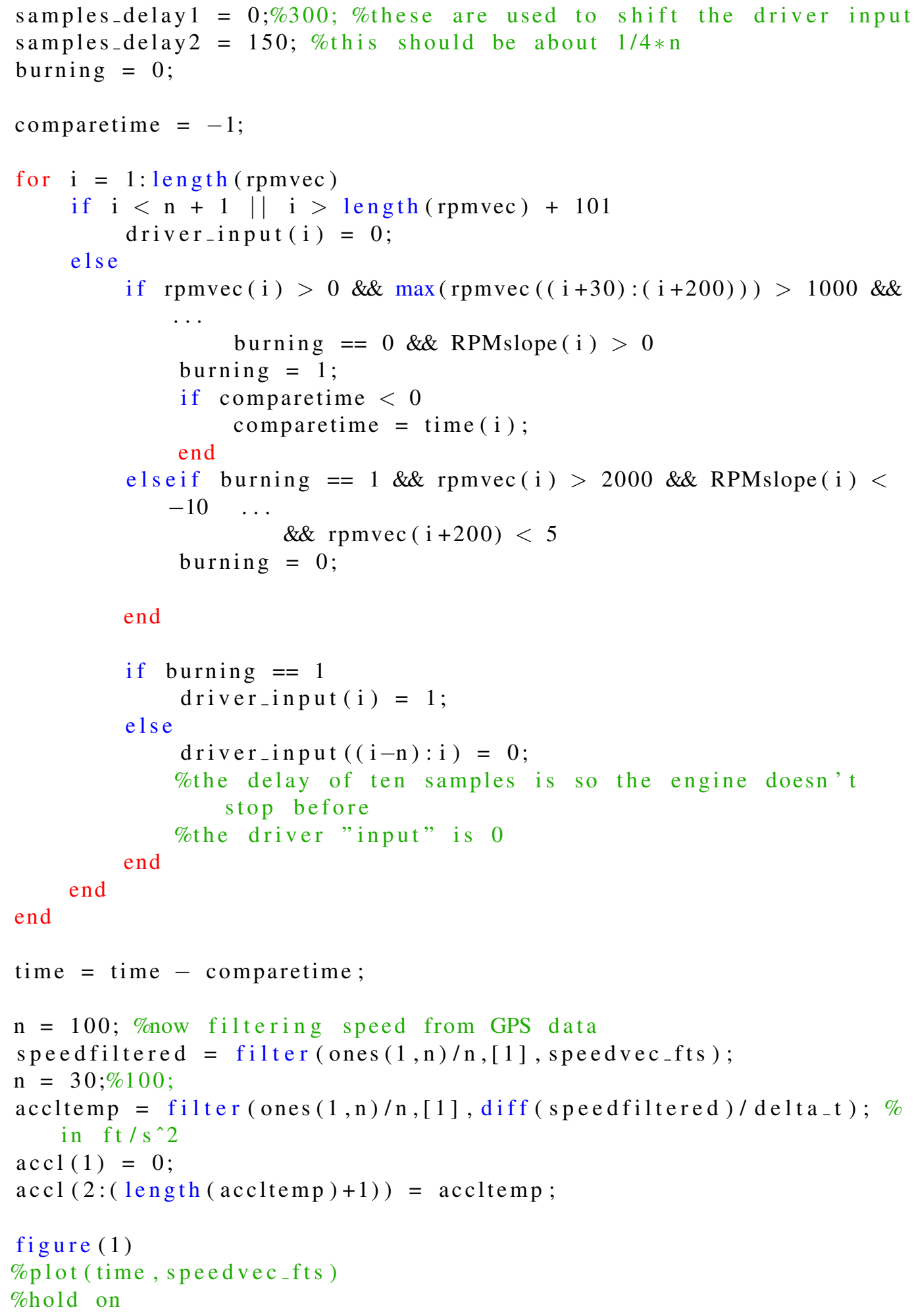




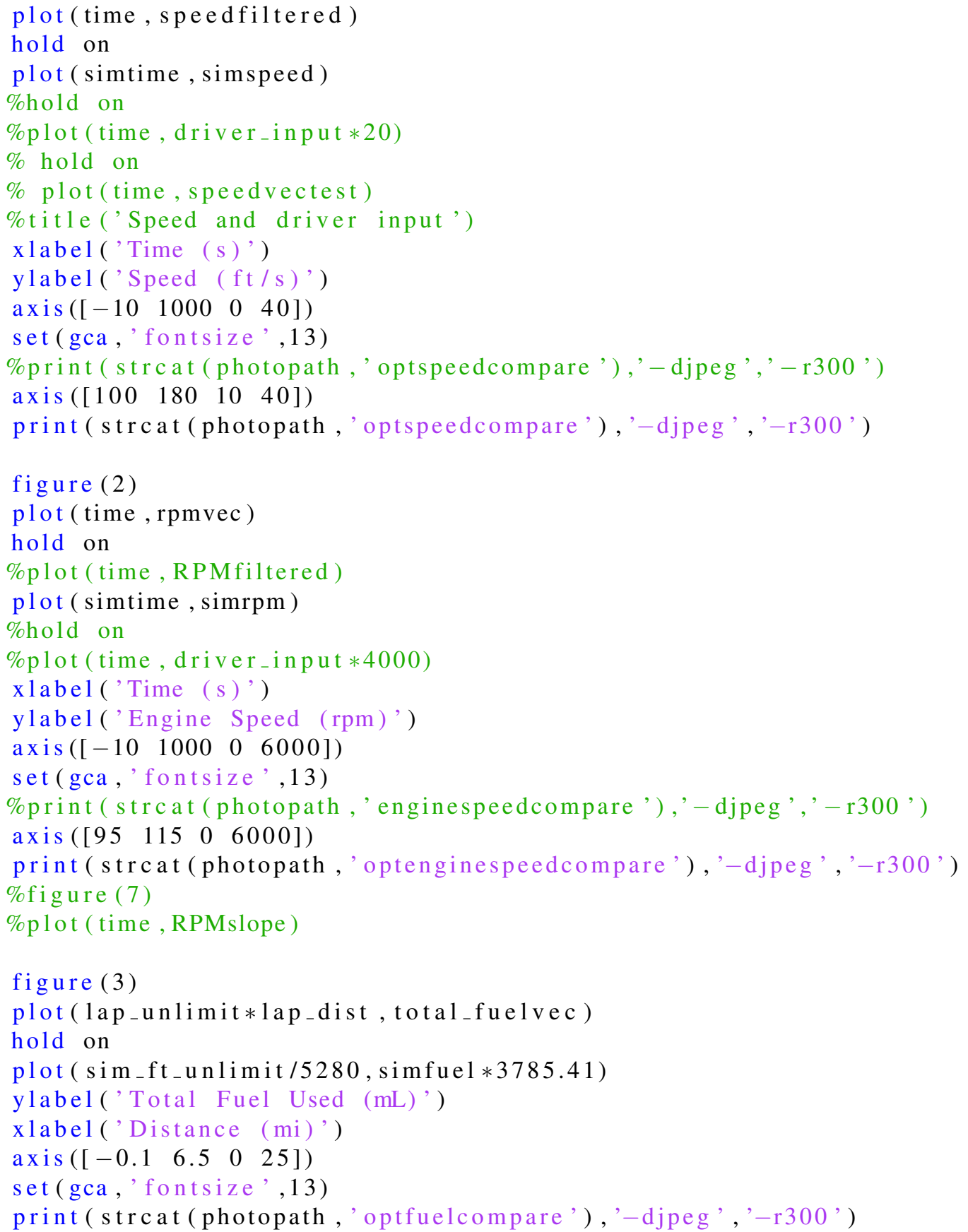

